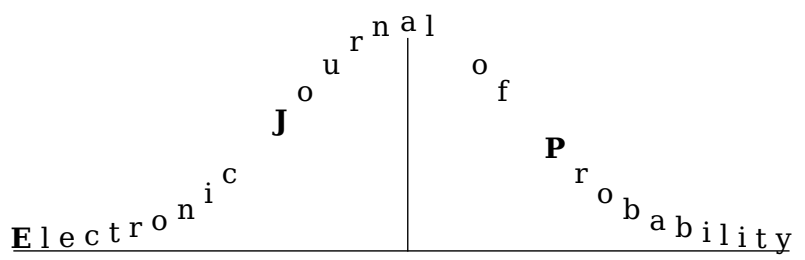

Electron. J. Probab. 26 (2021), article no. 49, 1-41.

ISSN: 1083-6489 https://doi.org/10.1214/21-EJP620

\title{
Completely random measures and Lévy bases in free probability
}

\author{
Francesca Collet* $\quad$ Fabrizio Leisen $^{\dagger} \quad$ Steen Thorbjørnsen $^{\ddagger}$
}

\begin{abstract}
This paper develops a theory for completely random measures in the framework of free probability. A general existence result for free completely random measures is established, and in analogy to the classical work of Kingman it is proved that such random measures can be decomposed into the sum of a purely atomic part and a (freely) infinitely divisible part. The latter part (termed a free Lévy basis) is studied in detail in terms of the free Lévy-Khintchine representation and a theory parallel to the classical work of Rajput and Rosiński is developed. Finally a Lévy-Itô type decomposition for general free Lévy bases is established.
\end{abstract}

Keywords: free completely random measure; free infinite divisibility; free Lévy basis; Lévy-Itô type decomposition.

MSC2020 subject classifications: Primary 46L54, Secondary 60E07; 60G57.

Submitted to EJP on July 10, 2020, final version accepted on March 29, 2021.

Supersedes arXiv:2007.05336v1.

\section{Introduction}

In the paper [11] J.F.C. Kingman introduced the concept of completely random measures. Specifically a random measure on a measurable space $(X, \mathcal{S})$ is a collection $N=\{N(B, \cdot) \mid B \in \mathcal{S}\}$ of non-negative random variables, defined on some probability space $(\Omega, \mathcal{F}, P)$, such that the mapping $B \mapsto N(B, \omega)$ is a measure on the $\sigma$-algebra $\mathcal{S}$ for each fixed $\omega$ in $\Omega$. If the random variables $N\left(B_{1}, \cdot\right), \ldots, N\left(B_{n}, \cdot\right)$ are further assumed to be independent, whenever $B_{1}, \ldots, B_{n}$ are disjoint sets from $\mathcal{S}$, then $N$ is referred to as a completely random measure. Kingman established (under certain additional conditions) that a completely random measure $N$ can always be decomposed into a sum $N_{a}+N_{c}$

\footnotetext{
*Department of Mathematics, University of Padova, via Trieste 63, 35121 Padova, Italy. The author is member of the Gruppo Nazionale per l'Analisi Matematica, la Probabilità e le loro Applicazioni (GNAMPA) of the Istituto Nazionale di Alta Matematica (INdAM). E-mail: francesca.collet@unipd.it

${ }^{\dagger}$ School of Mathematical Sciences, University of Nottingham, University Park, Nottingham NG7 2RD, United Kingdom. E-mail: fabrizio. leisen@gmail.com

${ }^{\ddagger}$ Department of Mathematics, University of Aarhus, Ny Munkegade 118, 8000 Aarhus C, Denmark. Email: steenth@imf .au.dk
} 
of two mutually independent completely random measures, where, for each $\omega, N_{a}(\cdot, \omega)$ is purely atomic, while $N_{c}(\cdot, \omega)$ is atom less. For the second term Kingman showed further that the distribution of the random variable $N_{c}(B, \cdot)$ is infinitely divisible for any $B$ in $\mathcal{S}$, and hence $N_{c}$ is an example of what is nowadays commonly referred to as a Lévy basis. The infinite divisibility of the "marginals" allows for the employment of Lévy-Khintchine techniques, and the resulting theory was developed by B.S. Rajput and J. Rosiński in the celebrated paper [16], where more general "index sets" than $\sigma$-algebras were also considered. To be precise, a Lévy basis ${ }^{1}$ on a ring $\mathcal{E}$ of subsets of $X$ is a family $N=\{N(E, \cdot) \mid E \in \mathcal{E}\}$ of real valued random variables, defined on some probability space $(\Omega, \mathcal{F}, P)$, such that

- For all $E$ in $\mathcal{E}$ the distribution of $N(E, \cdot)$ is an infinitely divisible probability measure on $\mathbb{R}$.

- If $n \in \mathbb{N}$ and $E_{1}, E_{2}, \ldots E_{n}$ are disjoint sets from $\mathcal{E}$, then $N\left(E_{1}, \cdot\right), N\left(E_{2}, \cdot\right), \ldots, N\left(E_{n}, \cdot\right)$ are independent random variables.

- If $\left(E_{n}\right)_{n \in \mathbb{N}}$ is a sequence of disjoint sets from $\mathcal{E}$, such that $\bigcup_{n \in \mathbb{N}} E_{n} \in \mathcal{E}$, then it holds with probability 1 that $N\left(\bigcup_{n \in \mathbb{N}} E_{n}, \cdot\right)=\sum_{n=1}^{\infty} N\left(E_{n}, \cdot\right)$.

In recent years much of the theory of stochastic processes (with very general index sets) has found a subsuming and unifying framework in Lévy bases, and from that perspective it is a natural step in the development of free probability to manifest a corresponding theory for free Lévy bases and more generally free completely random measures. This theory can also be expected to provide a concrete model for the asymptotics of high dimensional random-matrix valued random measures (and integrals with respect to such), which have received some attention recently in various special cases (see e.g. [14], [8] and [13]), but the theory remains to be fully developed.

In this paper we introduce natural counterparts of completely random measures and Lévy bases in the context of free probability, where the classical notion of independence is replaced by that of free independence, and infinite divisibility refers to the corresponding notion of free convolution (see [21] or [3] for an introduction to free probability). We establish thus general existence results for free completely random measures and for free Lévy bases. In addition we prove, in full analogy with the mentioned results of Kingman, that a non-negative free completely random measure $M$ can be decomposed into a sum

$$
M=M_{a}+M_{c}
$$

of two freely independent terms, where $M_{a}$ is purely atomic (in a natural sense) free completely random measure, while $M_{c}$ is a free Lévy basis (thus with freely infinitely divisible marginals). We derive a similar decomposition in the more general situation where the assumption of positivity of the marginals of $M$ is dropped, although some moment conditions need to be imposed in this case. We focus subsequently on free Lévy bases, where the free infinite divisibility of the marginals allows for invoking the Bercovici-Pata bijection (see Subsection 2.4) and thus for transferring major parts of the theory of Rajput and Rosiński to the free setting. The resulting theory subsumes and unifies a major part of the existing theory on free Lévy processes and related topics. Moreover, it includes a theory of integration of deterministic functions with respect to a free Lévy basis, which is further used to establish a Lévy-Itô type decomposition of a general free Lévy basis into the sum of two freely independent terms, the first of which is of free Brownian motion type, while the second is of pure jump type. This result covers in particular the free analog of the result by Pedersen (see [15]) for classical Lévy bases,

\footnotetext{
${ }^{1}$ In [16] a Lévy basis was referred to as an infinitely divisible, independently scattered random measure.
} 
Completely random measures and Lévy bases in free probability

and in another direction it generalizes the Lévy-Itô type decomposition obtained for free Lévy processes in [2]. Inserting the Lévy-Itô decomposition of $M_{c}$ in (1.1) evidently leads to a refined decomposition for general free completely random measures.

The remaining part of this paper is organized as follows. In Section 2 we provide background material on $\delta$-rings (and measures thereon), the measure topology and free infinite divisibility. In Section 3 we give the formal definition of free completely random measures and of free Lévy bases, and we state the mentioned general existence result (the proof of which is deferred to Section 7). We establish furthermore the described analogs of Kingman's decomposition theorem for completely random measures. In Section 4 we develop free analogs of essential parts of the Rajput-Rosiński theory, and in Section 5 we develop a theory of integration with respect to free Lévy bases. In particular we construct free Lévy bases with a deterministic "density" with respect to another (given) free Lévy basis, and this is further used in Section 6, where we establish the described Lévy-Itô type decomposition for free Lévy bases. In the final Section 7 we prove the general existence of free completely random measures and of free Lévy bases. While the existence of "classical" random measures and Lévy bases is generally based on the Kolmogorov extension theorem, our construction is based on free products of von Neumann algebras and the theory of (unbounded) operators affiliated with such. As this construction heavily builds on the theory of operator algebras and is less probabilistic in nature, we have deferred it to the final section of the paper. This is mainly to bring focus to the probabilistic aspects of the developed theory and to emphasize the analogies to the theories of Kingman and of Rajput and Rosiński. Accordingly the first six sections of the paper can be read without reference to the detailed construction given in Section 7 . To our knowledge this construction, dealing throughout with unbounded operators, has not been carried out in detail previously in the literature even for the case of free Lévy processes. The paper concludes with an appendix that covers some specific aspects of the theory of von Neumann algebras needed for the construction in Section 7.

\section{Preliminaries}

In this section we provide background material on various definitions and results that are fundamental for the rest of the paper.

\subsection{Measures on $\delta$-rings}

Recall that a ring of subsets of a non-empty set $X$ is a collection $\mathcal{E}$ of subsets of $X$ satisfying that $A \cup B, A \backslash B \in \mathcal{E}$, whenever $A, B \in \mathcal{E}$. Since $A \cap B=A \backslash(A \backslash B)$, a ring is automatically closed under finite intersections. If $\mathcal{E}$ is even closed under all countable intersections, then it is referred to as a $\delta$-ring. By $\sigma(\mathcal{E})$ we denote the smallest $\sigma$-algebra on $X$ containing $\mathcal{E}$. It is noteworthy that if $\mathcal{E}$ is a $\delta$-ring, then the following implication holds for all subsets $A, E$ of $X$ :

$$
A \in \sigma(\mathcal{E}) \text { and } E \in \mathcal{E} \quad \Longrightarrow \quad A \cap E \in \mathcal{E} .
$$

A (finite) signed measure on a $\delta$-ring $\mathcal{E}$ is a mapping $\Theta: \mathcal{E} \rightarrow \mathbb{R}$ satisfying the following two conditions:

(a) $\Theta(A \cup B)=\Theta(A)+\Theta(B)$ for any disjoint sets $A, B$ from $\mathcal{E}$,

(b) $\lim _{n \rightarrow \infty} \Theta\left(B_{n}\right)=0$ for any decreasing sequence $\left(B_{n}\right)_{n \in \mathbb{N}}$ of sets from $\mathcal{E}$, such that $\bigcap_{n \in \mathbb{N}} B_{n}=\emptyset$.

For sequences $\left(B_{n}\right)_{n \in \mathbb{N}}$ of sets as described in (b), we use the notation $B_{n} \downarrow \emptyset$. Conditions (a) and (b) (together) are equivalent to the condition that $\Theta\left(\bigcup_{n \in \mathbb{N}} E_{n}\right)=$ 
$\sum_{n=1}^{\infty} \Theta\left(E_{n}\right)$ for any sequence $\left(E_{n}\right)_{n \in \mathbb{N}}$ of disjoint sets from $\mathcal{E}$, such that $\bigcup_{n \in \mathbb{N}} E_{n} \in \mathcal{E}$. For a mapping $\Theta: \mathcal{E} \rightarrow[0, \infty]$ this latter condition, together with the condition $\Theta(\emptyset)=0$, defines a (positive) measure on $\mathcal{E}$. In case $\Theta(A) \in[0, \infty)$ for all $A$ in $\mathcal{E}$, we refer to $\Theta$ as a finite measure on $\mathcal{E}$. By a suitable variant of the Carathéodory Extension Theorem, a (positive) measure on $\mathcal{E}$ can always be extended to a (positive) measure on $\sigma(\mathcal{E})$. Note however that the extension of a finite measure may fail to be finite. Correspondingly it does not generally hold that a signed measure on $\mathcal{E}$ can be extended to a signed measure on $\sigma(\mathcal{E})$. In fact, under the additional assumption:

There exists a sequence $\left(U_{n}\right)_{n \in \mathbb{N}}$ of sets from $\mathcal{E}$, such that $\bigcup_{n \in \mathbb{N}} U_{n}=X$,

any signed measure $\Theta$ on a $\delta$-ring $\mathcal{E}$ can be written uniquely in the form:

$$
\Theta(E)=\Theta^{+}(E)-\Theta^{-}(E), \quad(E \in \mathcal{E}),
$$

where $\Theta^{+}, \Theta^{-}$are two (positive) measures on $\sigma(\varepsilon)$, which are singular in the sense that there exists a set $S$ from $\sigma(\mathcal{E})$, such that $\Theta^{+}\left(S^{c}\right)=\Theta^{-}(S)=0$. These $\Theta^{+}, \Theta^{-}$are not necessarily finite measures on $\sigma(\mathcal{E})$, but $\Theta^{+}(E), \Theta^{-}(E)<\infty$ for all $E$ in $\mathcal{E}$, so in particular $\Theta^{+}, \Theta^{-}$are $\sigma$-finite (cf. (2.2)). The unique decomposition (2.3) further allows us to define the total variation measure $|\Theta|$ of $\Theta$ as

$$
|\Theta|(A)=\Theta^{+}(A)+\Theta^{-}(A), \quad(A \in \sigma(\mathcal{E})) .
$$

In this setup we mention finally, that if $\kappa$ is a $\sigma$-finite (positive) measure on $\sigma(\mathcal{E}$ ), such that $|\Theta| \leq \kappa$, then $\Theta^{+}$and $\Theta^{-}$are both absolutely continuous with respect to $\kappa$ with $\sigma(\varepsilon)$-measurable densities $h^{+}, h^{-}: X \rightarrow \mathbb{R}$, which may be chosen such that $h^{+}(x), h^{-}(x) \in[0,1]$ for all $x$ in $X$. Then if we put $h=h^{+}-h^{-}$, it follows for any set $E$ from $\mathcal{E}$ that

$$
\Theta(E)=\int_{E} h^{+} \mathrm{d} \kappa-\int_{E} h^{-} \mathrm{d} \kappa=\int_{E} h \mathrm{~d} \kappa,
$$

so that $\Theta$ has density $h$ with respect to $\kappa$. Note however that $h$ need not be an element of $\mathcal{L}^{1}(\kappa)$.

\subsection{Free Independence}

Free independence (introduced by Voiculescu) is a notion of independence which in many respects behaves similarly to the classical notion of independence of random variables, and it is possible to develop a probability theory based on this notion in parallel to the classical theory of probability. At the same time free independence cannot be observed among classical random variables (except for trivial cases). The right framework for free independence is that of quantum probability, where the random variables are modelled mathematically as selfadjoint operators affiliated with a $W^{*}$ probability space. A $W^{*}$-probability space is a pair $(\mathcal{M}, \tau)$ consisting of a von Neumann algebra $\mathcal{M}$ acting on a Hilbert space $\mathcal{H}$ equipped with a normal faithful tracial state $\tau: \mathcal{M} \rightarrow \mathbb{C}$ (see [21] for details). A (possibly unbounded) operator $a$ in $\mathcal{H}$ is affiliated with $\mathcal{M}$, if $a u=u a$ for any unitary operator $u$ on $\mathcal{H}$ satisfying that $u b=b u$ for all $b$ in $\mathcal{M}$. If $a$ is selfadjoint, this is equivalent to the condition that $f(a) \in \mathcal{M}$ for any bounded Borel function $f: \mathbb{R} \rightarrow \mathbb{R}$, where $f(a)$ is defined in terms of spectral calculus. In this case the spectral distribution of $a$ is the unique Borel-probability measure $L^{\mathrm{sp}}\{a\}$ on $\mathbb{R}$, satisfying that

$$
\int_{\mathbb{R}} f(t) L^{\mathrm{sp}}\{a\}(\mathrm{d} t)=\tau(f(a))
$$

for any bounded Borel-function $f: \mathbb{R} \rightarrow \mathbb{R}$. Throughout the paper we denote by $\mathcal{B F}(\mathbb{R})$ the algebra of all real-valued Borel functions on $\mathbb{R}$. Furthermore we let $\mathcal{B F}_{b}(\mathbb{R})$ denote the subalgebra of bounded functions from $\mathcal{B F}(\mathbb{R})$. 
If $a_{1}, \ldots, a_{n}$ are (possibly unbounded) selfadjoint operators affiliated with $\mathcal{M}$, they are said to be freely independent (with respect to $\tau$ ), if

$$
\tau\left(\left[f_{1}\left(a_{i_{1}}\right)-\tau\left(f_{1}\left(a_{i_{1}}\right)\right)\right]\left[f_{2}\left(a_{i_{2}}\right)-\tau\left(f_{2}\left(a_{i_{2}}\right)\right)\right] \cdots\left[f_{m}\left(a_{i_{m}}\right)-\tau\left(f_{m}\left(a_{i_{m}}\right)\right)\right]\right)=0,
$$

for any $m \in \mathbb{N}$, any functions $f_{1}, \ldots, f_{m}$ from $\mathcal{B F}_{b}(\mathbb{R})$ and any $i_{1}, \ldots, i_{m}$ from $\{1, \ldots, n\}$, such that $i_{1} \neq i_{2}, i_{2} \neq i_{3}, \ldots, i_{m-1} \neq i_{m}$.

If $\mathcal{A}$ is a unital subalgebra of $\mathcal{M}$, we denote by $\mathcal{A}^{\circ}$ the subspace of centered elements of $\mathcal{A}$, i.e. $\mathcal{A}^{\circ}=\{a \in \mathcal{A} \mid \tau(a)=0\}$. A finite number of unital subalgebras $\mathcal{A}_{1}, \ldots, \mathcal{A}_{n}$ of $\mathcal{M}$ are said to be freely independent, provided that

$$
\tau\left(a_{1} a_{2} \cdots a_{m}\right)=0
$$

whenever $a_{1} \in \mathcal{A}_{i_{1}}^{\circ}, \ldots, a_{m} \in \mathcal{A}_{i_{m}}^{\circ}$ for suitable $i_{1}, \ldots, i_{m} \in\{1, \ldots, n\}$ such that $i_{1} \neq i_{2}$, $i_{2} \neq i_{3}, \ldots, i_{m-1} \neq i_{m}$. For any collection $\left\{T_{i} \mid i \in I\right\}$ of selfadjoint operators affiliated with $\mathcal{M}$ we denote by $\operatorname{Alg}\left(\left\{T_{i} \mid i \in I\right\}\right)$ (respectively $W^{*}\left(\left\{T_{i} \mid i \in I\right\}\right)$ ) the unital subalgebra (respectively $W^{*}$-subalgebra) of $\mathcal{M}$ generated by the subset $\left\{f\left(T_{i}\right) \mid i \in I, f \in \mathcal{B F}_{b}(\mathbb{R})\right\}$ of $\mathcal{M}$. We refer to $\operatorname{Alg}\left(\left\{T_{i} \mid i \in I\right\}\right)$ and $W^{*}\left(\left\{T_{i} \mid i \in I\right\}\right)$ as, respectively, the unital subalgebra and the $W^{*}$-subalgebra of $\mathcal{M}$ generated by $\left\{T_{i} \mid i \in I\right\}$. It follows then that selfadjoint operators $a_{1}, \ldots, a_{n}$ affiliated with $\mathcal{M}$ are freely independent, exactly when they generate freely independent unital subalgebras (or, equivalently, $W^{*}$-subalgebras) of $\mathcal{M}$.

\subsection{The measure topology}

For a $W^{*}$-probability space $(\mathcal{M}, \tau)$ we denote by $\overline{\mathcal{M}}$ the space of closed, densely defined (possibly unbounded) operators affiliated with $\mathcal{M}$. Then $\overline{\mathcal{M}}$ is a $*$-algebra under the adjoint operation and the so called strong sum and strong product. For example the strong sum of two operators $a, a^{\prime}$ from $\overline{\mathcal{M}}$ is the closure of the operator $a+a^{\prime}$, and the strong product is defined similarly.

For any positive numbers $\epsilon, \delta$ we introduce the subset $N(\epsilon, \delta)$ of $\overline{\mathcal{M}}$ given by

$$
N(\epsilon, \delta)=\left\{a \in \overline{\mathcal{M}} \mid \tau\left[1_{(\epsilon, \infty)}(|a|)\right]<\delta\right\},
$$

where $|a|=\left(a^{*} a\right)^{1 / 2}$. The measure topology on $\overline{\mathcal{M}}$ is the vector space topology on $\overline{\mathcal{M}}$ for which the sets $N(\epsilon, \delta), \epsilon, \delta \in(0, \infty)$, form a neighborhood basis at 0 . In this topology the adjoint operation and the (strong-) sum and product are all continuous operations. In addition the measure topology satisfies the first axiom of countability and is a complete Hausdorff topology.

For a sequence $a, a_{1}, a_{2}, a_{3}, \ldots$ of operators from $\overline{\mathcal{M}}$ we have that

$$
a_{n} \rightarrow a \quad \text { in the measure topology } \quad \Longleftrightarrow \quad L^{\mathrm{sp}}\left\{\left|a_{n}-a\right|\right\} \stackrel{\mathrm{W}}{\rightarrow} \delta_{0} \quad \text { as } n \rightarrow \infty,
$$

and thus convergence in the measure topology is the quantum probability analog of convergence in probability. For that reason, and for brevity, we will occasionally use the notation $a_{n} \stackrel{\mathrm{P}}{\rightarrow} a$ or $a=\mathrm{P}-\lim _{n \rightarrow \infty} a_{n}$ to express that a sequence $\left(a_{n}\right)_{n \in \mathbb{N}}$ converges to $a$ in the measure topology.

If $a, a_{1}, a_{2}, a_{3}, \ldots$ are all selfadjoint, we note further the implications:

$$
\begin{aligned}
a_{n} \rightarrow a \text { in the measure topology } & \Longleftrightarrow L^{\mathrm{sp}}\left\{a_{n}-a\right\} \stackrel{\mathrm{w}}{\rightarrow} \delta_{0} \quad \text { as } n \rightarrow \infty \\
& \Longrightarrow L^{\mathrm{sp}}\left\{a_{n}\right\} \stackrel{\mathrm{w}}{\rightarrow} L^{\mathrm{sp}}\{a\} .
\end{aligned}
$$

For more details about the measure topology (and some proofs) we refer to the appendix of [3]. A more complete account on this topic can be found in [18]. 


\subsection{Free infinite divisibility and the free cumulant transform}

If $a$ and $b$ are two freely independent selfadjoint operators affiliated with $\mathcal{M}$, then the free convolution $L^{\mathrm{sp}}\{a\} \boxplus L^{\mathrm{sp}}\{b\}$ of their spectral distributions is defined as the spectral distribution of the sum $a+b$. Since $L^{\mathrm{sp}}\{a+b\}$ is uniquely determined by $L^{\mathrm{sp}}\{a\}, L^{\mathrm{sp}}\{b\}$ and the condition (2.4), and since any pair $(\mu, \nu)$ of Borel-probability measures on $\mathbb{R}$ may be realized as the spectral distributions of two freely independent selfadjoint operators affiliated with some $W^{*}$-probability space, the operation $\boxplus$ is a well-defined binary operation on the class $\mathcal{P}(\mathbb{R})$ of all (Borel-) probability measures on $\mathbb{R}$ (see [7] for details). The corresponding class of infinitely divisible probability laws is denoted by $\mathcal{J D}(\boxplus)$. Thus a measure $\nu$ from $\mathcal{P}(\mathbb{R})$ belongs to $\mathcal{J} \mathcal{D}(\boxplus)$, if and only if

$$
\forall n \in \mathbb{N} \exists \nu_{1 / n} \in \mathcal{P}(\mathbb{R}): \nu=\nu_{1 / n} \boxplus \nu_{1 / n} \boxplus \cdots \boxplus \nu_{1 / n} \quad(n \text { terms }) .
$$

The class of infinitely divisible probability laws with respect to classical convolution $*$ of probability measures is correspondingly denoted by $\mathcal{J D}(*)$.

As in classical probability, free infinite divisibility is generally studied through a Lévy-Khintchine type representation of the free analog of the Fourier transform; the so called free cumulant transform. Specifically the free cumulant transform of a measure $\nu$ from $\mathcal{J D}(\boxplus)$ is defined by the formula:

$$
\mathcal{C}_{\nu}(z)=z G_{\nu}^{\langle-1\rangle}(z)-1
$$

where $G_{\nu}^{\langle-1\rangle}$ denotes the inverse of the Cauchy transform $G_{\nu}$ given by

$$
G_{\nu}(z)=\int_{\mathbb{R}} \frac{1}{z-x} \nu(\mathrm{d} x), \quad\left(z \in \mathbb{C}^{+}\right) .
$$

This inverse (and hence $\mathcal{C}_{\nu}$ ) is always well-defined in a region (depending on $\nu$ ) of the lower half complex plane $\mathbb{C}^{-}$in the form:

$$
\mathcal{D}_{\nu}=\left\{z \in \mathbb{C}^{-}|| z \mid<\delta \text { and } \operatorname{Arg}(z) \in\left(-\frac{\pi}{2}-\epsilon,-\frac{\pi}{2}+\epsilon\right)\right\} .
$$

For a selfadjoint operator $a$ affiliated with a $W^{*}$-probability space $(\mathcal{M}, \tau)$ we shall also use the notation $\mathcal{C}_{a}$ for the free cumulant transform $\mathcal{C}_{L^{\text {sp }}\{a\}}$ of the spectral distribution of $a$. The key property of the free cumulant transform is that it linearizes free additive convolution in the sense that

$$
\mathcal{C}_{\nu \boxplus \nu^{\prime}}(z)=\mathcal{C}_{\nu}(z)+\mathcal{C}_{\nu^{\prime}}(z)
$$

for any probability measures $\nu, \nu^{\prime}$ on $\mathbb{R}$.

In case $\nu$ has finite $p$-th moment for some $p$ in $\mathbb{N}, \mathcal{C}_{\nu}$ admits a Taylor expansion centered at 0 in the form:

$$
\mathcal{C}_{\nu}(z)=\sum_{j=1}^{p} \kappa_{j}(\nu) z^{j}+o\left(z^{p}\right),
$$

where the coefficients $\kappa_{1}(\nu), \ldots, \kappa_{p}(\nu)$ are the free cumulants of $\nu$ (see [5, Theorem 1.3]). These were introduced by Speicher via the moment-cumulant formula:

$$
\kappa_{p}(\nu)=\int_{\mathbb{R}} t^{p} \nu(\mathrm{d} t)-\sum_{\pi \in \mathrm{NC}^{\prime}(p)} \prod_{V \in \operatorname{BL}(\pi)} \kappa_{\# V}(\nu),
$$

from which the free cumulants are defined recursively. In (2.8) $\mathrm{NC}^{\prime}(p)$ is the set of non-crossings partitions of $\{1, \ldots, p\}$ with at least two blocks. For such a partition $\pi$, $\operatorname{BL}(\pi)$ denotes the family of blocks of $\pi$, while, for $V$ in $\operatorname{BL}(\pi)$, \#V denotes the cardinality 
of the corresponding subset of $\{1, \ldots, p\}$ (see [12, Chapter 2] for details). In accordance with (2.6) and (2.7) the free cumulants linearize free additive convolution in the sense that

$$
\kappa_{j}\left(\nu \boxplus \nu^{\prime}\right)=\kappa_{j}(\nu)+\kappa_{j}\left(\nu^{\prime}\right), \quad(j=1, \ldots, p),
$$

whenever $\nu, \nu^{\prime}$ both have finite $p$-th moment.

A measure $\nu$ from $\mathcal{P}(\mathbb{R})$ is in $\mathcal{J D}(\boxplus)$, if and only if $\mathcal{C}_{\nu}$ has the free Lévy-Khintchine representation:

$$
\mathcal{C}_{\nu}(z)=a z+b z^{2}+\int_{\mathbb{R}}\left(\frac{1}{1-t z}-1-z \varsigma(t)\right) r(\mathrm{~d} t), \quad\left(z \in \mathbb{C}^{-}\right),
$$

where $a \in \mathbb{R}, b \in[0, \infty), r$ is a Lévy measure on $\mathbb{R}$ and $\varsigma$ is the function given by ${ }^{2}$

$$
\varsigma(t)=-1_{(-\infty, 1)}(t)+t 1_{[-1,1]}(t)+1_{(1, \infty)}(t), \quad(t \in \mathbb{R}) .
$$

The triplet $(a, b, r)$ is uniquely determined and is referred to as the free characteristic triplet of $\nu$. Recall in comparison that a measure $\mu$ from $\mathcal{P}(\mathbb{R})$ belongs to $\mathcal{J D}(*)$ if and only if its Fourier transform $\hat{\mu}$ has the Lévy-Khintchine representation:

$$
\hat{\mu}(y)=\exp \left(\mathrm{i} a y-\frac{1}{2} b t^{2}+\int_{\mathbb{R}}\left(\mathrm{e}^{\mathrm{i} t y}-1-\mathrm{i} y \varsigma(t)\right) r(\mathrm{~d} t)\right), \quad(y \in \mathbb{R}),
$$

where the parameters $(a, b, r)$ are exactly as above, uniquely determined by $\mu$ and referred to as the (classical) characteristic triplet of $\mu$.

From the two Lévy-Khintchine representations above, it is apparent that there is a one-to-one correspondence $\Lambda$ from $\mathcal{J D}(*)$ onto $\mathcal{J D}(\boxplus)$. Specifically $\Lambda$ maps the probability measure in $\mathcal{I D}(*)$ with classical characteristic triplet $(a, b, r)$ onto the probability measure in $\mathcal{J D}(\boxplus)$ with free characteristic triplet $(a, b, r)$. Although $\Lambda$ may appear as a rather formal correspondence, it has the following fundamental properties for all $\mu_{1}, \mu_{2}$ in $\mathcal{J D}(*)$ and all $c$ in $\mathbb{R}$ :

(i) $\Lambda\left(\mu_{1} * \mu_{2}\right)=\Lambda\left(\mu_{1}\right) \boxplus \Lambda\left(\mu_{2}\right)$,

(ii) $\Lambda\left(D_{c} \mu\right)=D_{c} \Lambda(\mu)$,

(iii) $\Lambda\left(\delta_{c}\right)=\delta_{c}$

(iv) $\Lambda$ is a homeomorphism with respect to weak convergence.

In (ii) and in the following we use the notation $D_{c} \mu$ for the scaling of $\mu$ by the constant $c$, i.e. $D_{c} \mu(B)=\mu\left(c^{-1} B\right)$, if $c \neq 0$, while $D_{0} \mu=\delta_{0}$. Here, as in (iii), $\delta_{c}$ denotes the Dirac measure at $c$.

The probability laws appearing in the free analogs of the Central Limit Theorem and the Poisson Limit Theorem are the semi-circle distributions:

$$
\gamma_{c, \ell}(\mathrm{d} t)=\frac{2}{\pi \ell^{2}} \sqrt{\ell^{2}-(t-c)^{2}} 1_{[c-\ell, c+\ell]}(t) \mathrm{d} t, \quad(\ell>0, c \in \mathbb{R}),
$$

and, respectively, the Marchenko-Pastur distributions

$$
\operatorname{mp}_{\ell}(\mathrm{d} t)= \begin{cases}(1-\ell) \delta_{0}+\frac{1}{2 \pi t} \sqrt{(t-s)(u-t)} 1_{[s, u]}(t) \mathrm{d} t, & \text { if } \ell \in(0,1) \\ \frac{1}{2 \pi t} \sqrt{(t-s)(u-t)} 1_{[s, u]}(t) \mathrm{d} t, & \text { if } \ell \in[1, \infty)\end{cases}
$$

\footnotetext{
2 To emphasize the analogy to the theory developed by Rajput and Rosiński, we have chosen to work throughout with the same centering function $\varsigma$ as the one used in [16], one of the advantages of which is continuity.
} 
where $s=(1-\ell)^{2}$ and $u=(1+\ell)^{2}$. Correspondingly the mapping $\Lambda$ maps Gaussian distributions to semi-circular distributions and Poisson distributions onto MarchenkoPastur distributions. The latter are also referred to as free Poisson distributions.

It will prove important for us to express weak convergence of probability measures in $\mathcal{J D}(\boxplus)$ in terms of the free characteristic triplets in analogy with e.g. the classical result [17, Theorem 8.7].

Theorem 2.1. Let $\nu, \nu_{1}, \nu_{2}, \nu_{3}, \ldots$ be probability measures from $\mathcal{I D}(\boxplus)$ with free characteristic triplets, respectively $(a, b, r),\left(a_{1}, b_{1}, r_{1}\right),\left(a_{2}, b_{2}, r_{2}\right), \ldots$. Then $\nu_{n} \rightarrow \nu$ weakly as $n \rightarrow \infty$, if and only if the following conditions are satisfied:

(i) $a_{n} \rightarrow a$ as $n \rightarrow \infty$.

(ii) $\int_{\mathbb{R}} f(t) r_{n}(\mathrm{~d} t) \rightarrow \int_{\mathbb{R}} f(t) r(\mathrm{~d} t)$ as $n \rightarrow \infty$ for any continuous bounded function $f: \mathbb{R} \rightarrow \mathbb{R}$ vanishing in a neighborhood of 0 .

(iii) $\lim _{\epsilon \downarrow 0}\left(\limsup _{n \rightarrow \infty}\left|b_{n}-b+\int_{[-\epsilon, \epsilon]} t^{2} r_{n}(\mathrm{~d} t)\right|\right)=0$.

Theorem 2.1 follows immediately by combining the corresponding classical result ([17, Theorem 8.7]) with the fact that $\Lambda$ is a homeomorphism.

Another useful characterization of weak convergence for measures in $\mathcal{I D}(\boxplus)$ is the following:

Proposition 2.2. Let $\nu, \nu_{1}, \nu_{2}, \nu_{3}, \ldots$ be probability measures from $\mathcal{J D}(\boxplus)$. Then the following two conditions are equivalent:

(i) $\nu_{n} \stackrel{\mathrm{w}}{\rightarrow} \nu$ as $n \rightarrow \infty$.

(ii) $\lim _{n \rightarrow \infty} \mathcal{C}_{\nu_{n}}$ (i $\left.y\right)=\mathcal{C}_{\nu}(\mathrm{i} y)$ for all $y$ in $(-\infty, 0)$.

For general probability measures (outside $\mathcal{J D}(\boxplus)$ ) the condition in Proposition 2.2 needs to be supplemented by the condition:

$$
\sup _{n \in \mathbb{N}}\left|\mathcal{C}_{\nu_{n}}(\mathrm{i} y)\right| \longrightarrow 0, \quad \text { as } y \uparrow 0,
$$

in order to ensure weak convergence. Proposition 2.2 may be established as a consequence of Theorem 2.1 and the corresponding classical result. We provide here, for the reader's convenience, a short proof which bypasses arithmetics with characteristic triplets.

Proof of Proposition 2.2. As indicated, it is well-known that (i) implies (ii). For the converse implication we recall from [1] that the formula:

$$
\widehat{\Upsilon(\mu)}(y)=\exp \left(\mathcal{C}_{\Lambda(\mu)}(\mathrm{i} y)\right) \quad(\mu \in \mathcal{J D}(*), y \in(-\infty, 0))
$$

defines an injective mapping $\Upsilon: \mathcal{I D}(*) \rightarrow \mathcal{I D}(*)$, which is a homeomorphism onto its range with respect to weak convergence (see [4]). Putting $\mu=\Lambda^{-1}(\nu)$ and $\mu_{n}=\Lambda^{-1}\left(\nu_{n}\right)$, the assumption of the proposition implies that $\lim _{n \rightarrow \infty} \widehat{\Upsilon\left(\mu_{n}\right)}(y)=\widehat{\Upsilon(\mu)}(y)$ for all $y$ in $(-\infty, 0)$, and by complex conjugation this also holds for all positive $y$. Hence by the continuity theorem for Fourier transforms we have that $\Upsilon\left(\mu_{n}\right) \stackrel{\mathrm{w}}{\rightarrow} \Upsilon(\mu)$ as $n \rightarrow \infty$, and since $\Upsilon$ is a homeomorphism, this means that $\mu_{n} \stackrel{\text { w }}{\rightarrow} \mu$ as $n \rightarrow \infty$. Since $\Lambda$ is continuous with respect to weak convergence, this further implies that $\nu_{n} \stackrel{\text { w }}{\rightarrow} \nu$ as $n \rightarrow \infty$, as desired. 


\section{Free completely random measures}

Definition 3.1. Let $X$ be a non-empty set, and let $\mathcal{E}$ be a ring of subsets of $X$. Then a free Lévy basis (FLB) on $(X, \mathcal{E})$ is a family $M=\{M(E) \mid E \in \mathcal{E}\}$ of selfadjoint operators, affiliated with some $W^{*}$-probability space $(\mathcal{M}, \tau)$, satisfying the following four conditions:

(a) If $E_{1}, \ldots, E_{n}$ are disjoint sets from $\mathcal{E}$, then $M\left(E_{1}\right), \ldots, M\left(E_{n}\right)$ are freely independent with respect to $\tau$.

(b) If $E_{1}, \ldots, E_{n}$ are disjoint sets from $\mathcal{E}$, then $M\left(\bigcup_{j=1}^{n} E_{j}\right)=M\left(E_{1}\right)+\cdots+M\left(E_{n}\right)$.

(c) $L^{\mathrm{sp}}\left\{M\left(E_{n}\right)\right\} \stackrel{\mathrm{w}}{\rightarrow} \delta_{0}$ as $n \rightarrow \infty$ for any decreasing sequence of sets $E_{n}$ from $\mathcal{E}$, satisfying that $\bigcap_{n \in \mathbb{N}} E_{n}=\emptyset$.

(d) $L^{\mathrm{sp}}\{M(E)\} \in \mathcal{J D}(\boxplus)$ for all $E$ in $\mathcal{E}$.

If $M$ satisfies only conditions (a)-(b) above, it is referred to as a finitely additive free random measure (FAFRM). If $M$ satisfies conditions (a)-(c) it is termed a free completely random measure (FCRM).

Remarks 3.2. (1) From Definition 3.1 it is immediate to check that if $t \in \mathbb{R}$ and $M^{1}$ and $M^{2}$ are two freely independent free Lévy bases (respectively FAFRMs or FCRMs) then $t M^{1}+M^{2}$ is again a free Lévy basis (respectively FAFRM or FCRM).

(2) In full analogy with the classical theory of random measures we note that if $M=$ $\{M(E) \mid E \in \mathcal{E}\}$ is a FAFRM then the remaining condition (c) in Definition 3.1 in order for $M$ to be a FCRM is equivalent to the requirement that

$$
\sum_{k=1}^{n} M\left(E_{n}\right) \underset{n \rightarrow \infty}{\longrightarrow} M\left(\bigcup_{n \in \mathbb{N}} E_{n}\right) \quad \text { in the measure topology }
$$

for any sequence $\left(E_{n}\right)_{n \in \mathbb{N}}$ of disjoint sets from $\mathcal{E}$, satisfying that $E:=\bigcup_{n \in \mathbb{N}} E_{n} \in \mathcal{E}$. Indeed, for such a sequence $\left(E_{n}\right)_{n \in \mathbb{N}}$ condition (c) entails that

$$
L^{\mathrm{sp}}\left\{M\left(E \backslash\left(\bigcup_{j=1}^{n} E_{j}\right)\right)\right\} \stackrel{\mathrm{w}}{\longrightarrow} \delta_{0}, \quad \text { as } n \rightarrow \infty,
$$

and hence by property (b) we obtain for any positive $\epsilon$ that

$$
\begin{aligned}
\tau\left[1_{\mathbb{R} \backslash[-\epsilon, \epsilon]}\left(M(E)-\sum_{j=1}^{n} M\left(E_{j}\right)\right)\right] & =\tau\left[1_{\mathbb{R} \backslash[-\epsilon, \epsilon]}\left(M\left(E \backslash\left(\bigcup_{j=1}^{n} E_{j}\right)\right)\right)\right] \\
& =\int_{\mathbb{R}} 1_{\mathbb{R} \backslash[-\epsilon, \epsilon]} L^{\operatorname{sp}}\left\{M\left(E \backslash\left(\bigcup_{j=1}^{n} E_{j}\right)\right)\right\}(\mathrm{d} t) \\
& \underset{n \rightarrow \infty}{\longrightarrow} \delta_{0}(\mathbb{R} \backslash[-\epsilon, \epsilon])=0,
\end{aligned}
$$

which means that $\sum_{j=1}^{n} M\left(E_{j}\right) \rightarrow M\left(\bigcup_{n \in \mathbb{N}} E_{n}\right)$ in the measure topology. Similar argumentation yields that condition (3.1) implies condition (c) of Definition 3.1.

Concerning existence of the various types of free random measures we have the following main result:

Theorem 3.3. Let $\mathcal{E}$ be a $\delta$-ring in a non-empty set $X$, and for each $E$ in $\mathcal{E}$ let $\nu(E, \cdot)$ be a Borel probability measure on $\mathbb{R}$. Assume that whenever $E_{1}, \ldots, E_{n}$ are disjoint sets from $\mathcal{E}$ we have that

$$
\nu\left(\bigcup_{j=1}^{n} E_{j}, \cdot\right)=\nu\left(E_{1}, \cdot\right) \boxplus \cdots \boxplus \nu\left(E_{n}, \cdot\right) .
$$

Then the following assertions hold:

(i) There exists a $W^{*}$-probability space $(\mathcal{M}, \tau)$ and a FAFRM $M=\{M(E) \mid E \in \mathcal{E}\}$ affiliated with $(\mathcal{M}, \tau)$, such that $L^{\mathrm{sp}}\{M(E)\}=\nu(E, \cdot)$ for all $E$ in $\mathcal{E}$. 
(ii) If the given family $\{\nu(E, \cdot) \mid E \in \mathcal{E}\}$ satisfies that $\nu\left(E_{n}, \cdot\right) \stackrel{\mathrm{W}}{\rightarrow} \delta_{0}$ as $n \rightarrow \infty$ for any sequence $\left(E_{n}\right)_{n \in \mathbb{N}}$ of sets from $\mathcal{E}$, such that $E_{n} \downarrow \emptyset$, then $M$ described in (i) is automatically a FCRM.

(iii) If the given family $\{\nu(E, \cdot) \mid E \in \mathcal{E}\}$ satisfies, in addition to the condition in (ii), that $\nu\left(E_{n}, \cdot\right) \in \mathcal{J D}(\boxplus)$ for all $E$ in $\mathcal{E}$, then $M$ described in (i) is automatically a FLB.

Note that condition (3.2) entails that $\nu(\emptyset)=\delta_{0}$. The proof of Theorem 3.3 is obtained as the culmination of a series of preliminary lemmas and is deferred to the concluding section of the paper. In the remainder of the present section we focus on establishing, under various additional assumptions, a decomposition of a FCRM into the sum of a FLB and a "purely atomic part", such that the two terms in the decomposition are freely independent. In order to derive these decompositions we shall need the following natural result.

Lemma 3.4. Let $\mathcal{E}$ be a $\delta$-ring in a non-empty set $X$, and let $M=\{M(E) \mid E \in \mathcal{E}\}$ be a FAFRM (respectively FCRM or FLB) affiliated with a $W^{*}$-probability space $(\mathcal{M}, \tau)$. Let further $A$ be a fixed set from $\sigma(\mathcal{E})$. Then the formulae

$$
M_{1}(E)=M(E \cap A), \quad \text { and } \quad M_{2}(E)=M(E \cap(X \backslash A)), \quad(E \in \mathcal{E}) \text {, }
$$

define new FAFRMs (respectively FCRMs or FLBs) on $(X, \mathcal{E})$, and $M_{1}$ and $M_{2}$ are freely independent in the sense that the $W^{*}$-algebras generated by the families $\left\{M_{1}(E) \mid E \in \mathcal{E}\right\}$ and $\left\{M_{2}(E) \mid E \in \mathcal{E}\right\}$ are freely independent.

Proof. Note first of all that (2.1) ensures that $M_{1}$ and $M_{2}$ are well-defined. Secondly, it is straightforward to check that $M_{1}$ and $M_{2}$ satisfy any of the conditions (a)-(d) in Definition 3.1, provided that $M$ satisfies that same condition. It remains therefore to argue that $M_{1}$ and $M_{2}$ are freely independent as stated, and for this it suffices to argue that the unital algebras $\mathcal{A}_{1}$ and $\mathcal{A}_{2}$ generated by $M_{1}$ and $M_{2}$, respectively, are freely independent (cf. [21, Proposition 2.5.7]). To validate the latter assertion it is sufficient to argue that any given finite subsets $\left\{a_{1}, \ldots, a_{n}\right\}$ of $\mathcal{A}_{1}$ and $\left\{b_{1}, \ldots, b_{m}\right\}$ of $\mathcal{A}_{2}$ generate freely independent unital subalgebras of $\mathcal{A}$. In this setup there exist finitely many sets $E_{1}, \ldots, E_{k}$ from $\mathcal{E}$ and functions $f_{1}, \ldots, f_{k}$ from $\mathcal{B F}_{b}(\mathbb{R})$, such that each $a_{j}$ is a (non-commutative) polynomial in (some of) the variables $f_{j}\left(M_{1}\left(E_{j}\right)\right), j=1, \ldots, k$. Since $\mathcal{E}$ is closed under intersections and set-differences, we can subsequently choose finitely many disjoint sets $F_{1}, \ldots, F_{l}$ from $\mathcal{E}$, such that each $E_{j}$ is a union of some of the $F_{i}$ 's. Let $\mathcal{B}_{1}$ denote the $W^{*}$-subalgebra of $\mathcal{A}$ generated by $\left\{M_{1}\left(F_{1}\right), \ldots, M_{1}\left(F_{l}\right)\right\}$. Then each $M_{1}\left(E_{j}\right)$ is affiliated with $\mathcal{B}_{1}$, being the sum of some of the $M_{1}\left(F_{i}\right)$ 's. In particular $f_{j}\left(M_{1}\left(E_{j}\right)\right) \in \mathcal{B}_{1}$ for all $j$, and hence also $a_{1}, \ldots, a_{n} \in \mathcal{B}_{1}$. Similarly there exists a finite family $G_{1}, \ldots, G_{r}$ of disjoint sets from $\mathcal{E}$, such that $b_{1}, \ldots, b_{m} \in \mathcal{B}_{2}$, with $\mathcal{B}_{2}$ being the $W^{*}$-subalgebra of $\mathcal{A}$ generated by $\left\{M_{2}\left(G_{1}\right), \ldots, M_{2}\left(G_{r}\right)\right\}$. Now, by Definition 3.1(a) and the definitions of $M_{1}$ and $M_{2}$ the operators

$$
M_{1}\left(F_{1}\right), \ldots, M_{1}\left(F_{l}\right), M_{2}\left(G_{1}\right), \ldots, M_{2}\left(G_{r}\right)
$$

are freely independent, and hence $\mathcal{B}_{1}$ and $\mathcal{B}_{2}$ are also freely independent (cf. [21, Proposition 2.5.5]). Obviously this further entails that the unital algebras generated by $\left\{a_{1}, \ldots, a_{n}\right\}$ and $\left\{b_{1}, \ldots, b_{r}\right\}$, respectively, are freely independent as well.

\subsection{Decomposition of a positive FCRM}

In this subsection we consider a non-empty set $X$ equipped with a $\delta$-ring $\mathcal{E}$ and a FCRM $M=\{M(E) \mid E \in \mathcal{E}\}$ affiliated with a $W^{*}$-probability space $(\mathcal{M}, \tau)$. We assume throughout that $M(E)$ is positive for all $E$ in $\mathcal{E}$ in the sense that $\operatorname{sp}(M(E)) \subseteq$ 
$[0, \infty)$, or, equivalently, that $\operatorname{supp}\left(L^{\mathrm{sp}}\{M(E)\}\right) \subseteq[0, \infty)$ for all $E \in \mathcal{E}$. We shall derive a decomposition of $M$ into the sum of an "atomic" FCRM and a FLB, a kin to the fundamental decomposition obtained by Kingman in [11] for classical completely random measures (CRM). The latter was obtained via the Laplace transforms of the considered CRM, which give rise to a positive measure on the underlying measurable space. This approach cannot directly be transferred to the non-commutative operator setting, since the formula $\exp (A+B)=\exp (A) \exp (B)$ does not generally hold for selfadjoint operators $A$ and $B$, unless they commute. Our construction given below therefore follows a different but related path, where the mentioned Laplace transforms are replaced by the set function

$$
\mu(E):=\int_{0}^{\infty} t L^{\mathrm{sp}}\{M(E)\}(\mathrm{d} t), \quad(E \in \mathcal{E}),
$$

which we shall argue is a measure on $\mathcal{E}$. In case $M(E)$ is bounded (and hence an element of $\mathcal{M})$ it holds automatically that $\mu(E)<\infty$, since the appearing integral equals $\tau(M(E)$ ). In general, when it is finite, $\mu(E)$ may also be identified with the first free cumulant of $L^{\mathrm{sp}}\{M(E)\}$ (cf. (2.8)), which we shall mostly denote simply by $\kappa_{1}(M(E))$ rather than $\kappa_{1}\left(L^{\mathrm{sp}}\{M(E)\}\right)$ to avoid too heavy notation.

Lemma 3.5. In the setting described above the formula

$$
\mu(E)=\int_{0}^{\infty} t L^{\mathrm{sp}}\{M(E)\}(\mathrm{d} t), \quad(E \in \mathcal{E})
$$

defines a (positive) measure on $(X, \mathcal{E})$.

Proof. Throughout this proof we employ for brevity the notation $\nu_{E}$ for the spectral distribution $L^{\operatorname{sp}}\{M(E)\}$ for any $E$ from E. From Definition 3.1(b) it follows that $M(\emptyset)=0$, so that $\nu_{\emptyset}=\delta_{0}$, and hence $\mu(\emptyset)=0$.

We show next that $\mu$ is finitely additive on $\mathcal{E}$. For this note first that if $A, B$ are sets from $\mathcal{E}$, such that $A \subseteq B$, then $M(A) \leq M(B)$, since $M(B)-M(A)=M(B \backslash A)$ is positive. According to Lemma 3.3 in [7] this means that the distribution functions $F_{\nu_{A}}$ and $F_{\nu_{B}}$ of $\nu_{A}$ and $\nu_{B}$ satisfy that $F_{\nu_{A}}(t) \geq F_{\nu_{B}}(t)$ for all $t$ in $\mathbb{R}$. For any $K$ in $[0, \infty)$ this further implies that

$$
\begin{aligned}
\int_{(K, \infty)} t \nu_{A}(\mathrm{~d} t) & =\int_{0}^{\infty} \nu_{A}\left(\left\{t \in \mathbb{R} \mid t 1_{(K, \infty)}(t)>s\right\}\right) \mathrm{d} s \\
& =\int_{0}^{\infty} \nu_{A}((K, \infty) \cap(s, \infty)) \mathrm{d} s=\int_{0}^{\infty}\left(1-F_{\nu_{A}}(K \vee s)\right) \mathrm{d} s \\
& \leq \int_{0}^{\infty}\left(1-F_{\nu_{B}}(K \vee s)\right) \mathrm{d} s=\int_{(K, \infty)} t \nu_{B}(\mathrm{~d} t)
\end{aligned}
$$

Consider now two disjoint sets $E_{1}, E_{2}$ from $\mathcal{E}$. If $\mu\left(E_{1}\right)=\infty$ or $\mu\left(E_{2}\right)=\infty$, then by (3.4) (in the case $K=0$ ) it follows that $\mu\left(E_{1} \cup E_{2}\right) \geq \mu\left(E_{1}\right) \vee \mu\left(E_{2}\right)=\infty$, and hence $\mu\left(E_{1} \cup E_{2}\right)=\mu\left(E_{1}\right)+\mu\left(E_{2}\right)$ in this case. If $\mu\left(E_{1}\right), \mu\left(E_{2}\right)<\infty$, then since $M\left(E_{1}\right)$ and $M\left(E_{2}\right)$ are freely independent, and since the free cumulants linearize free convolution (cf. (2.9)), it follows that

$$
\begin{aligned}
\mu\left(E_{1} \cup E_{2}\right) & =\kappa_{1}\left(M\left(E_{1} \cup E_{2}\right)\right)=\kappa_{1}\left(M\left(E_{1}\right)+M\left(E_{2}\right)\right)=\kappa_{1}\left(\nu_{E_{1}} \boxplus \nu_{E_{2}}\right) \\
& =\kappa_{1}\left(\nu_{E_{1}}\right)+\kappa_{1}\left(\nu_{E_{2}}\right)=\mu\left(E_{1}\right)+\mu\left(E_{2}\right) .
\end{aligned}
$$

Consider finally a sequence $\left(E_{n}\right)_{n \in \mathbb{N}}$ of sets from $\mathcal{E}$ such that $E:=\bigcup_{n \in \mathbb{N}} E_{n} \in \mathcal{E}$. We must show that $\mu(E)=\sum_{n=1}^{\infty} \mu\left(E_{n}\right)$. Recall first from Remark 3.2(2) that $M\left(\bigcup_{j=1}^{n} E_{j}\right) \rightarrow M(E)$ 
in the measure topology as $n \rightarrow \infty$. In particular $\nu_{\cup_{j=1}^{n} E_{j}} \rightarrow \nu_{E}$ weakly as $n \rightarrow \infty$ (cf. (2.5)), and this further entails that

$$
\begin{aligned}
\mu(E) & =\int_{0}^{\infty} t \nu_{E}(\mathrm{~d} t) \leq \liminf _{n \rightarrow \infty} \int_{0}^{\infty} t \nu_{\cup_{j=1}^{n} E_{j}}(\mathrm{~d} t)=\liminf _{n \rightarrow \infty} \mu\left(\bigcup_{j=1}^{n} E_{j}\right) \\
& =\liminf _{n \rightarrow \infty} \sum_{j=1}^{n} \mu\left(E_{j}\right)=\sum_{n=1}^{\infty} \mu\left(E_{n}\right),
\end{aligned}
$$

where we also invoked the finite additivity of $\mu$ established above. By (3.5) we may assume in the following that $\mu(E)<\infty$, and by (3.4) this further entails that $\mu\left(E_{n}\right) \leq$ $\mu\left(\bigcup_{j=1}^{n} E_{j}\right)<\infty$ for all $n$. From the finite additivity of $\mu$ we have that

$$
\mu(E)-\sum_{j=1}^{n} \mu\left(E_{j}\right)=\mu\left(E \backslash \bigcup_{j=1}^{n} E_{j}\right)
$$

for all $n$. Setting $G_{n}=E \backslash \bigcup_{j=1}^{n} E_{j}$ for all $n$, it suffices thus to show that

$$
\mu\left(G_{n}\right)=\int_{0}^{\infty} t \nu_{G_{n}}(\mathrm{~d} t) \longrightarrow 0 \text { as } n \rightarrow \infty .
$$

Since $G_{N} \downarrow \emptyset$ as $n \rightarrow \infty$, we know from condition (c) in Definition 3.1 that $\nu_{G_{n}} \stackrel{\text { w }}{\rightarrow} \delta_{0}$, and hence it is well-known that the convergence in (3.6) is equivalent to the condition that the family $\left\{\nu_{G_{n}} \mid n \in \mathbb{N}\right\}$ is uniformly integrable in the sense that

$$
\forall \epsilon \in(0, \infty) \exists K \in(0, \infty): \sup _{n \in \mathbb{N}} \int_{(K, \infty)} t \nu_{G_{n}}(\mathrm{~d} t) \leq \epsilon
$$

From (3.4) we have for any $n$ in $\mathbb{N}$ and $K$ in $[0, \infty)$ that

$$
\int_{(K, \infty)} t \nu_{G_{n}}(\mathrm{~d} t) \leq \int_{(K, \infty)} t \nu_{G_{1}}(\mathrm{~d} t)
$$

Since the right hand side does not depend on $n$ and converges to 0 as $K \rightarrow \infty$ (by dominated convergence) it follows readily that (3.7) is satisfied.

As described in Subsection 2.1 the measure $\mu$ introduced in Lemma 3.5 can be extended to a (positive) measure on $\sigma(\mathcal{E})$, which we also denote by $\mu$. We shall assume in the following that $\mu$ is $\sigma$-finite. This assumption may be seen as an analog of the (less restrictive) condition " $\mathcal{C}$ " presupposed in [11]. In the remainder of this section we shall assume further that the $\delta$-ring $\mathcal{E}$ satisfies condition (2.2).

Recall that an atom for $\mu$ is a set $A$ from $\sigma(\mathcal{E})$, such that $\mu(A)>0$ and $\mu(B \cap A) \in$ $\{0, \mu(A)\}$ for any set $B$ from $\sigma(\mathcal{E})$. It is well-known that any $\sigma$-finite measure may be decomposed into the sum of a purely atomic part and an atom-less part. More specifically there exists a subset $I$ of $\mathbb{N}$ and a corresponding family $\left(A_{n}\right)_{n \in I}$ of disjoint atoms for $\mu$, such that if we put $\mathfrak{A}=\bigcup_{n \in I} A_{n}$, and

$$
\mu_{c}(B):=\mu(B \cap(X \backslash \mathfrak{A})), \quad(B \in \sigma(\mathcal{E})),
$$

then the measure $\mu_{c}$ does not have any atoms. The "atomic part" of $\mu$ is then concentrated to the measure

$$
\mu_{a}(B):=\mu(B \cap \mathfrak{A})=\sum_{n \in I} \mu\left(B \cap A_{n}\right), \quad(B \in \sigma(\mathcal{E}))
$$


and the mentioned decomposition is

$$
\mu=\mu_{a}+\mu_{c}
$$

Note that the $\sigma$-finiteness of $\mu$ prevents any atom of $\mu$ from having infinite $\mu$-measure. In particular

$$
\mu\left(A_{n}\right) \in(0, \infty) \text { for all } n \text { in } \mathbb{N} .
$$

By a theorem of W. Sierpiński, the atom-free part $\mu_{c}$ has the following property: Any set $B$ from $\sigma(\mathcal{E})$, such that $0<\mu_{c}(B)<\infty$, admits for any $n$ in $\mathbb{N}$ a decomposition $B=\bigcup_{j=1}^{n} B_{j}$ into disjoint sets $B_{1}, \ldots, B_{n}$ from $\sigma(\mathcal{E})$, such that

$$
\mu_{c}\left(B_{j}\right)=\frac{\mu_{c}(B)}{n}, \quad j=1, \ldots, n .
$$

Corresponding to (3.8) we consider now the decomposition $M=M_{a}+M_{c}$, where

$$
M_{a}(E):=M(E \cap \mathfrak{A}), \quad \text { and } \quad M_{c}(E):=M(E \cap(X \backslash \mathfrak{A})) \text { for any } E \text { in } \mathcal{E} .
$$

We then have the following result.

Theorem 3.6. Let $X$ be a non-empty set, and let $\mathcal{E}$ be a $\delta$-ring in $X$ satisfying condition (2.2). Let further $M=\{M(E) \mid E \in \mathcal{E}\}$ be a positive FCRM on $(X, \mathcal{E})$, satisfying that the measure $\mu$ introduced in Lemma 3.5 is $\sigma$-finite, and consider the decomposition

$$
M(E)=M_{a}(E)+M_{c}(E), \quad(E \in \mathcal{E}),
$$

described above. Then $M_{c}$ and $M_{a}$ are freely independent, $M_{c}$ is a free Lévy basis, and there exists a countable family $\left(T_{n}\right)_{n \in I}$ of operators from $\left\{M_{a}(E) \mid E \in \mathcal{E}\right\}$, such that

$$
M_{a}(E)=\sum_{n \in I} \frac{\mu\left(A_{n} \cap E\right)}{\mu\left(A_{n}\right)} T_{n}, \quad(E \in \mathcal{E}),
$$

where $\left(A_{n}\right)_{n \in I}$ is the family of disjoint atoms for $\mu$ described above.

Concerning formula (3.12), note that $\frac{\mu\left(A_{n} \cap E\right)}{\mu\left(A_{n}\right)} \in\{0,1\}$ for any $E$ in $\sigma(\mathcal{E})$ and any $n$ in $I$, since $A_{n}$ is an atom for $\mu$. Note also that the sum converges in the measure topology in case $I$ is infinite (cf. Lemma 3.4 and Remark 3.2(2)).

Proof of Theorem 3.6. It follows directly from Lemma 3.4 that $M_{a}$ and $M_{c}$ defined by (3.11) are freely independent FCRM's on $(X, \mathcal{E})$. In order to prove that $M_{c}$ is a FLB, it remains then to verify that $L^{\mathrm{sp}}\left\{M_{c}(E)\right\} \in \mathcal{I D}(\boxplus)$ for any given $E$ from $\mathcal{E}$. We assume first that $\mu_{c}(E)<\infty$. As described above we may then, for any $n$ in $\mathbb{N}$, choose disjoint sets $E_{1}^{(n)}, \ldots, E_{n}^{(n)}$ from $\sigma(\mathcal{E})$, such that $E=\bigcup_{j=1}^{n} E_{j}^{(n)}$, and $\mu_{c}\left(E_{j}^{(n)}\right)=\frac{\mu_{c}(E)}{n}, j=1, \ldots, n$ (cf. (3.10)). From (2.1) it follows in particular that $E_{j}^{(n)} \in \mathcal{E}$ for all $j, n$, and therefore

$$
L^{\mathrm{sp}}\left\{M_{c}(E)\right\}=L^{\mathrm{sp}}\left\{M_{c}\left(E_{1}^{(n)}\right)+\cdots+M_{c}\left(E_{n}^{(n)}\right)\right\}=L^{\mathrm{sp}}\left\{M_{c}\left(E_{1}^{(n)}\right)\right\} \boxplus \cdots \boxplus L^{\mathrm{sp}}\left\{M_{c}\left(E_{n}^{(n)}\right)\right\} .
$$

Appealing now to [6, Theorem 1] it suffices to prove that the family $\left\{L^{\mathrm{sp}}\left\{M_{c}\left(E_{j}^{(n)}\right)\right\} \mid n \in\right.$ $\mathbb{N}, j=1, \ldots, n\}$ is a null-array in the sense that

$$
\forall \epsilon \in(0, \infty): \max _{1 \leq j \leq n} L^{\mathrm{sp}}\left\{M_{c}\left(E_{j}^{(n)}\right)\right\}\left([-\epsilon, \epsilon]^{c}\right) \underset{n \rightarrow \infty}{\longrightarrow} 0 .
$$

Given $\epsilon$ in $(0, \infty)$ it follows from Markov's Inequality that

$$
\begin{aligned}
L^{\mathrm{sp}}\left\{M_{c}\left(E_{j}^{(n)}\right)\right\}\left([-\epsilon, \epsilon]^{c}\right) & =L^{\mathrm{sp}}\left\{M_{c}\left(E_{j}^{(n)}\right)\right\}((\epsilon, \infty)) \leq \frac{1}{\epsilon} \int_{0}^{\infty} t L^{\mathrm{sp}}\left\{M\left(E_{j}^{(n)} \backslash \mathfrak{A}\right)\right\}(\mathrm{d} t) \\
& =\frac{1}{\epsilon} \mu\left(E_{j}^{(n)} \backslash \mathfrak{A}\right)=\frac{1}{\epsilon} \mu_{c}\left(E_{j}^{(n)}\right)=\frac{1}{n \epsilon} \mu_{c}(E)
\end{aligned}
$$


for any $j, n$. Since the resulting expression does not depend on $j$, this validates (3.13).

Assume next that $\mu_{c}(E)=\infty$. Since $\mu_{c}$ is $\sigma$-finite, we may choose a sequence $\left(E_{n}\right)_{n \in \mathbb{N}}$ of disjoint sets from $\sigma(\mathcal{E})$, such that $E=\bigcup_{n \in \mathbb{N}} E_{n}$ and $\mu_{c}\left(E_{n}\right)<\infty$ for all $n$. By (2.1) we have that $E_{n} \in \mathcal{E}$ for all $n$, and the argument above then ensures that $L^{\mathrm{sp}}\left\{M_{c}\left(E_{n}\right)\right\} \in \mathcal{J D}(\boxplus)$ for all $n$. Furthermore Remark 3.2(2) in conjunction with (2.5) yield that

$$
L^{\mathrm{sp}}\left\{M_{c}\left(E_{1}\right)\right\} \boxplus \cdots \boxplus L^{\mathrm{sp}}\left\{M_{c}\left(E_{n}\right)\right\}=L^{\mathrm{sp}}\left\{\sum_{j=1}^{n} M_{c}\left(E_{j}\right)\right\} \underset{n \rightarrow \infty}{\stackrel{\mathrm{w}}{\longrightarrow}} L^{\mathrm{sp}}\left\{M_{c}(E)\right\} .
$$

Since $\mathcal{I D}(\boxplus)$ is closed under free convolution and weak convergence, this yields that $L^{\mathrm{sp}}\left\{M_{c}(E)\right\} \in \mathcal{J D}(\boxplus)$ also in this case.

It remains to establish (3.12). By Remark 3.2(2) we note first for any $E$ in $\mathcal{E}$ that

$$
M_{a}(E)=M(E \cap \mathfrak{A})=\sum_{n \in I} M\left(E \cap A_{n}\right), \quad \text { where } \mu\left(E \cap A_{n}\right) \in\left\{0, \mu\left(A_{n}\right)\right\} \text { for all } n .
$$

In case $0=\mu\left(E \cap A_{n}\right)=\int_{0}^{\infty} t L^{\mathrm{sp}}\left\{M\left(E \cap A_{n}\right)\right\}(\mathrm{d} t)$, it follows that $L^{\mathrm{sp}}\left\{M\left(E \cap A_{n}\right)\right\}=\delta_{0}$, and hence $M\left(E \cap A_{n}\right)=0$, since $\tau$ is faithful. In order to establish (3.12) it suffices therefore to verify that

$$
M\left(E \cap A_{n}\right)=M\left(E^{\prime} \cap A_{n}\right)
$$

whenever $E, E^{\prime} \in \mathcal{E}$ such that $\mu\left(E \cap A_{n}\right)=\mu\left(A_{n}\right)=\mu\left(E^{\prime} \cap A_{n}\right)$. But given such $E, E^{\prime}$, note that (cf. (3.9))

$$
\mu\left(A_{n} \cap E \backslash E^{\prime}\right) \leq \mu\left(A_{n} \backslash E^{\prime}\right)=\mu\left(A_{n}\right)-\mu\left(A_{n} \cap E^{\prime}\right)=0,
$$

and hence it follows as above by faithfulness of $\tau$ that $M\left(A_{n} \cap E \backslash E^{\prime}\right)=0$, and similarly that $M\left(A_{n} \cap E^{\prime} \backslash E\right)=0$. Therefore

$$
M\left(A_{n} \cap E\right)=M\left(A_{n} \cap E \cap E^{\prime}\right)+M\left(A_{n} \cap E \backslash E^{\prime}\right)=M\left(A_{n} \cap E \cap E^{\prime}\right)=M\left(A_{n} \cap E^{\prime}\right),
$$

as desired. This completes the proof.

\subsection{Decomposition of a signed FCRM}

Let $\mathcal{E}$ be a $\delta$-ring on a non-empty set $X$, and let $M=\{M(E) \mid E \in \mathcal{E}\}$ be a FCRM. In this subsection we establish a decomposition similar to that obtained in the previous subsection in the more general situation, where we drop the assumption of positivity. The corresponding problem for "signed" CRMs was not considered by Kingman; presumably because the approach using Laplace transforms is not directly applicable. Our approach to the case of "signed" FCRMs requires stronger moment conditions than those considered in the positive case, where $\sigma$-finiteness of the measure introduced in Lemma 3.5 was presupposed. Specifically we require in the following existence of second moments, i.e.

$$
\int_{\mathbb{R}} t^{2} L^{\mathrm{sp}}\{M(E)\}(\mathrm{d} t)<\infty \quad \text { for any } E \text { in } \mathcal{E},
$$

but we shall actually need slightly more than that (see Lemma 3.7 and Remark 3.8 below). The existence of second moments allows us to consider the second free cumulant (cf. (2.8))

$$
\kappa_{2}\left(L^{\mathrm{sp}}\{M(E)\}\right)=\int_{\mathbb{R}} t^{2} L^{\mathrm{sp}}\{M(E)\}(\mathrm{d} t)-\left(\int_{\mathbb{R}} t L^{\mathrm{sp}}\{M(E)\}(\mathrm{d} t)\right)^{2} \geq 0,
$$

which we denote for brevity by $\kappa_{2}(M(E))$. 
Lemma 3.7. Let $M=\{M(E) \mid E \in \mathcal{E}\}$ be a FCRM satisfying condition (3.14). Assume additionally that

$$
\lim _{n \rightarrow \infty} \kappa_{2}\left(M\left(E_{n}\right)\right)=0 \quad \text { for any sequence }\left(E_{n}\right)_{n \in \mathbb{N}} \text { from } \mathcal{E} \text {, such that } E_{n} \downarrow \emptyset .
$$

Then the formulae

$$
\begin{aligned}
& \mu_{1}(E)=\kappa_{1}(M(E))=\int_{\mathbb{R}} t L^{\mathrm{sp}}\{M(E)\}(\mathrm{d} t), \\
& \mu_{2}(E)=\kappa_{2}(M(E)),
\end{aligned}
$$

define, respectively, a signed measure $\mu_{1}$ and a positive measure $\mu_{2}$ on $(X, \mathcal{E})$.

Proof. As in the proof Lemma 3.5 it follows that $\mu_{1}(\emptyset)=\mu_{2}(\emptyset)=0$, and that $\mu_{1}$ and $\mu_{2}$ are finitely additive on $\mathcal{E}$, since $\kappa_{1}$ and $\kappa_{2}$ both linearize $\boxplus$. For a sequence $\left(D_{n}\right)_{n \in \mathbb{N}}$ of disjoint sets from $\mathcal{E}$, such that $D:=\bigcup_{n \in \mathbb{N}} D_{n} \in \mathcal{E}$, the finite additivity and condition (3.14) further ensure the validity of the calculation:

$$
\mu_{2}(D)-\sum_{j=1}^{n} \mu_{2}\left(D_{j}\right)=\mu_{2}\left(D \backslash \bigcup_{j=1}^{n} D_{j}\right)=\kappa_{2}\left(M\left(D \backslash \bigcup_{j=1}^{n} D_{j}\right)\right),
$$

and hence (3.16) supplies the remaining condition for $\mu_{2}$ to be a measure on $(X, \mathcal{E})$. In order to complete the proof it remains therefore only to verify that also

$$
\lim _{n \rightarrow \infty} \kappa_{1}\left(M\left(E_{n}\right)\right)=0 \quad \text { for any sequence }\left(E_{n}\right)_{n \in \mathbb{N}} \text { from } \mathcal{E} \text {, such that } E_{n} \downarrow \emptyset .
$$

Consider thus such a sequence $\left(E_{n}\right)_{n \in \mathbb{N}}$, and for brevity put $\nu_{n}=L^{\mathrm{sp}}\left\{M\left(E_{n}\right)\right\}$ for each $n$. Then Definition 3.1(c) entails that $\nu_{n} \stackrel{\mathrm{w}}{\rightarrow} \delta_{0}$ as $n \rightarrow \infty$. For any positive $\epsilon$ this, in conjunction with (3.16), leads to

$$
\begin{aligned}
\nu_{n}\left(\left\{t \in \mathbb{R}|| t-\kappa_{1}\left(\nu_{n}\right)|+| t \mid>2 \epsilon\right\}\right) & \leq \nu_{n}\left(\left\{t \in \mathbb{R}|| t-\kappa_{1}\left(\nu_{n}\right) \mid>\epsilon\right\}\right)+\nu_{n}\left([-\epsilon, \epsilon]^{c}\right) \\
& \leq \epsilon^{-2} \int_{\mathbb{R}}\left(t-\kappa_{1}\left(\nu_{n}\right)\right)^{2} \nu_{n}(\mathrm{~d} t)+\nu_{n}\left([-\epsilon, \epsilon]^{c}\right) \\
& =\epsilon^{-2} \kappa_{2}\left(\nu_{n}\right)+\nu_{n}\left([-\epsilon, \epsilon]^{c}\right) \underset{n \rightarrow \infty}{\longrightarrow} 0 .
\end{aligned}
$$

In particular $\left\{t \in \mathbb{R}|| t-\kappa_{1}\left(\nu_{n}\right)|+| t \mid \leq 2 \epsilon\right\} \neq \emptyset$ for all sufficiently large $n$, and for such $n$ we can choose $t_{n}$ in $\mathbb{R}$ such that $\left|t_{n}-\kappa_{1}\left(\nu_{n}\right)\right|+\left|t_{n}\right| \leq 2 \epsilon$. But then also $\left|\kappa_{1}\left(\nu_{n}\right)\right| \leq\left|\kappa_{1}\left(\nu_{n}\right)-t_{n}\right|+\left|t_{n}\right| \leq 2 \epsilon$, and this verifies (3.17).

Remark 3.8. The assumption (3.16) in Lemma 3.7 may appear rather "artificial", as it is essentially equivalent to the statement that $\mu_{2}$ is a measure. The proof of Lemma 3.7 shows that (3.16) implies that $\kappa_{1}\left(M\left(E_{n}\right)\right) \rightarrow 0$ as $n \rightarrow \infty$, and hence also that

$$
\int_{\mathbb{R}} t^{2} L^{\mathrm{sp}}\left\{M\left(E_{n}\right)\right\}(\mathrm{d} t)=\kappa_{2}\left(M\left(E_{n}\right)\right)+\kappa_{1}\left(M\left(E_{n}\right)\right)^{2} \underset{n \rightarrow \infty}{\longrightarrow} 0
$$

for any sequence $\left(E_{n}\right)_{n \in \mathbb{N}}$ from $\mathcal{E}$, such that $E_{n} \downarrow \emptyset$. Thus (3.16) is in fact - in the considered setup - equivalent to convergence to 0 in the square mean, which by standard results is equivalent to uniform integrability of the sequence

$$
\left\{L^{\mathrm{sp}}\left\{M\left(E_{n}\right)\right\} \circ \mathrm{sq}^{-1} \mid n \in \mathbb{N}\right\}
$$

of transformations of $L^{\mathrm{sp}}\left\{M\left(E_{n}\right)\right\}$ by the mapping sq: $x \mapsto x^{2}: \mathbb{R} \rightarrow \mathbb{R}$. Consequently a more elaborate condition on $M$, ensuring the validity of (3.16), is that the family 
$\left\{L^{\mathrm{sp}}\left\{M\left(E^{\prime}\right)\right\} \circ \mathrm{sq}^{-1} \mid E^{\prime} \in \mathcal{E}, E^{\prime} \subseteq E\right\}$ be uniformly integrable for any $E$ in $\mathcal{E}$. This latter condition is satisfied, in particular, if there exists a positive number $\epsilon$, such that

$$
\sup _{\substack{E^{\prime} \in \mathcal{E} \\ E^{\prime} \subseteq E}} \int_{\mathbb{R}}|t|^{2+\epsilon} L^{\mathrm{sp}}\left\{M\left(E^{\prime}\right)\right\}(\mathrm{d} t)<\infty \text { for any } E \text { in } \mathcal{E},
$$

and one could even allow for $\epsilon$ to depend on $E$.

In the setting of Lemma 3.7 we consider next the positive measure

$$
\mu=\left|\mu_{1}\right|+\mu_{2}
$$

where $\left|\mu_{1}\right|$ denotes the total variation measure of the signed measure $\mu_{1}$ (cf. Subsection 2.1). We extend $\mu$ to a measure on $\sigma(\mathcal{E})$ (also denoted $\mu$ ) and assume again that $(X, \mathcal{E})$ satisfies condition (2.2). In combination with (3.14) this entails that $\mu$ is $\sigma$-finite and hence it admits an atomic decomposition:

$$
\mu=\mu_{a}+\mu_{c}
$$

as described in Subsection 3.1. Specifically we introduce a countable family $\left(A_{n}\right)_{n \in I} \subseteq$ $\sigma(\mathcal{E})$ of disjoint atoms for $\mu$, such that

$$
\mu_{a}(B)=\mu(B \cap \mathfrak{A}) \quad \text { and } \quad \mu_{c}(B)=\mu(B \backslash \mathfrak{A}) \quad \text { for any } B \text { in } \sigma(\mathcal{E}),
$$

where $\mathfrak{A}=\bigcup_{n \in I} A_{n}$. We consider then the corresponding decomposition $M=M_{a}+M_{c}$ of $M$, where

$$
M_{a}(E)=M(E \cap \mathfrak{A}) \quad \text { and } \quad M_{c}(E)=M(E \backslash \mathfrak{A}) \quad \text { for any } E \text { in } \mathcal{E} .
$$

Theorem 3.9. Let $X$ be a non-empty set, and let $\mathcal{E}$ be a $\delta$-ring in $X$ satisfying condition (2.2). Let further $M=\{M(E) \mid E \in \mathcal{E}\}$ be a FCRM on $(X, \mathcal{E})$ affilliated with a $W^{*}$-probability space $(\mathcal{M}, \tau)$ and satisfying (3.14) and (3.16). Consider also the decomposition

$$
M(E)=M_{a}(E)+M_{c}(E), \quad(E \in \mathcal{E}),
$$

described above. Then $M_{c}$ and $M_{a}$ are freely independent, $M_{c}$ is a free Lévy basis, and there exists a countable family $\left(T_{n}\right)_{n \in I}$ of operators from $\left\{M_{a}(E) \mid E \in \mathcal{E}\right\}$, such that

$$
M_{a}(E)=\sum_{n \in I} \frac{\mu\left(A_{n} \cap E\right)}{\mu\left(A_{n}\right)} T_{n}, \quad(E \in \mathcal{E}) .
$$

Here $\mu$ is given by (3.18) and $\left(A_{n}\right)_{n \in I}$ is the family of disjoint atoms for $\mu$ described above.

Proof. The proof is similar to that of Theorem 3.6, and we shall not repeat all details. It follows directly from Lemma 3.4 that $M_{a}$ and $M_{c}$ are freely independent FCRM's. To show that $L^{\mathrm{sp}}\left\{M_{c}(E)\right\} \in \mathcal{J D}(\boxplus)$ for any $E$ in $\mathcal{E}$, we use the facts that $\mu_{c}$ is atom-less and that $\mu_{c}(E)<\infty$ to choose, for any $n$ in $\mathbb{N}$, disjoint sets $E_{1}^{(n)}, \ldots, E_{n}^{(n)}$ from $\mathcal{E}$, such that $E=\bigcup_{j=1}^{n} E_{j}^{(n)}$, and such that $\mu_{c}\left(E_{j}^{(n)}\right)=\frac{\mu_{c}(E)}{n}, j=1, \ldots, n$. Note then for any $n$ in $\mathbb{N}$ that

$$
L^{\mathrm{sp}}\left\{M_{c}(E)\right\}=\delta_{\mu_{1}(E \backslash \mathfrak{A})} \boxplus\left(\underset{j=1}{\boxplus^{\boxplus}} L^{\mathrm{sp}}\left\{M_{c}\left(E_{j}^{(n)}\right)-\mu_{1}\left(E_{j}^{(n)} \backslash \mathfrak{A}\right) \mathbf{1}_{\mathcal{M}}\right\}\right),
$$

where $\mathbf{1}_{\mathcal{M}}$ denotes the multiplicative unit of $\mathcal{M}$. By [6, Theorem 1] it suffices thus to show that

$$
\forall \epsilon \in(0, \infty): \max _{1 \leq j \leq n} L^{\mathrm{sp}}\left\{M_{c}\left(E_{j}^{(n)}\right)-\mu_{1}\left(E_{j}^{(n)} \backslash \mathfrak{A}\right) \mathbf{1}_{\mathcal{M}}\right\}\left([-\epsilon, \epsilon]^{c}\right) \underset{n \rightarrow \infty}{\longrightarrow} 0 .
$$


Given $\epsilon$ in $(0, \infty)$, we find for any $j, n$ by Chebyshev's Inequality that

$$
\begin{aligned}
L^{\mathrm{sp}}\left\{M_{c}\left(E_{j}^{(n)}\right)-\right. & \left.\mu_{1}\left(E_{j}^{(n)} \backslash \mathfrak{A}\right) \mathbf{1}_{\mathcal{M}}\right\}\left([-\epsilon, \epsilon]^{c}\right) \\
= & L^{\mathrm{sp}}\left\{M_{c}\left(E_{j}^{(n)}\right)\right\}\left(\left\{t \in \mathbb{R}|| t-\kappa_{1}\left(M_{c}\left(E_{j}^{(n)}\right)\right) \mid>\epsilon\right\}\right) \\
\leq & \epsilon^{-2} \kappa_{2}\left(M_{c}\left(E_{j}^{(n)}\right)\right)=\epsilon^{-2} \kappa_{2}\left(M\left(E_{j}^{(n)} \backslash \mathfrak{A}\right)\right) \\
\leq & \epsilon^{-2} \mu\left(E_{j}^{(n)} \backslash \mathfrak{A}\right)=\epsilon^{-2} \mu_{c}\left(E_{j}^{(n)}\right)=\frac{\mu_{c}(E)}{n \epsilon^{2}}
\end{aligned}
$$

from which (3.20) follows readily. It remains to verify (3.19). Note initially that

$$
M_{a}(E)=M(E \cap \mathfrak{A})=\sum_{n \in I} M\left(E \cap A_{n}\right)
$$

for any $E$ in $\mathcal{E}$ by (2.1) and Remark 3.2(2). If $\mu\left(E \cap A_{n}\right)=0$, then in particular the variance $\kappa_{2}\left(M\left(E \cap A_{n}\right)\right)=0$, and hence $L^{\mathrm{sp}}\left\{M_{a}\left(E \cap A_{n}\right)\right\}=\delta_{c}$ for some $c \in \mathbb{R}$. Since also $\left|\mu_{1}\right|\left(E \cap A_{n}\right)=0$, and therefore $\kappa_{1}\left(M_{a}\left(E \cap A_{n}\right)\right)=\mu_{1}\left(E \cap A_{n}\right)=0$, we must then have that $c=0$. By faithfulness of $\tau$ this implies that $M\left(E \cap A_{n}\right)=0$. To verify (3.19) it suffices therefore to argue for any $n$ in $\mathbb{N}$ and any $E, E^{\prime}$ from $\mathcal{E}$ that

$$
\mu\left(E \cap A_{n}\right)=\mu\left(A_{n}\right)=\mu\left(E^{\prime} \cap A_{n}\right) \Longrightarrow M\left(E \cap A_{n}\right)=M\left(E^{\prime} \cap A_{n}\right)
$$

Assuming the left hand side of (3.21) it suffices as in the proof of Theorem 3.6 to show that $M\left(A_{n} \cap E \backslash E^{\prime}\right)=M\left(A_{n} \cap E^{\prime} \backslash E\right)=0$, and as argued above this follows by faithfulness of $\tau$, if we validate that $\mu\left(A_{n} \cap E \backslash E^{\prime}\right)=\mu\left(A_{n} \cap E^{\prime} \backslash E\right)=0$. But this follows exactly as in the proof of Theorem 3.6.

\section{Free Lévy bases}

For a Free Lévy basis the $\boxplus$-infinite divisibility of the marginals makes it possible to transfer major elements of the theory of classical Lévy bases, as developed in [16], to the free setting via the Bercovici-Pata bijection. Following that strategy we record in this section some basic results on free Lévy bases. The starting point is the following:

Theorem 4.1. Let $\mathcal{E}$ be a $\delta$-ring of subsets of a non-empty set $X$.

(i) For any free Lévy basis $M=\{M(E) \mid E \in \mathcal{E}\}$ (affiliated with some $W^{*}$-probability space) there exists a classical Lévy basis $N=\{N(E) \mid E \in \mathcal{E}\}$ defined on some probability space $(\Omega, \mathcal{F}, P)$, such that

$$
\Lambda(L\{N(E)\})=L^{\mathrm{sp}}\{M(E)\} \text { for all } E \text { in } \mathcal{E} .
$$

(ii) For any classical Lévy basis $N=\{N(E) \mid E \in \mathcal{E}\}$ (defined on some classical probability space) there exists a free Lévy basis $M=\{M(E) \mid E \in \mathcal{E}\}$ affiliated with some $W^{*}$-probability space $(\mathcal{M}, \tau)$, such that the relation (4.1) holds.

Proof. (i) Consider a free Lévy basis $M=\{M(E) \mid E \in \mathcal{E}\}$ affiliated with some $W^{*}$ probability space $(\mathcal{M}, \tau)$, and for each $E$ in $\mathcal{E}$ put

$$
\mu(E, \cdot)=\Lambda^{-1}\left(L^{\mathrm{sp}}\{M(E)\}\right) .
$$

If $E_{1}, \ldots, E_{n}$ are disjoint sets from $\mathcal{E}$ we have then that

$$
\begin{aligned}
\mu\left(\bigcup_{j=1}^{n} E_{j}, \cdot\right) & =\Lambda^{-1}\left(L^{\mathrm{sp}}\left\{M\left(E_{1}\right)+\cdots+M\left(E_{n}\right)\right\}\right) \\
& =\Lambda^{-1}\left(L^{\mathrm{sp}}\left\{M\left(E_{1}\right)\right\} \boxplus \cdots \boxplus L^{\mathrm{sp}}\left\{N\left(E_{n}\right)\right\}\right) \\
& =\Lambda^{-1}\left(L^{\mathrm{sp}}\left\{M\left(E_{1}\right)\right\}\right) * \cdots * \Lambda^{-1}\left(L^{\mathrm{sp}}\left\{M\left(E_{n}\right)\right\}\right) \\
& =\mu\left(E_{1}, \cdot\right) * \cdots * \mu\left(E_{n}, \cdot\right) .
\end{aligned}
$$


In addition, for any decreasing sequence $\left(F_{n}\right)_{n \in \mathbb{N}}$ from $\mathcal{E}$, such that $\bigcap_{n \in \mathbb{N}} F_{n}=\emptyset$, we have that $L^{\mathrm{sp}}\left\{M\left(F_{n}\right)\right\} \stackrel{\mathrm{w}}{\rightarrow} \delta_{0}$ as $n \rightarrow \infty$, and hence by continuity of $\Lambda^{-1}$,

$$
\mu\left(F_{n}, \cdot\right) \underset{n \rightarrow \infty}{\stackrel{\mathrm{w}}{\longrightarrow}} \delta_{0}
$$

It follows from (4.2) and the Kolmogorov Extension Theorem that there exists a finitely additive, infinitely divisible random measure $N=\{N(E) \mid E \in \mathcal{E}\}$, defined on some probability space $(\Omega, \mathcal{F}, P)$, such that $L\{N(E)\}=\mu(E, \cdot)$ for all $E$ in $\mathcal{E}$. If $\left(E_{n}\right)_{n \in \mathbb{N}}$ is a sequence of disjoint sets from $\mathcal{E}$, such that $E:=\bigcup_{n \in \mathbb{N}} E_{n} \in \mathcal{E}$, then (4.3) implies that $\sum_{j=1}^{n} N\left(E_{j}\right) \rightarrow N(E)$ in probability as $n \rightarrow \infty$. Since the terms $N\left(E_{1}\right), N\left(E_{2}\right), N\left(E_{3}\right), \ldots$ are independent, the convergence also holds almost surely. Hence $N$ is a classical Lévy basis.

(ii) Let $N=\{N(E) \mid E \in \mathcal{E}\}$ be a classical Lévy basis defined on some probability space $(\Omega, \mathcal{F}, P)$, and for any $E$ in $\mathcal{E}$ put

$$
\nu(E, \cdot)=\Lambda(L\{N(E)\}) \in \mathcal{J D}(\boxplus) .
$$

Argumentation similar to that of the proof of (i) verifies that the family $\{\nu(E, \cdot) \mid E \in \mathcal{E}\}$ satisfies (3.2) and the conditions in (ii) and (iii) of Theorem 3.3. Hence that same theorem provides the existence of a FLB with the described properties.

Next we transfer some fundamental results from [16] on classical Lévy bases to corresponding results for free Lévy bases. In the remaining part of this section we consider thus, as in [16], a $\delta$-ring $\mathcal{E}$ in $X$ satisfying condition (2.2). Note that without loss of generality we may assume that the $U_{n}$ 's from (2.2) are disjoint or increasing in $n$.

Proposition 4.2. (i) Let $M=\{M(E) \mid E \in \mathcal{E}\}$ be a free Lévy basis affiliated with some $W^{*}$-probability space $(\mathcal{M}, \tau)$. Then there exist a signed measure $\Theta: \mathcal{E} \rightarrow \mathbb{R}$, a finite (positive) measure $\Sigma: \mathcal{E} \rightarrow[0, \infty)$ and a $\sigma$-finite (positive) measure $F: \sigma(\mathcal{E}) \otimes$ $\mathcal{B}(\mathbb{R}) \rightarrow[0, \infty]$ such that the free Lévy-Khintchine representation of $M(E)$ is given by

$$
\mathcal{C}_{M(E)}(z)=z \Theta(E)+z^{2} \Sigma(E)+\int_{\mathbb{R}}\left(\frac{1}{1-t z}-1-z \varsigma(t)\right) F_{E}(\mathrm{~d} t), \quad\left(z \in \mathbb{C}^{-}\right),
$$

for any $E$ in $\mathcal{E}$. Here $F_{E}$ is the measure on $\mathcal{B}(\mathbb{R})$ given by: $F_{E}(B)=F(E \times B)$ for any $B$ in $\mathcal{B}(\mathbb{R})$, and $F_{E}$ is a Lévy measure on $\mathbb{R}$ for all $E$ in $\mathcal{E}$.

(ii) For any triplet $(\Theta, \Sigma, F)$ of measures as described in (i), there exists a free Lévy basis $M=\{M(E) \mid E \in \mathcal{E}\}$ (affiliated with some $W^{*}$-probability space), such that (4.4) holds.

(iii) Let $M$ and $(\Theta, \Sigma, F)$ be as stated in (i). Then there exists a unique, $\sigma$-finite and positive measure $\kappa$ on $\sigma(\mathcal{E})$ with the following properties:

(a) $\kappa(E)=|\Theta|(E)+\Sigma(E)+\int_{\mathbb{R}} \min \left\{1, x^{2}\right\} F_{E}(\mathrm{~d} x)$ for all $E$ in $\mathcal{E}$.

(b) If $\left(E_{n}\right)_{n \in \mathbb{N}}$ is a sequence of sets from $\mathcal{E}$, such that $\kappa\left(E_{n}\right) \rightarrow 0$ as $n \rightarrow \infty$, then $M\left(E_{n}\right) \rightarrow 0$ in the measure topology.

(c) Suppose $\left(E_{n}\right)_{n \in \mathbb{N}}$ is a sequence of sets from $\mathcal{E}$, such that $M\left(E_{n}^{\prime}\right) \rightarrow 0$ in the measure topology for any sequence $\left(E_{n}^{\prime}\right)_{n \in \mathbb{N}}$ from $\mathcal{E}$, such that $E_{n}^{\prime} \subseteq E_{n}$ for all $n$. Then $\kappa\left(E_{n}\right) \rightarrow 0$ as $n \rightarrow \infty$.

The triplet $(\Theta, \Sigma, F)$ of measures introduced in Proposition 4.2(i) is referred to as the free characteristic triplet of the free Lévy basis $M$. The measure $\kappa$ is referred to as the control measure of $M$. 
For the measure $F_{E}$ in 4.2(i) it follows e.g. by a standard extension argument that a Borel function $f: \mathbb{R} \rightarrow \mathbb{C}$ is in $\mathcal{L}^{1}\left(F_{E}\right)$, if and only if $1_{E} \otimes f \in \mathcal{L}^{1}(F)$, in which case

$$
\int_{\mathbb{R}} f(t) F_{E}(\mathrm{~d} t)=\int_{X \times \mathbb{R}} 1_{E}(x) f(t) F(\mathrm{~d} x, \mathrm{~d} t) .
$$

We note also that the measure $F$ is uniquely determined on the $\sigma$-algebra $\sigma(\mathcal{E}) \otimes \mathcal{B}(\mathbb{R})$ by the condition: $F(E \times B)=F_{E}(B)$ for all $E$ in $\mathcal{E}$ and $B$ in $\mathcal{B}(\mathbb{R})$, since this also implies that $F\left(U_{n} \times\left(\mathbb{R} \backslash\left[-\frac{1}{n}, \frac{1}{n}\right]\right)\right)<\infty$ for all $n$, because $F_{U_{n}}$ is a Lévy measure. Here $\left(U_{n}\right)_{n \in \mathbb{N}}$ is the sequence from (2.2), chosen to be increasing.

Proof of Proposition 4.2. (i) Let $N=\{N(E) \mid E \in \mathcal{E}\}$ be a classical Lévy basis corresponding to $M$ as described in Theorem 4.1. Then by Proposition 2.1 and Lemma 2.3 in [16] there exist a signed measure $\Theta: \mathcal{E} \rightarrow \mathbb{R}$, a finite measure $\Sigma: \mathcal{E} \rightarrow[0, \infty)$ and a $\sigma$-finite measure $F: \sigma(\mathcal{E}) \otimes \mathcal{B}(\mathbb{R}) \rightarrow[0, \infty]$, such that

$$
C_{N(E)}(y)=\mathrm{i} y \Theta(E)-\frac{1}{2} y^{2} \Sigma(E)+\int_{\mathbb{R}}\left(\mathrm{e}^{\mathrm{i} t y}-1-\mathrm{i} y \varsigma(t)\right) F_{E}(\mathrm{~d} t), \quad(y \in \mathbb{R}),
$$

for all $E$ in $\mathcal{E}$. Since $L^{\operatorname{sp}}\{M(E)\}=\Lambda(L\{N(E)\})$ for all $E$ in $\mathcal{E}$, it follows immediately from the definition of $\Lambda$ that $(\Theta, \Sigma, F)$ satisfies (4.4) as well.

(ii) If $(\Theta, \Sigma, F)$ is a triplet as described in (i), then Proposition 2.1 in [16] ensures the existence of a classical Lévy basis $N=\{N(E) \mid E \in \mathcal{E}\}$ such that (4.6) holds. Subsequently Theorem 4.1 provides a free Lévy basis $M=\{M(E) \mid E \in \mathcal{E}\}$ such that (4.4) holds.

(iii) Let $N$ be as in the proof of (i). It follows then from Proposition 2.1 in [16] that there exists a $\sigma$-finite measure $\kappa$ on $\sigma(\varepsilon)$, such that (a) is satisfied, and such that (b) and (c) hold with $M$ replaced by $N$ and convergence in the measure topology replaced by convergence in probability. Since e.g. in (b) $M\left(E_{n}\right) \rightarrow 0$ in the measure topology, if and only if $N\left(E_{n}\right) \rightarrow 0$ in probability, it follows readily that $\kappa$ satisfies (b) and (c) as they stand.

Since the triplet $(\Theta, \Sigma, F)$ appearing in Proposition 4.2(i) is obtained by application of Proposition 2.1 in [16] to a classical Lévy basis, it follows immediately from Lemma 2.3 of that same paper, that there exists a mapping $\rho: X \times \mathcal{B}(\mathbb{R}) \rightarrow[0, \infty]$ with the following properties:

(i) $\rho(x, \cdot)$ is a Lévy measure on $\mathbb{R}$ for any fixed $x$ in $X$.

(ii) $\rho(\cdot, B)$ is a $\sigma(\mathcal{E})$-measurable function for any fixed Borel subset $B$ of $\mathbb{R}$.

(iii) For any function $h: X \times \mathbb{R} \rightarrow \mathbb{C}$ which is positive and $\sigma(\mathcal{E}) \otimes \mathcal{B}(\mathbb{R})$-measurable or in $\mathcal{L}^{1}(F)$ it holds that

$$
\int_{X \times \mathbb{R}} h(x, t) F(\mathrm{~d} x, \mathrm{~d} t)=\int_{X}\left(\int_{\mathbb{R}} h(x, t) \rho(x, \mathrm{~d} t)\right) \kappa(\mathrm{d} x),
$$

where $\kappa$ is the control measure introduced in Proposition 4.2(iii), and the integral $\int_{\mathbb{R}} h(x, t) \rho(x, \mathrm{~d} t)$ is well-defined for $\kappa$-almost all $x$ in $X$.

We note also that $\rho$ is unique up to $\kappa$-null-sets: If $\rho^{\prime}: X \times \mathbb{R} \rightarrow[0, \infty]$ is another mapping satisfying conditions (i)-(iii), then $\rho(x, \cdot)=\rho^{\prime}(x, \cdot)$ for $\kappa$-almost all $x$, since $\mathcal{B}(\mathbb{R})$ is countably generated.

For the mapping $\rho$ we now have the following analog of Proposition 2.4 in [16]. 
Proposition 4.3. Consider a free Lévy basis $M=\{M(E) \mid E \in \mathcal{E}\}$, and let $\Theta, \Sigma, F, \kappa$ be the associated measures described in Proposition 4.2. Furthermore let $\rho$ be the corresponding mapping introduced above, and let $\theta$ and $\sigma^{2}$ denote, respectively, the Radon-Nikodym derivatives of $\Theta$ and $\Sigma$ with respect to $\kappa$. Then for any set $E$ from $\mathcal{E}$ we have the formula:

$$
\mathcal{C}_{M(E)}(z)=\int_{E} R(x, z) \kappa(\mathrm{d} x), \quad\left(z \in \mathbb{C}^{-}\right)
$$

where the kernel $R(\cdot, \cdot)$ is given by

$$
R(x, z)=z \theta(x)+z^{2} \sigma^{2}(x)+\int_{\mathbb{R}}\left(\frac{1}{1-t z}-1-z \varsigma(t)\right) \rho(x, \mathrm{~d} t),
$$

for all $z$ in $\mathbb{C}^{-}$and $x$ in $X$.

Proof. By the definition of $\kappa$ in 4.2(iii) it is clear that $|\Theta|, \Sigma \leq \kappa$, so that $\theta$ and $\sigma^{2}$ are well-defined (see Subsection 2.1). Let $E$ be a given set from $\mathcal{E}$. For any fixed $z$ in $\mathbb{C}^{-}$ the function $t \mapsto \frac{1}{1-t z}-1-z \varsigma(t)$ belongs to $\mathcal{L}^{1}\left(F_{E}\right)$ and to $\mathcal{L}^{1}(\rho(x, \cdot))$ for all $x$ in $X$, since the considered measures are all Lévy measures. Combining formulae (4.5) and (4.7) it follows further that

$$
\begin{aligned}
\int_{E}\left(\int_{\mathbb{R}}\left(\frac{1}{1-t z}-1-z \varsigma(t)\right) \rho(x, \mathrm{~d} t)\right) \kappa(\mathrm{d} x) & =\int_{X \times \mathbb{R}} 1_{E}(x)\left(\frac{1}{1-t z}-1-z \varsigma(t)\right) F(\mathrm{~d} x, \mathrm{~d} t) \\
& =\int_{\mathbb{R}}\left(\frac{1}{1-t z}-1-z \varsigma(t)\right) F_{E}(\mathrm{~d} t) .
\end{aligned}
$$

Therefore, by the definitions of $R, \theta$ and $\sigma^{2}$,

$$
\int_{E} R(x, z) \kappa(\mathrm{d} x)=z \Theta(E)+z^{2} \Sigma(E)+\int_{\mathbb{R}}\left(\frac{1}{1-t z}-1-z \varsigma(t)\right) F_{E}(\mathrm{~d} t)=\mathcal{C}_{M(E)}(z),
$$

where the last equality is (4.4).

Remark 4.4. For fixed $x$ in $X$ the "slice-function" $R(x, \cdot)$ of the kernel $R$ in Proposition 4.3 is the free cumulant transform of a freely infinitely divisible probability measure $\nu_{x}$ with free characteristic triplet $\left(\theta(x), \sigma^{2}(x), \rho(x, \cdot)\right)$. In the literature on classical Lévy bases the measure $\mu_{x}$ in $\mathcal{J D}(*)$ with classical characteristic triplet $\left(\theta(x), \sigma^{2}(x), \rho(x, \cdot)\right)$ is often referred to as the Lévy seed at $x$ of the classical Lévy basis $N=\{N(E) \mid E \in \mathcal{E}\}$ corresponding to $M$ as in Theorem 4.1. By analogy we refer to $\nu_{x}$ as the (free) Lévy seed of $M$ at $x$. Proposition 4.3 then asserts that the distribution of the free Lévy basis $M$ is uniquely determined by the family $\left\{\nu_{x} \mid x \in X\right\}$ of Lévy seeds and the control measure $\kappa$. Accordingly we refer to the quadruplet $\left(\theta, \sigma^{2}, \rho, \kappa\right)$ as the free characteristic quadruplet of $M$. We note further, that since $\Lambda\left(\mu_{x}\right)=\nu_{x}$ for all $x$ in $X$, it is apparent that the one-to-one correspondence in Theorem 4.1 really takes place at the infinitesimal level, i.e. by applying $\Lambda$ at the level of the "infinitesimal seeds".

We consider next a fundamental class of examples of free Lévy bases, namely the so-called factorizable free Lévy bases.

Examples 4.5. (1) Let $\nu$ be a measure from $\mathcal{I D}(\boxplus) \backslash\left\{\delta_{0}\right\}$ with free characteristic triplet $(a, b, r)$. Let further $X$ be a non-empty set equipped with a $\delta$-ring $\mathcal{E}$, and let $\eta: \mathcal{E} \rightarrow[0, \infty]$ be a measure on $\mathcal{E}$. Finally put $\mathcal{E}_{0}=\{E \in \mathcal{E} \mid \eta(E)<\infty\}$, and note that $\mathcal{E}_{0}$ is again a $\delta$-ring. We assume that $\mathcal{E}_{0}$ satisfies condition (2.2). This implies in particular that $\sigma\left(\mathcal{E}_{0}\right)=\sigma(\mathcal{E})$, and that $\eta$ extends uniquely to a $\sigma$-finite measure on $\sigma(\mathcal{E})$.

For each $E$ in $\mathcal{E}_{0}$ we denote by $\nu(E, \cdot)$ the measure in $\mathcal{J D}(\boxplus)$ with free characteristic triplet

$$
\eta(E) \cdot(a, b, r):=(\eta(E) a, \eta(E) b, \eta(E) r) .
$$


If $E_{1}, \ldots, E_{n}$ are disjoint sets from $\mathcal{E}_{0}$, then $\nu\left(E_{1}, \cdot\right) \boxplus \cdots \boxplus \nu\left(E_{n}, \cdot\right)$ has free characteristic triplet

$$
\left(\sum_{k=1}^{n} \eta\left(E_{k}\right)\right) \cdot(a, b, r)=\eta\left(\bigcup_{k=1}^{n} E_{k}\right) \cdot(a, b, r),
$$

and thus equals $\nu\left(\bigcup_{k=1}^{n} E_{k}, \cdot\right)$. Hence by Theorem 3.3 there exists a free Lévy basis $M_{(\eta, \nu)}=\left\{M_{(\eta, \nu)}(E) \mid E \in \mathcal{E}_{0}\right\}$ such that $L^{\mathrm{sp}}\left\{M_{(\eta, \nu)}(E)\right\}=\nu(E, \cdot)$ for all $E$ in $\mathcal{E}_{0}$. It is straightforward to check that in the considered set-up, the triplet $(\Theta, \Sigma, F)$ for $M_{(\eta, \nu)}$ described in Proposition 4.2 is given by

$$
\Theta=a \eta, \quad \Sigma=b \eta \quad \text { and } \quad F=\eta \otimes r,
$$

and consequently the control measure $\kappa$ is given by

$$
\kappa(E)=|a| \eta(E)+b \eta(E)+\eta(E) \int_{\mathbb{R}} \min \left\{1, t^{2}\right\} r(\mathrm{~d} t)=c_{\nu} \eta(E), \quad(E \in \sigma(\mathcal{E})),
$$

where the constant $c_{\nu}$ is given by

$$
c_{\nu}=|a|+b+\int_{\mathbb{R}} \min \left\{1, t^{2}\right\} r(\mathrm{~d} t) .
$$

Note that $c_{\nu}>0$, since $\nu \neq \delta_{0}$. The Lévy seed $\nu_{x}$ at a point $x$ in $X$ (cf. Remark 4.4) consequently has free characteristic triplet $\left(\theta(x), \sigma^{2}(x), \rho(x, \cdot)\right)$ given by

$$
\theta(x)=c_{\nu}^{-1} a, \quad \sigma^{2}(x)=c_{\nu}^{-1} b, \quad \text { and } \quad \rho(x, \cdot)=c_{\nu}^{-1} r .
$$

In particular the Lévy seed $\nu_{x}$ does not depend on $x$. We refer to free Lévy bases in this form as factorizable free Lévy bases.

(2) In the special case where the measure $\nu$ considered in (1) is the standard semi-circle distribution $\frac{1}{2 \pi} \sqrt{4-x^{2}} 1_{[-2,2]}(x) \mathrm{d} x$ we replace the notation $M_{(\eta, \nu)}$ by $G_{\eta}$ and refer to $G_{\eta}$ as a semi-circular Lévy bases. As the free characteristic triplet $(a, b, r)$ of $\nu$ is $(0,1,0)$ in this case, the free cumulant transform of $G_{\eta}(E)$ is given by $\mathcal{C}_{G_{\eta}(E)}(z)=z^{2} \eta(E)$ for all $E$ in $\mathcal{E}_{0}$. In other words $G_{\eta}(E)$ has the semi-circle distribution

$$
\frac{1}{2 \pi \eta(E)} \sqrt{4 \eta(E)-t^{2}} 1_{\left[-2 \eta(E)^{1 / 2}, 2 \eta(E)^{1 / 2}\right]}(t) \mathrm{d} t .
$$

In particular $G_{\eta}(E)$ is a bounded operator for all $E$ in $\mathcal{E}_{0}$. The triplet $(\Theta, \Sigma, F)$ equals $(0, \eta, 0)$, and the constant $c_{\nu}$ in (4.8) equals 1 , so the characteristic quadruplet is $(0,1,0, \eta)$. For each $x$ in $X$ the Lévy seed $\nu_{x}$ is simply $\nu$ itself.

(3) In the special case where the measure $\nu$ considered in (1) is the free Poisson distribution $\frac{1}{2 \pi t} \sqrt{t(4-t)} 1_{[0,4]}(t) \mathrm{d} t$ with parameter 1 , we replace the notation $M_{(\eta, \nu)}$ by $P_{\eta}$.

As the free characteristic triplet $(a, b, r)$ of $\nu$ is $\left(1,0, \delta_{1}\right)$ in this case, the free cumulant transform of $P_{\eta}(E)$ is given by

$$
\mathcal{C}_{P_{\eta}(E)}(z)=\eta(E) z+\eta(E)\left(\frac{1}{1-z}-1-z\right)=\eta(E)\left(\frac{1}{1-z}-1\right), \quad\left(z \in \mathbb{C}^{-}\right),
$$

for all $E$ in $\varepsilon_{0}$. In other words (see e.g. [21, page 35]) the spectral distribution of $P_{\eta}(E)$ is the free Poisson distribution Poiss ${ }^{\boxplus}(\eta(E))$ with parameter $\eta(E)$ given by

$$
\operatorname{Poiss}^{\boxplus}(\eta(E))(\mathrm{d} t)= \begin{cases}(1-\eta(E)) \delta_{0}+\frac{1}{2 \pi t} \sqrt{(t-s)(u-t)} 1_{[s, u]}(t) \mathrm{d} t, & \text { if } \eta(E) \leq 1, \\ \frac{1}{2 \pi t} \sqrt{(t-s)(u-t)} 1_{[s, u]}(t) \mathrm{d} t, & \text { if } \eta(E)>1,\end{cases}
$$

where $s=(1-\eta(E))^{2}$ and $u=(1+\eta(E))^{2}$. In particular $P_{\eta}(E)$ is a bounded operator for all $E$ in $\mathcal{E}_{0}$. The triplet $(\Theta, \Sigma, F)$ equals $\left(\eta, 0, \eta \otimes \delta_{1}\right)$, and the constant $c_{\nu}$ in (4.8) equals 
2 , so that the characteristic quadruplet is $\left(\frac{1}{2}, 0, \frac{1}{2} \delta_{1}, 2 \eta\right)$. For each $x$ in $X$ the Lévy seed $\nu_{x}$ is the Poisson distribution with parameter $\frac{1}{2}$.

Free Lévy bases in this form were previously considered under the name free Poisson random measures in [2].

We close this section by stating two propositions, both of which describe natural and useful constructions with free Lévy bases. As the proofs of these propositions are rather simple, we leave them as exercises for the interested reader.

Proposition 4.6. Let $M=\{M(E) \mid E \in \mathcal{E}\}$ be a free Lévy basis with free characteristic triplet $(\Theta, \Sigma, F)$. Let further $\varphi: X \rightarrow Y$ be mapping from $X$ into a non-empty set $Y$, and define

$$
\mathcal{F}^{0}=\left\{H \subseteq Y \mid \varphi^{-1}(H) \in \mathcal{E}\right\}
$$

and

$$
M \circ \varphi^{-1}=\left\{M\left(\varphi^{-1}(H)\right) \mid H \in \mathcal{F}^{0}\right\} .
$$

Then $\mathcal{F}^{0}$ is a $\delta$-ring and $M \circ \varphi^{-1}$ is a free Lévy basis. If $\mathcal{F}^{0}$ satisfies (2.2), then the free characteristic triplet of $M \circ \varphi^{-1}$ is given by $\left(\Theta \circ \varphi^{-1}, \Sigma \circ \varphi^{-1}, F \circ\left(\varphi, \mathrm{id}_{\mathbb{R}}\right)^{-1}\right)$, where $\mathrm{id}_{\mathbb{R}}$ denotes the identity function on $\mathbb{R}$ and $\left(\varphi, \mathrm{id}_{\mathbb{R}}\right): X \times \mathbb{R} \rightarrow Y \times \mathbb{R}$ is the function given by

$$
\left(\varphi, \operatorname{id}_{\mathbb{R}}\right)(x, t)=(\varphi(x), t) \quad(x \in X, t \in \mathbb{R}) .
$$

Proposition 4.7. Let $M=\{M(E) \mid E \in \mathcal{E}\}$ be a free Lévy basis with free characteristic triplet $(\Theta, \Sigma, F)$. Let further $A$ be a fixed set from $\sigma(\mathcal{E})$, and define

$$
\mathcal{E}^{A}=\{E \in \sigma(\mathcal{E}) \mid A \cap E \in \mathcal{E}\},
$$

and

$$
M^{A}(E)=M(A \cap E), \quad\left(E \in \mathcal{E}^{A}\right) .
$$

Then $M^{A}$ is a new free Lévy basis on $(X, \mathcal{E})$ with free characteristic triplet $\left(\Theta^{A}, \Sigma^{A}, F^{A}\right)$ given by

$$
\Theta^{A}(E)=\Theta(A \cap E), \quad \Sigma^{A}(E)=\Sigma(A \cap E), \quad \text { and } \quad F^{A}(E \times B)=F((A \cap E) \times B)
$$

for any $E$ from $\mathcal{E}$ and any Borel subset $B$ of $\mathbb{R}$.

\section{Integration with respect to free Lévy Bases}

In this section we develop a theory of integration with respect to free Lévy bases in parallel to the corresponding theory for classical Lévy bases in [16]. Throughout the section we consider a $\delta$-ring in a non-empty set $X$, and we assume condition (2.2). We consider further a free Lévy basis $\{M(E) \mid E \in \mathcal{E}\}$ affiliated with a $W^{*}$-probability space $(\mathcal{M}, \tau)$. Now let $s: X \rightarrow \mathbb{R}$ be a simple $\mathcal{E}$-measurable function in the form:

$$
s=\sum_{k=1}^{n} \alpha_{k} 1_{A_{k}}, \quad \text { where } \alpha_{1}, \ldots, \alpha_{n} \in \mathbb{R} \text {, and } A_{1}, \ldots, A_{n} \text { are disjoint sets from } \mathcal{E} .
$$

Then for any $A$ in $\sigma(\mathcal{E})$ we define the integral $\int_{A} s \mathrm{~d} M$ of $s$ over $A$ with respect to $M$ by the formula:

$$
\int_{A} s \mathrm{~d} M=\sum_{k=1}^{n} \alpha_{k} M\left(A_{k} \cap A\right) .
$$

Remarks 5.1. (1) The right hand side of (5.2) is well-defined, since $A_{k} \cap A \in \mathcal{E}$ for all $k$ (cf. (2.1)). Denote by $\operatorname{s\mathcal {N}}(\mathcal{E})$ the class of functions in the form (5.1). Since $\mathcal{E}$ is in particular stable under finite intersections, it follows from the definition of a free Lévy 
basis and standard argumentation that $\mathcal{S} \mathcal{N}(\mathcal{E})$ is a vector space, that the right hand side of (5.2) does not depend on the choice of the representation (5.1) and that

$$
\int_{A}\left(\alpha s+s^{\prime}\right) \mathrm{d} M=\alpha \int_{A} s \mathrm{~d} M+\int_{A} s^{\prime} \mathrm{d} M
$$

for any $s, s^{\prime}$ in $\operatorname{S\mathcal {N}}(\mathcal{E})$, any $A$ in $\sigma(\mathcal{E})$ and any $\alpha$ in $\mathbb{R}$. The definition of a free Lévy basis further entails that $L^{\mathrm{sp}}\left\{\int_{A} s \mathrm{~d} M\right\} \in \mathcal{J D}(\boxplus)$ for any $s$ in $\mathcal{S M}(\mathcal{E})$ and $A$ in $\sigma(\mathcal{E})$.

(2) Let $N=\{N(E) \mid E \in \mathcal{E}\}$ be a classical Lévy basis corresponding to $M$ as in Theorem 4.1. Then for any $s$ in $\operatorname{S\mathcal {N}}(\mathcal{E})$ and $A$ in $\sigma(\mathcal{E})$ the integral $\int_{A} s \mathrm{~d} N$ is defined in [16] exactly as above (with $M$ replaced by $N$ ). It follows then from the algebraic properties of $\Lambda$ that

$$
\Lambda\left(L\left\{\int_{A} s \mathrm{~d} N\right\}\right)=L^{\mathrm{sp}}\left\{\int_{A} s \mathrm{~d} M\right\} .
$$

In parallel to [16] we define next the class $\mathcal{L}^{1}(M)$ of real valued functions on $X$ that are integrable with respect to $M$.

Definition 5.2. Let $f: X \rightarrow \mathbb{R}$ be a $\sigma(\mathcal{E})-\mathcal{B}(\mathbb{R})$-measurable function. Then $f$ is called $M$-integrable, if there exists a sequence $\left(s_{n}\right)_{n \in \mathbb{N}}$ from $\mathcal{S} \mathcal{M}(\mathcal{E})$ such that the following two conditions are satisfied:

(a) $\lim _{n \rightarrow \infty} s_{n}=f$ almost everywhere with respect to the control measure for $M$.

(b) For any $A$ in $\sigma(\mathcal{E})$, the sequence $\left(\int_{A} s_{n} \mathrm{~d} M\right)_{n \in \mathbb{N}}$ converges in the measure topology on $(\mathcal{M}, \tau)$.

The class of $M$-integrable functions $f: X \rightarrow \mathbb{R}$ is denoted by $\mathcal{L}^{1}(M)$.

For a classical Lévy basis $N=\{N(E) \mid E \in \mathcal{E}\}$ the class of $N$-integrable functions, here denoted by $\mathcal{L}^{1}(N)$, was introduced in [16] exactly as in Definition 5.2, but with $M$ replaced by $N$ and convergence in the measure topology replaced by convergence in probability.

For an $M$-integrable function $f$ it is natural to define the integral $\int_{A} f \mathrm{~d} M$ with respect to $M$ as the limit of the sequence appearing in Definition 5.2(b). We proceed next to show that this limit does not depend on the choice of approximating sequence satisfying conditions (a) and (b) from the afore mentioned definition, while simultaneously establishing that $\mathcal{L}^{1}(M)=\mathcal{L}^{1}(N)$, if $N$ is the classical Lévy basis corresponding to $M$ as in Theorem 4.1.

Proposition 5.3. Let $N=\{N(E) \mid E \in \mathcal{E}\}$ be a classical Lévy basis corresponding to $M$ as in Theorem 4.1. Then the following assertions hold:

(i) $\mathcal{L}^{1}(M)=\mathcal{L}^{1}(N)$.

(ii) If $f \in \mathcal{L}^{1}(M)$ and $\left(s_{n}\right)_{n \in \mathbb{N}}$ and $\left(t_{n}\right)_{n \in \mathbb{N}}$ are two sequences from $\mathcal{S} \mathcal{M}(\mathcal{E})$, both satisfying conditions (a) and (b) of Definition 5.2, then for any $A$ in $\sigma(\mathcal{E})$ the sequences $\left(\int_{A} s_{n} \mathrm{~d} M\right)_{n \in \mathbb{N}}$ and $\left(\int_{A} t_{n} \mathrm{~d} M\right)$ share the same limit in the measure topology.

Proof. Let $f$ be a function from $\mathcal{L}^{1}(M)$, and let $\left(s_{n}\right)_{n \in \mathbb{N}}$ be a sequence from $\operatorname{S\mathcal {N}}(\mathcal{E})$ satisfying conditions (a) and (b) of Definition 5.2. Then for any $n, m$ in $\mathbb{N}$ it follows from (1) and (2) in Remark 5.1 that

$$
L\left\{\int_{A} s_{n} \mathrm{~d} N-\int_{A} s_{m} \mathrm{~d} N\right\}=\Lambda^{-1}\left(L^{\mathrm{sp}}\left\{\int_{A} s_{n} \mathrm{~d} M-\int_{A} s_{m} \mathrm{~d} M\right\}\right) \longrightarrow \delta_{0}, \quad \text { as } n, m \rightarrow \infty,
$$

so that $\left(\int_{A} s_{n} \mathrm{~d} N\right)_{n \in \mathbb{N}}$ is a Cauchy-sequence in probability and hence convergent in probability. Since $M$ and $N$ have the same control measure, this verifies that $f \in \mathcal{L}^{1}(N)$ 
and hence the inclusion $\mathcal{L}^{1}(M) \subseteq \mathcal{L}^{1}(N)$. The reverse inclusion follows by similar argumentation, applying $\Lambda$ rather than $\Lambda^{-1}$ and using completeness of the measure topology. Hence (i) follows.

Assume next that $\left(t_{n}\right)_{n \in \mathbb{N}}$ is another sequence from $\mathcal{S} \mathcal{M}(\mathcal{E})$ satisfying conditions (a) and (b) of Definition 5.2. The same argumentation as above then shows that the sequence $\left(\int_{A} t_{n} \mathrm{~d} N\right)_{n \in \mathbb{N}}$ converges in probability as well for any $A$ in $\sigma(\mathcal{E})$, and it follows then from [19] that the limit must equal that of $\left(\int_{A} s_{n} \mathrm{~d} N\right)_{n \in \mathbb{N}}$. Therefore the mixed sequence

$$
\int_{A} s_{1} \mathrm{~d} N, \int_{A} t_{1} \mathrm{~d} N, \int_{A} s_{2} \mathrm{~d} N, \int_{A} t_{2} \mathrm{~d} N, \ldots
$$

is also convergent and hence a Cauchy sequence in probability. Arguing as above the properties of $\Lambda$ entail that the sequence

$$
\int_{A} s_{1} \mathrm{~d} M, \int_{A} t_{1} \mathrm{~d} M, \int_{A} s_{2} \mathrm{~d} M, \int_{A} t_{2} \mathrm{~d} M, \ldots
$$

is then a Cauchy sequence and hence convergent in the measure topology. Since the measure topology is Hausdorff, (ii) now follows by sub-sequence considerations.

Definition 5.4. Let $f$ be an $M$-integrable function, and let $\left(s_{n}\right)_{n \in \mathbb{N}}$ be a sequence of functions from $\operatorname{SM}(\mathcal{M})$ satisfying conditions (a) and (b) of Definition 5.2. Then for any $A$ in $\sigma(\mathcal{E})$ the integral $\int_{A} f \mathrm{~d} M$ of $f$ over $A$ with respect to $M$ is defined by

$$
\int_{A} f \mathrm{~d} M=\lim _{n \rightarrow \infty} \int_{A} s_{n} \mathrm{~d} M,
$$

where the limit is in the measure topology.

In the following remark we list next a number of rather immediate properties of the integral introduced in the definition above.

Remarks 5.5. (1) If $f$ is in $\mathcal{L}^{1}(M)$ and $A \in \mathcal{E}$, then $\int_{A} f \mathrm{~d} M$ is a selfadjoint operator affiliated with $(\mathcal{M}, \tau)$ (because $f$ is real-valued and the adjoint operation is continuous in the measure topology).

(2) It follows Proposition 5.3(i) that $\mathcal{L}^{1}(M)$ is a vector space. This also follows directly from Definition 5.2 and the fact that the linear operations on $\overline{\mathcal{M}}$ are continuous in the measure topology. This latter fact together with Remark 5.1(1) further entail that

$$
\int_{A}(\alpha f+g) \mathrm{d} M=\alpha \int_{A} f \mathrm{~d} M+\int_{A} g \mathrm{~d} M
$$

for any $f, g$ in $\mathcal{L}^{1}(M), A$ in $\sigma(\mathcal{E})$ and $\alpha$ in $\mathbb{R}$.

(3) In Remark 5.1(1) we observed that $\int_{A} s \mathrm{~d} M \in \mathcal{I D}(\boxplus)$ for any $s$ in $\mathcal{S} \mathcal{N}(\mathcal{E})$ and $A$ in $\sigma(\mathcal{E})$. Since $\mathcal{J D}(\boxplus)$ is closed under weak convergence, and since convergence in the measure topology implies weak convergence of the spectral distributions (cf. (2.5)), it follows that $\int_{A} f \mathrm{~d} M \in \mathcal{J D}(\boxplus)$ for any $f$ in $\mathcal{L}^{1}(M)$. Moreover, the continuity of $\Lambda$ in combination with Remark 5.1(2) imply that

$$
\Lambda\left(L\left\{\int_{A} f \mathrm{~d} N\right\}\right)=L^{\mathrm{sp}}\left\{\int_{A} f \mathrm{~d} M\right\}
$$

for any $f$ in $\mathcal{L}^{1}(M)=\mathcal{L}^{1}(N)$, where $N$ is a classical Lévy basis corresponding to $M$ as in Theorem 4.1. 
(4) Let $K$ be a positive integer, let $f_{1}, \ldots, f_{K}$ be functions from $\mathcal{L}^{1}(M)$, and let $A_{1}, \ldots, A_{K}$ be disjoint sets from $\sigma(\mathcal{E})$. Then the integrals $\int_{A_{1}} f_{1} \mathrm{~d} M, \ldots, \int_{A_{K}} f_{K} \mathrm{~d} M$ are freely independent operators in $\overline{\mathcal{M}}$. This fact follows immediately from (5.2) and the definition of a free Lévy basis, in case $f_{1}, \ldots, f_{K} \in \mathcal{S} \mathcal{N}(\mathcal{E})$. The extension to general functions in $\mathcal{L}^{1}(M)$ subsequently follows directly from Definition 5.4, since free independence is preserved under limits in the measure topology (see Proposition 5.4 in [2]).

(5) Since $\mathcal{L}^{1}(M)=\mathcal{L}^{1}(N)$ (with $N$ as in (3)), Theorem 2.7 in [16] immediately provides the following characterization of $\mathcal{L}^{1}(M)$ in terms of the characteristic quadruplet $\left(\theta, \sigma^{2}, \rho, \kappa\right)$ of $M: \mathrm{A} \sigma(\mathcal{E})-\mathcal{B}(\mathbb{R})$-measurable function $f: X \rightarrow \mathbb{R}$ belongs to $\mathcal{L}^{1}(M)$, if and only if the following three conditions are satisfied:

(a) $\int_{X}\left|f(x) \theta(x)+\int_{\mathbb{R}}(\varsigma(f(x) t)-f(x) \varsigma(t)) \rho(x, \mathrm{~d} t)\right| \kappa(\mathrm{d} x)<\infty$.

(b) $\int_{X} f(x)^{2} \sigma^{2}(x) \kappa(\mathrm{d} x)<\infty$.

(c) $\int_{X}\left(\int_{\mathbb{R}} \min \left\{1, f(x)^{2} t^{2}\right\} \rho(x, \mathrm{~d} t)\right) \kappa(\mathrm{d} x)<\infty$.

In the affirmative case it follows further from (3) and [16, Theorem 2.7] that the free characteristic triplet $\left(a_{f}, \sigma_{f}^{2}, F_{f}\right)$ of $\int_{X} f \mathrm{~d} M$ is given by

(d) $a_{f}=\int_{X}\left(f(x) \theta(x)+\int_{\mathbb{R}}(\varsigma(f(x) t)-f(x) \varsigma(t)) \rho(x, \mathrm{~d} t)\right) \kappa(\mathrm{d} x)$.

(e) $\sigma_{f}^{2}=\int_{X} f(x)^{2} \sigma^{2}(x) \kappa(\mathrm{d} x)$.

(f) $F_{f}(B)=F(\{(x, t) \in X \times \mathbb{R} \mid f(x) t \in B \backslash\{0\}\})$ for any Borel set $B$ in $\mathbb{R}$.

In (f) $F$ is the measure on $\sigma(\mathcal{E}) \otimes \mathcal{B}(\mathbb{R})$ described in Proposition 4.2 (or equivalently given by (4.7)). For $f$ in $\mathcal{L}^{1}(M)$ the measure $F_{f}$ is a Lévy measure on $\mathbb{R}$, and by e.g. an extension argument it follows that

$$
\int_{\mathbb{R}} g(t) F_{f}(\mathrm{~d} t)=\int_{X \times \mathbb{R}} g(f(x) t) \cdot 1_{\mathbb{R} \backslash\{0\}}(f(x) t) F(\mathrm{~d} x, \mathrm{~d} t),
$$

for any function $g$ in $\mathcal{L}^{1}\left(F_{f}\right)$.

Knowing the free characteristic triplet of $\int_{X} f \mathrm{~d} M$ (as described in Remark 5.5(5)), we can easily derive the following analog of Proposition 2.6 in [16], which generalizes Proposition 4.3 (in the present paper) from indicator functions to general functions in $\mathcal{L}^{1}(M)$.

Corollary 5.6. Let $M=\{M(E) \mid E \in \mathcal{E}\}$ be a free Lévy basis with free characteristic quadruplet $\left(\theta, \sigma^{2}, \rho, \kappa\right)$, and let $f$ be a function from $\mathcal{L}^{1}(M)$. Consider further the kernel $R: X \times \mathbb{C}^{-} \rightarrow \mathbb{C}$ set out in Proposition 4.3. Then the function $x \mapsto R(x, z f(x))$ is in $\mathcal{L}^{1}(\kappa)$ for all $z$ in $\mathbb{C}^{-}$, and the free cumulant transform of $\int_{X} f \mathrm{~d} M$ is given by

$$
\mathcal{C}_{\int_{X} f \mathrm{~d} M}(z)=\int_{X} R(x, f(x) z) \kappa(\mathrm{d} x), \quad\left(z \in \mathbb{C}^{-}\right) .
$$

Recall that for fixed $x$ in $X$ the function $R(x, \cdot)$ is the free cumulant transform of the Lévy seed $\nu_{x}$ at $x$. Thus, from the infinitesimal point of view, (5.4) shows that the distribution of the integral $\int_{X} f \mathrm{~d} M$ is obtained by scaling $\nu_{x}$ by $f(x)$ at each $x$, followed by an averaging with respect to the control measure $\kappa$.

Proof of Corollary 5.6. Consider the free characteristic triplet $\left(a_{f}, \sigma_{f}^{2}, F_{f}\right)$ for $\int_{X} f \mathrm{~d} M$ (given in Remark 5.5(5)). For $z$ in $\mathbb{C}^{-}$it follows then by (5.3) and (4.7) that

$$
\begin{aligned}
\int_{\mathbb{R}}\left(\frac{1}{1-t z}-1-z \varsigma(t)\right) F_{f}(\mathrm{~d} t) & =\int_{X \times \mathbb{R}}\left(\frac{1}{1-t z f(x)}-1-z \varsigma(t f(x))\right) F(\mathrm{~d} x, \mathrm{~d} t) \\
& =\int_{X}\left(\int_{\mathbb{R}}\left(\frac{1}{1-t z f(x)}-1-z \varsigma(t f(x))\right) \rho(x, \mathrm{~d} t)\right) \kappa(\mathrm{d} x),
\end{aligned}
$$


and consequently

$$
\begin{aligned}
& \mathcal{C}_{\int_{X} f \mathrm{~d} M}(z)=z a_{f}+z^{2} \sigma_{f}^{2}+\int_{\mathbb{R}}\left(\frac{1}{1-t z}-1-z \varsigma(t)\right) F_{f}(\mathrm{~d} t) \\
& =\int_{X} z\left(f(x) \theta(x)+\int_{\mathbb{R}}(\varsigma(f(x) t)-f(x) \varsigma(t)) \rho(x, \mathrm{~d} t)\right) \kappa(\mathrm{d} x) \\
& \quad+z^{2} \int_{X} f(x)^{2} \sigma^{2}(x) \kappa(\mathrm{d} x)+\int_{X}\left(\int_{\mathbb{R}}\left(\frac{1}{1-t z f(x)}-1-z \varsigma(t f(x))\right) \rho(x, \mathrm{~d} t)\right) \kappa(\mathrm{d} x), \\
& =\int_{X}\left(z f(x) \theta(x)+z^{2} f(x)^{2} \sigma^{2}(x)+\int_{\mathbb{R}}\left(\frac{1}{1-t z f(x)}-1-z f(x) \varsigma(t)\right) \rho(x, \mathrm{~d} t)\right) \kappa(\mathrm{d} x), \\
& =\int_{X} R(z f(x), x) \kappa(\mathrm{d} x),
\end{aligned}
$$

as desired.

Proposition 5.7. Let $M=\{M(E) \mid E \in \mathcal{E}\}$ be a free Lévy basis with free characteristic triplet $(\Theta, \Sigma, F)$ and quadruplet $\left(\theta, \sigma^{2}, \rho(\cdot, \mathrm{d} t), \kappa\right)$. Let further $f: X \rightarrow \mathbb{R}$ be a $\sigma(\mathcal{E})$-measurable function, and define

$$
\mathcal{E}(f)=\left\{E \in \sigma(\mathcal{E}) \mid f 1_{E} \in \mathcal{L}^{1}(M)\right\},
$$

and

$$
f \cdot M(E)=\int_{X} f 1_{E} \mathrm{~d} M, \quad(E \in \mathcal{E}(f)) .
$$

Then the following statements hold:

(i) The family $\mathcal{E}(f)$ is $\delta$-ring and $f \cdot M=\{f \cdot M(E) \mid E \in \mathcal{E}(f)\}$ is free Lévy basis.

(ii) Suppose $\mathcal{E}(f)$ satisfies (2.2). Then the free characteristic triplet $\left(\Theta^{f}, \Sigma^{f}, F^{f}\right)$ for $f \cdot M$ is given by

(a) $\Theta^{f}(E)=\int_{E}\left(f(x) \theta(x)+\int_{\mathbb{R}}(\varsigma(f(x) t)-f(x) \varsigma(t)) \rho(x, \mathrm{~d} t)\right) \kappa(\mathrm{d} x)$,

(b) $\Sigma^{f}(E)=\int_{E} f(x)^{2} \sigma^{2}(x) \kappa(\mathrm{d} x)$,

(c) $\int_{X \times \mathbb{R}} g(x, t) F^{f}(\mathrm{~d} x, \mathrm{~d} t)=\int_{X \times \mathbb{R}} g(x, t f(x)) 1_{\mathbb{R} \backslash\{0\}}(t f(x)) F(\mathrm{~d} x, \mathrm{~d} t)$,

where (c) holds for any $\sigma(\mathcal{E}(f)) \otimes \mathcal{B}(\mathbb{R})$-measurable function $g: X \times \mathbb{R} \rightarrow[0, \infty)$.

Proof. (i) According to Remark 5.5(5) a set $E$ from $\sigma(\mathcal{E})$ belongs to $\mathcal{E}(f)$, if and only if the following three conditions are satisfied:

- $\int_{E}\left|f(x) \theta(x)+\int_{\mathbb{R}}(\varsigma(f(x) t)-f(x) \varsigma(t)) \rho(x, \mathrm{~d} t)\right| \kappa(\mathrm{d} x)<\infty$.

- $\int_{E} f(x)^{2} \sigma^{2}(x) \kappa(\mathrm{d} x)<\infty$.

- $\int_{E}\left(\int_{\mathbb{R}} \min \left\{1, f(x)^{2} t^{2}\right\} \rho(x, \mathrm{~d} t)\right) \kappa(\mathrm{d} x)<\infty$.

In particular it is apparent that $\mathcal{E}(f)$ is a $\delta$-ring with the hereditary property that the conditions $E_{1} \in \sigma(\mathcal{E}), E_{2} \in \mathcal{E}(f)$ and $E_{1} \subseteq E_{2}$ imply that $E_{1} \in \mathcal{E}(f)$.

The fact that $f \cdot M$ satisfies conditions (a),(b) and (d) in Definition 3.1 follows from, respectively, (4), (2) and (3) of Remark 5.5. To verify condition (c) in Definition 3.1 consider a decreasing sequence $\left(E_{n}\right)_{n \in \mathbb{N}}$ of sets from $\mathcal{E}(f)$, such that $E_{n} \downarrow \emptyset$ as $n \rightarrow \infty$. It follows then from Corollary 5.6 that for any $z$ in $\mathbb{C}^{-}$the function $x \mapsto R\left(x, z f(x) 1_{E_{1}}(x)\right)=$ $R(x, z f(x)) 1_{E_{1}}(x)$ is in $\mathcal{L}^{1}(\kappa)$, and hence by dominated convergence

$$
\mathcal{C}_{\int_{X} f 1_{E_{n}} \mathrm{~d} M}(z)=\int_{E_{n}} R(x, z f(x)) 1_{E_{1}}(x) \kappa(\mathrm{d} x) \underset{n \rightarrow \infty}{\longrightarrow} 0,
$$

for any $z$ in $\mathbb{C}^{-}$. By Proposition 2.2 this implies that $L^{\mathrm{sp}}\left\{\int_{X} f 1_{E_{n}} \mathrm{~d} M\right\} \stackrel{\mathrm{w}}{\rightarrow} \delta_{0}$, i.e. $\int_{X} f 1_{E_{n}} \mathrm{~d} M \rightarrow 0$ in the measure topology (cf. (2.5)). 
(ii) The formulae for $\Theta^{f}$ and $\Sigma^{f}$ follow readily from Remark 5.5(5), which also yields that

$$
F^{f}(E \times B)=F\left(\left\{(x, t) \in X \times \mathbb{R} \mid 1_{E}(x) f(x) t \in B \backslash\{0\}\right\}\right)
$$

for any $E$ in $\mathcal{E}(f)$ and any Borel set $B$ in $\mathbb{R}$. From this condition the measure $F^{f}$ on $\sigma(\mathcal{E}(f)) \otimes \mathcal{B}(\mathbb{R})$ may be identified as the concentration to $X \times(\mathbb{R} \backslash\{0\})$ of the transformation of $F$ by the mapping $\Psi: X \times \mathbb{R} \rightarrow X \times \mathbb{R}$ given by $\Psi(x, t)=(x, t f(x))$. Specifically this means that

$$
F^{f}(C)=F\left(\Psi^{-1}(C \cap(X \times(\mathbb{R} \backslash\{0\})))\right)=\int_{X \times \mathbb{R}} 1_{C}(x, t f(x)) 1_{\mathbb{R} \backslash\{0\}}(t f(x)) F(\mathrm{~d} x, \mathrm{~d} t),
$$

for any set $C$ in $\sigma(\mathcal{E}(f)) \otimes \mathcal{B}(\mathbb{R})$, which is in accordance with the formula for $F^{f}(E \times B)=$ $F_{E}^{f}(B)$ given above. By e.g. an extension argument it follows further that

$$
\int_{X \times \mathbb{R}} g(x, t) F^{f}(\mathrm{~d} x, \mathrm{~d} t)=\int_{X \times \mathbb{R}} g(x, t f(x)) 1_{\mathbb{R} \backslash\{0\}}(t f(x)) F(\mathrm{~d} x, \mathrm{~d} t)
$$

for any positive and measurable function $g$.

\section{The Lévy-Itô decomposition for free Lévy bases}

Throughout this section we consider a non-empty set $X$ equipped with a $\delta$-ring $\mathcal{E}$, which contains a sequence $\left(E_{n}\right)_{n \in \mathbb{N}}$ of sets such that $X=\bigcup_{n \in \mathbb{N}} E_{n}$. We consider further a free Lévy basis $M=\{M(E) \mid E \in \mathcal{E}\}$ with free characteristic triplet $(\Theta, \Sigma, F)$ (in particular $F$ is a $\sigma$-finite measure on $\sigma(\mathcal{E}) \otimes \mathcal{B}(\mathbb{R})$ ). Our objective is to establish a Lévy-Itô type representation of $M$. For this we introduce first a free Poisson random measure $P_{F}=\left\{P_{F}(A) \mid A \in \mathcal{A}_{F}\right\}$ on $X \times \mathbb{R}$ affiliated with some $W^{*}$-probability space $(\mathcal{M}, \tau)$, and where

$$
\mathcal{A}_{F}=\{A \in \sigma(\mathcal{E}) \otimes \mathcal{B}(\mathbb{R}) \mid F(A)<\infty\},
$$

and

$$
L^{\mathrm{sp}}\left\{P_{F}(A)\right\}=\text { Poiss }^{\boxplus}(F(A)) \text { for all } A \text { in } \mathcal{A}_{F} .
$$

We note in particular that $\sigma\left(\mathcal{A}_{F}\right)=\sigma(\mathcal{E}) \otimes \mathcal{B}(\mathbb{R})$, since $F$ is $\sigma$-finite. We recall also (cf. Example 4.5(3)) that the free characteristic triplet and quadruplet of $P_{F}$ are $\left(F, 0, F \otimes \delta_{1}\right)$ and $\left(\frac{1}{2}, 0, \frac{1}{2} \delta_{1}, 2 F\right)$, respectively.

Consider now additionally the function $h: X \times \mathbb{R} \rightarrow \mathbb{R}$ given by

$$
h(x, t)=t, \quad(x \in X, t \in \mathbb{R}) .
$$

By application of Proposition 5.7 we may then consider yet another free Lévy basis $h \cdot P_{F}=\left\{h \cdot P_{F}(A) \mid A \in \mathcal{A}_{F}(h)\right\}$ on $X \times \mathbb{R}$, where

$$
\mathcal{A}_{F}(h)=\left\{A \in \sigma(\mathcal{E}) \otimes \mathcal{B}(\mathbb{R}) \mid h 1_{A} \in \mathcal{L}^{1}\left(P_{F}\right)\right\},
$$

and

$$
h \cdot P_{F}(A)=\int_{X \times \mathbb{R}} 1_{A} h \mathrm{~d} P_{F}=\int_{X \times \mathbb{R}} 1_{A}(x, t) t P_{F}(\mathrm{~d} x, \mathrm{~d} t), \quad(A \in \mathcal{A}(h)) .
$$

Lemma 6.1. In the framework set up above the following assertions hold:

(i) For any positive number $\epsilon$ we have that

$$
\{E \times(\mathbb{R} \backslash[-\epsilon, \epsilon]) \mid E \in \mathcal{E}\} \subseteq \mathcal{A}_{F}(h) .
$$

In particular $\mathcal{A}_{F}(h)$ satisfies (2.2). 
(ii) The free characteristic triplet for $h \cdot P_{F}$ is given by $\left(F^{h}, 0,\left(F \otimes \delta_{1}\right)^{h}\right)$, where

$$
\begin{aligned}
F^{h}(A) & =\int_{A} \varsigma(t) F(\mathrm{~d} x, \mathrm{~d} t), \quad\left(A \in \mathcal{A}_{F}(h)\right), \\
\left(F \otimes \delta_{1}\right)^{h}(A \times B) & =F(A \cap(X \times(B \backslash\{0\}))), \quad(A \in \sigma(\mathcal{E}) \otimes \mathcal{B}(\mathbb{R}), B \in \mathcal{B}(\mathbb{R})) .
\end{aligned}
$$

(iii) If $E \in \mathcal{E}$ such that $\int_{-1}^{1}|t| F_{E}(\mathrm{~d} t)<\infty$, then $E \times \mathbb{R} \in \mathcal{A}_{F}(h)$.

Proof. (i) Let $E$ from $\mathcal{E}$ be given. Clearly $E \times(\mathbb{R} \backslash[-\epsilon, \epsilon]) \in \sigma(\mathcal{E}) \otimes \mathcal{B}(\mathbb{R})=\sigma\left(\mathcal{A}_{F}\right)$. It remains thus to verify that $h 1_{E \times(\mathbb{R} \backslash[-\epsilon, \epsilon])} \in \mathcal{L}^{1}\left(P_{F}\right)$. Recalling from Example 4.5(3) that the free characteristic quadruplet for $P_{F}$ is $\left(\frac{1}{2}, 0, \frac{1}{2} \delta_{1}, 2 F\right)$, it follows by a straightforward application of Remark 5.5(5) that this amounts to the conditions:

$$
\infty>\int_{E \times(\mathbb{R} \backslash[-\epsilon, \epsilon])}|\varsigma(h(x, t))| F(\mathrm{~d} x, \mathrm{~d} t)=\int_{\mathbb{R} \backslash[-\epsilon, \epsilon]}|\varsigma(t)| F_{E}(\mathrm{~d} t),
$$

and

$$
\infty>\int_{E \times(\mathbb{R} \backslash[-\epsilon, \epsilon])} \min \left\{1, h(x, t)^{2}\right\} F(\mathrm{~d} x, \mathrm{~d} t)=\int_{\mathbb{R} \backslash[-\epsilon, \epsilon]} \min \left\{1, t^{2}\right\} F_{E}(\mathrm{~d} t) .
$$

Both conditions are satisfied, since $F_{E}$ is a Lévy measure.

(ii) Since the free characteristic quadruplet for $P_{F}$ is $\left(\frac{1}{2}, 0, \frac{1}{2} \delta_{1}, 2 F\right)$ it follows readily from Proposition 5.7 that the free characteristic triplet for $h \cdot P_{F}$ is given as $\left(F^{h}, 0,\left(F \times \delta_{1}\right)^{h}\right)$, where

$$
\begin{aligned}
F^{h}(A) & =\int_{A}\left(\frac{1}{2} h(x, t)+\int_{\mathbb{R}}(\varsigma(h(x, t) s)-h(x, t) \varsigma(s)) \frac{1}{2} \delta_{1}(\mathrm{~d} s)\right) 2 F(\mathrm{~d} x, \mathrm{~d} t) \\
& =\int_{A}(t+\varsigma(t)-t) F(\mathrm{~d} x, \mathrm{~d} t)=\int_{A} \varsigma(t) F(\mathrm{~d} x, \mathrm{~d} t)
\end{aligned}
$$

for any $A$ in $\mathcal{A}_{F}(h)$. For $A$ in $\sigma(\mathcal{E}) \otimes \mathcal{B}(\mathbb{R})$ and $B$ in $\mathcal{B}(\mathbb{R})$ Proposition 5.7 further yields that

$$
\begin{aligned}
\left(F \otimes \delta_{1}\right)^{h}(A \times B) & =\int_{X \times \mathbb{R} \times \mathbb{R}} 1_{A}(x, t) 1_{B}(\operatorname{sh}(x, t)) 1_{\mathbb{R} \backslash\{0\}}(\operatorname{sh}(x, t)) F \otimes \delta_{1}(\mathrm{~d} x, \mathrm{~d} t, \mathrm{~d} s) \\
& =\int_{X \times \mathbb{R}} 1_{A}(x, t) 1_{B \backslash\{0\}}(t) F(\mathrm{~d} x, \mathrm{~d} t)=F(A \cap(X \times(B \backslash\{0\}))) .
\end{aligned}
$$

(iii) Assume that $E \in \mathcal{E}$ such that $\int_{-1}^{1}|t| F_{E}(\mathrm{~d} t)<\infty$. We must verify that $h 1_{E \times \mathbb{R}} \in$ $\mathcal{L}^{1}\left(P_{F}\right)$, and as in the proof of (ii) this amounts to the conditions:

$$
\infty>\int_{\mathbb{R}}|\varsigma(t)| F_{E}(\mathrm{~d} t)=F_{E}(\mathbb{R} \backslash[-1,1])+\int_{-1}^{1}|t| F_{E}(\mathrm{~d} t),
$$

and

$$
\infty>\int_{\mathbb{R}} \min \left\{1, t^{2}\right\} F_{E}(\mathrm{~d} t),
$$

which are clearly satisfied by the assumption on $E$ and since $F_{E}$ is a Lévy measure.

Proposition 6.2. Consider the framework set up in the beginning of this section, and assume that $\int_{-1}^{1}|\varsigma(t)| F_{E}(\mathrm{~d} t)<\infty$ for all $E$ in $\mathcal{E}$. Assume further that there exists a free semi-circular Lévy basis $G_{\Sigma}=\left\{G_{\Sigma}(E) \mid E \in \mathcal{E}\right\}$ in $(\mathcal{M}, \tau)$ with free characteristic triplet $(0, \Sigma, 0)$, which is freely independent of $P_{F}$. For each $E$ in $\mathcal{E}$ put

$$
\tilde{M}(E)=\left(\Theta(E)-\int_{E \times \mathbb{R}} \varsigma(t) F(\mathrm{~d} x, \mathrm{~d} t)\right) \mathbf{1}_{\mathcal{M}}+G_{\Sigma}(E)+h \cdot P_{F}(E \times \mathbb{R})
$$

where $\mathbf{1}_{\mathcal{M}}$ denotes the unit of $\mathcal{M}$.

Then $\tilde{M}$ is a free Lévy basis, and for any $E$ in $\mathcal{E}$ the selfadjoint operators $M(E)$ and $\tilde{M}(E)$ share the same spectral distribution. 
Before the proof we note that the assumed existence of $G_{\Sigma}$ which is freely independent of $P_{F}$ can always be realized by replacing $(\mathcal{M}, \tau)$ by its free product with another $W^{*}$ probability space $\left(\mathcal{N}^{\prime}, \tau^{\prime}\right)$ which contains a free semicircular basis with the specified characteristic triplet. In comparison with the classical Lévy-Itô Decomposition we note also that

by definition of $h \cdot P_{F}$.

$$
h \cdot P_{F}(E \times \mathbb{R})=\int_{E \times \mathbb{R}} t P_{F}(\mathrm{~d} x, \mathrm{~d} t),
$$

Proof. For each $E$ in $\mathcal{E}$ denote by $M_{1}(E), M_{2}(E)$ and $M_{3}(E)$ the three terms on the right hand side of (6.1) (in order of appearance). Note in particular that $M_{3}(E)$ is well-defined according to Lemma 6.1(iii). Clearly $M_{1}$ and $M_{2}$ are free Lévy bases on $(X, \mathcal{E})$ with free characteristic triplets $(\Pi, 0,0)$ and $(0, \Sigma, 0)$, respectively, where we have introduced the signed measure

$$
\Pi(E)=\Theta(E)-\int_{E \times \mathbb{R}} \varsigma(t) F(\mathrm{~d} x, \mathrm{~d} t), \quad(E \in \mathcal{E}) .
$$

Considering the mapping $\psi: X \times \mathbb{R} \rightarrow X$ given by $\psi(x, t)=x$ for all $(x, t)$ in $X \times \mathbb{R}$, we note next that $M_{3}(E)=h \cdot P_{F}\left(\psi^{-1}(E)\right)$ for all $E$ in $\mathcal{E}$, and therefore Proposition 4.6 yields that $M_{3}$ is a free Lévy basis with free characteristic triplet $\left(F^{h} \circ \psi^{-1}, 0,\left(F \otimes \delta_{1}\right)^{h} \circ\left(\psi, \mathrm{id}_{\mathbb{R}}\right)^{-1}\right)$, where $F^{h}$ and $\left(F \otimes \delta_{1}\right)^{h}$ are as set out in Lemma 6.1(ii).

Since $M_{1}, M_{2}, M_{3}$ are freely independent, their sum, $\tilde{M}$, is again a free Lévy basis with free characteristic triplet $\left(\Pi+F^{h} \circ \psi^{-1}, \Sigma,\left(F \otimes \delta_{1}\right)^{h} \circ\left(\psi, \mathrm{id}_{\mathbb{R}}\right)^{-1}\right)$. For any set $E$ from $\mathcal{E}$ note here that by Lemma 6.1(ii)

$$
\Pi(E)+F^{h} \circ \psi^{-1}(E)=\Theta(E)-\int_{E \times \mathbb{R}} \varsigma(t) F(\mathrm{~d} x, \mathrm{~d} t)+F^{h}(E \times \mathbb{R})=\Theta(E),
$$

and for any Borel subset $B$ of $\mathbb{R}$

$$
\begin{aligned}
\left(F \otimes \delta_{1}\right)^{h} \circ\left(\psi, \operatorname{id}_{\mathbb{R}}\right)^{-1}(E \times B) & =\left(F \otimes \delta_{1}\right)^{h}(E \times \mathbb{R} \times B)=F((E \times \mathbb{R}) \cap(X \times(B \backslash\{0\}))) \\
=F(E \times(B \backslash\{0\})) & =F_{E}(B \backslash\{0\})=F_{E}(B)=F(E \times B),
\end{aligned}
$$

where we have used that $F_{E}(\{0\})=0$. Since the last equation above determines $F$ uniquely on $\sigma(\mathcal{E}) \otimes \mathcal{B}(\mathbb{R})$, we conclude that $\left(F \otimes \delta_{1}\right)^{h} \circ\left(\psi, \operatorname{id}_{\mathbb{R}}\right)^{-1}=F$. Altogether $\tilde{M}$ has the same free characteristic triplet as $M$, which clearly implies that $L^{\mathrm{sp}}(\tilde{M}(E))=L^{\mathrm{sp}}(M(E))$ for all $E$ in $\mathcal{E}$.

We return now to the general framework set up in the beginning of this section without imposing the condition $\int_{\mathbb{R}}|\varsigma(t)| F_{E}(\mathrm{~d} t)<\infty$. For each $\epsilon$ in $(0, \infty)$ we then define $M^{(\epsilon)}=\left\{M^{(\epsilon)}(E) \mid E \in \mathcal{E}\right\}$ by

$$
M^{(\epsilon)}(E)=h \cdot P_{F}(E \times(\mathbb{R} \backslash[-\epsilon, \epsilon]))-\left(\int_{\mathbb{R} \backslash[-\epsilon, \epsilon]} \varsigma(t) F_{E}(\mathrm{~d} t)\right) \mathbf{1}_{\mathcal{M}}, \quad(E \in \mathcal{E}) .
$$

Lemma 6.1(i) guarantees that $M^{(\epsilon)}$ is well-defined. With $\psi$ as in the proof of Proposition 6.2 note that $h \cdot P_{f}(E \times(\mathbb{R} \backslash[-\epsilon, \epsilon]))=h \cdot P_{f}\left(X \times(\mathbb{R} \backslash[-\epsilon, \epsilon]) \cap \psi^{-1}(E)\right)$. Hence Propositions 4.6-4.7 in combination with Lemma 6.1(ii) entail that $M^{(\epsilon)}$ is a free Lévy basis with free characteristic triplet $\left(0,0, F^{(\epsilon)}\right)$, where

$$
\begin{aligned}
F^{(\epsilon)}(E \times B) & =\left(F \otimes \delta_{1}\right)^{h}\left((X \times(\mathbb{R} \backslash[-\epsilon, \epsilon]) \times \mathbb{R}) \cap\left(\left(\psi, \operatorname{id}_{\mathbb{R}}\right)^{-1}(E \times B)\right)\right) \\
& =F((X \times(\mathbb{R} \backslash[-\epsilon, \epsilon])) \cap(E \times \mathbb{R}) \cap(X \times(B \backslash\{0\}))) \\
& =F(E \times(B \backslash[-\epsilon, \epsilon])) \\
& =F_{E}(B \backslash[-\epsilon, \epsilon])
\end{aligned}
$$

for any $E$ in $\mathcal{E}$ and $B$ in $\mathcal{B}(\mathbb{R})$. 
Lemma 6.3. (i) With $M^{(\epsilon)}$ as defined in (6.2), the sequence $\left(M^{(1 / n)}(E)\right)_{n \in \mathbb{N}}$ is convergent in the measure topology for any $E$ in $\mathcal{E}$.

(ii) If we define (limit in the measure topology)

$$
M_{4}(E)=\lim _{n \rightarrow \infty} M^{(1 / n)}(E), \quad(E \in \mathcal{E}),
$$

then $M_{4}=\left\{M_{4}(E) \mid E \in \mathcal{E}\right\}$ is a free Lévy basis with free characteristic triplet $(0,0, F)$.

Proof. (i) Let $E$ from $\mathcal{E}$ be given. Since the measure topology is complete, it suffices to show that $\left(M^{(1 / n)}(E)\right)_{n \in \mathbb{N}}$ is a Cauchy sequence in the measure topology, i.e. that $L^{\mathrm{sp}}\left\{M^{(1 / n)}(E)-M^{(1 / m)}(E)\right\} \stackrel{\mathrm{w}}{\rightarrow} \delta_{0}$ as $n, m \rightarrow \infty$. Establishing this condition amounts to verifying that

$$
L^{\mathrm{sp}}\left\{M^{\left(1 / n_{k}\right)}(E)-M^{\left(1 / m_{k}\right)}(E)\right\} \stackrel{\mathrm{w}}{\rightarrow} \delta_{0} \quad \text { as } k \rightarrow \infty
$$

for any sequence $\left(m_{k}, n_{k}\right)_{k \in \mathbb{N}}$ in $\mathbb{N} \times \mathbb{N}$, such that $m_{k} \leq n_{k}$ for all $k$, and such that $m_{k} \rightarrow \infty$ as $k \rightarrow \infty$. Given such a sequence $\left(m_{k}, n_{k}\right)_{k \in \mathbb{N}}$, note first that

$$
\begin{aligned}
M^{\left(1 / n_{k}\right)}(E) & -M^{\left(1 / m_{k}\right)}(E) \\
& =h \cdot P_{F}\left(E \times\left(\left[-\frac{1}{m_{k}},-\frac{1}{n_{k}}\right) \cup\left(\frac{1}{n_{k}}, \frac{1}{m_{k}}\right]\right)\right)-\left[\int_{\left[-\frac{1}{m_{k}},-\frac{1}{n_{k}}\right) \cup\left(\frac{1}{n_{k}}, \frac{1}{m_{k}}\right]} \varsigma(t) F_{E}(\mathrm{~d} t)\right] \mathbf{1}_{\mathcal{M}},
\end{aligned}
$$

and hence by Lemma 6.1 the free characteristic triplet for $M^{\left(1 / n_{k}\right)}(E)-M^{\left(1 / m_{k}\right)}(E)$ is $\left(0,0, \varrho_{k}\right)$, where

$$
\varrho_{k}(B)=F_{E}\left(B \cap\left(\left[-\frac{1}{m_{k}},-\frac{1}{n_{k}}\right) \cup\left(\frac{1}{n_{k}}, \frac{1}{m_{k}}\right]\right)\right)
$$

for any Borel set $B$ in $\mathbb{R}$. It follows from Theorem 2.1 that it suffices to show that

(1) $\int_{\mathbb{R}} f(t) \varrho_{k}(\mathrm{~d} t) \rightarrow 0$ as $k \rightarrow \infty$ for any continuous bounded function $f: \mathbb{R} \rightarrow \mathbb{R}$, which vanishes in a neighborhood of 0 .

(2) $\lim _{\epsilon \downarrow 0}\left(\limsup _{k \rightarrow \infty} \int_{-\epsilon}^{\epsilon} t^{2} \varrho_{k}(\mathrm{~d} t)\right)=0$.

Since $\int_{\mathbb{R}} f(t) \varrho_{k}(\mathrm{~d} t)=\int_{\left[-\frac{1}{m_{k}},-\frac{1}{n_{k}}\right) \cup\left(\frac{1}{n_{k}}, \frac{1}{n_{k}}\right]} f(t) F_{E}(\mathrm{~d} t)$, condition (1) follows from the fact that for all sufficiently large $k$, the set $\left[-\frac{1}{m_{k}},-\frac{1}{n_{k}}\right) \cup\left(\frac{1}{n_{k}}, \frac{1}{m_{k}}\right]$ is contained in the neighborhood of 0 upon which $f$ vanishes. Condition (2), in turn, follows e.g. from the fact that $\int_{-\epsilon}^{\epsilon} t^{2} \varrho_{k}(\mathrm{~d} t) \leq \int_{-\epsilon}^{\epsilon} t^{2} F_{E}(\mathrm{~d} t)$ for any $k$ in $\mathbb{N}$, and here $\int_{-\epsilon}^{\epsilon} t^{2} F_{E}(\mathrm{~d} t) \rightarrow 0$ as $\epsilon \downarrow 0$, since $F_{E}$ is a Lévy measure.

(ii) It follows immediately from the definition of $M_{4}$ that $L^{\mathrm{sp}}\left\{M_{4}(E)\right\} \in \mathcal{J D}(\boxplus)$ for all $E$ in $\mathcal{E}$, since $\mathcal{J D}(\boxplus)$ is closed in the topology for weak convergence and by use of (2.5). For each $n$ in $\mathbb{N}$ the operator $M^{(1 / n)}(E)$ has free characteristic triplet $\left(0,0, F^{(1 / n)}\right)$, where $F^{(1 / n)}$ is given by (6.3). We check next that $M_{4}(E)$ has free characteristic triplet $\left(0,0, F_{E}\right)$ for any $E$ in $\mathcal{E}$. By another application of Theorem 2.1 this is a consequence of the following two facts:

(1) For any continuous bounded function $f: \mathbb{R} \rightarrow \mathbb{R}$ vanishing on a neighborhood of 0 , it holds that

$$
\int_{\mathbb{R}} f \mathrm{~d} F^{(1 / n)}=\int_{\mathbb{R}} f 1_{\left[-\frac{1}{n}, \frac{1}{n}\right]^{c}} \mathrm{~d} F_{E} \underset{n \rightarrow \infty}{\longrightarrow} \int_{\mathbb{R}} f \mathrm{~d} F_{E},
$$

by Dominated Convergence, since $\int_{\mathbb{R}}|f| \mathrm{d} F_{E}<\infty$, because $F_{E}$ is a Lévy measure. 
(2) For any positive number $\epsilon$ we have that

$$
\int_{-\epsilon}^{\epsilon} t^{2} F^{(1 / n)}(\mathrm{d} t)=\int_{-\epsilon}^{\epsilon} t^{2} 1_{\left[-\frac{1}{n}, \frac{1}{n}\right]^{c}}(t) F_{E}(\mathrm{~d} t) \underset{n \rightarrow \infty}{\longrightarrow} \int_{-\epsilon}^{\epsilon} t^{2} F_{E}(\mathrm{~d} t),
$$

by Monotone Convergence. Hence

$$
\lim _{\epsilon \downarrow 0}\left(\limsup _{n \rightarrow \infty} \int_{-\epsilon}^{\epsilon} t^{2} F^{(1 / n)}(\mathrm{d} t)\right)=\lim _{\epsilon \downarrow 0}\left(\int_{-\epsilon}^{\epsilon} t^{2} F_{E}(\mathrm{~d} t)\right)=0,
$$

since $\int_{-1}^{1} t^{2} F_{E}(\mathrm{~d} t)<\infty$.

It remains to show that $M_{4}$ is a free Lévy basis, i.e. to verify the four conditions in Definition 3.1:

(a) We already noted that $L^{\mathrm{sp}}\left\{M_{4}(E)\right\} \in \mathcal{J D}(\boxplus)$ for all $E$ in $\mathcal{E}$.

(b) If $E_{1}, \ldots, E_{n}$ are disjoint sets from $\mathcal{E}$, then $M_{4}\left(E_{1}\right), \ldots, M_{4}\left(E_{n}\right)_{n \in \mathbb{N}}$ are freely independent. This follows from the definition of $M_{4}$ and the corresponding property for $M^{(1 / n)}$, since free independence is preserved under limits in the measure topology (see Proposition 5.4 in [2]).

(c) If $E_{1}, \ldots, E_{n}$ are disjoint sets from $\mathcal{E}$, then $M_{4}\left(\bigcup_{k=1}^{n} E_{k}\right)=\sum_{k=1}^{n} M_{4}\left(E_{k}\right)$. Again this follows from the definition of $M_{4}$ and the corresponding property of $M^{(1 / n)}$, since addition is continuous in the measure topology.

(d) Let $\left(E_{n}\right)_{n \in \mathbb{N}}$ be a decreasing sequence of sets from $\mathcal{E}$, such that $\bigcap_{n \in \mathbb{N}} E_{n}=\emptyset$. Then $L^{\mathrm{sp}}\left\{M_{4}\left(E_{n}\right)\right\} \stackrel{\mathrm{w}}{\rightarrow} \delta_{0}$. Indeed, the free characteristic triplet of $M_{4}\left(E_{n}\right)$ is $\left(0,0, F_{E_{n}}\right)$, and hence by Theorem 2.1 it suffices to check the following two conditions:

(1) $\int_{\mathbb{R}} f \mathrm{~d} F_{E_{n}} \rightarrow 0$ as $n \rightarrow \infty$ for any continuous bounded function $f: \mathbb{R} \rightarrow \mathbb{R}$ vanishing in a neighborhood, say $[-\epsilon, \epsilon]$, of 0 . To see this, note that

$$
\left|\int_{\mathbb{R}} f \mathrm{~d} F_{E_{n}}\right| \leq\|f\|_{\infty} F_{E_{n}}\left([-\epsilon, \epsilon]^{c}\right)=\|f\|_{\infty} F\left(E_{n} \times\left([-\epsilon, \epsilon]^{c}\right)\right) \underset{n \rightarrow \infty}{\longrightarrow} 0,
$$

since $F\left(E_{1} \times\left([-\epsilon, \epsilon]^{c}\right)\right)=F_{E_{1}}\left([-\epsilon, \epsilon]^{c}\right)<\infty$, and $\bigcap_{n \in \mathbb{N}} E_{n} \times\left([-\epsilon, \epsilon]^{c}\right)=\emptyset$.

(2) $\lim _{\epsilon \downarrow 0}\left(\limsup _{n \rightarrow \infty} \int_{-\epsilon}^{\epsilon} t^{2} F_{E_{n}}(\mathrm{~d} t)\right)=0$. To see this, note for any fixed positive $\epsilon$ that

$$
\begin{gathered}
\int_{-\epsilon}^{\epsilon} t^{2} F_{E_{n}}(\mathrm{~d} t)=\int_{E_{n} \times[-\epsilon, \epsilon]} t^{2} F(\mathrm{~d} x, \mathrm{~d} t) \underset{n \rightarrow \infty}{\longrightarrow} 0 \\
\text { because } \int_{E_{1} \times[-\epsilon, \epsilon]} t^{2} F(\mathrm{~d} x, \mathrm{~d} t)=\int_{-\epsilon}^{\epsilon} t^{2} F_{E_{1}}(\mathrm{~d} t)<\infty \text {, and } \bigcap_{n \in \mathbb{N}} E_{n} \times[-\epsilon, \epsilon]=\emptyset
\end{gathered}
$$

This completes the proof.

As an immediate consequence of Lemma 6.3 we obtain the following general version of the Lévy-Itô-decomposition for free Lévy bases. Note that this decomposition coincides with that of Proposition 6.2 under the extra assumption in that proposition.

Corollary 6.4. Consider the framework set up in the beginning of this section, and assume further that there exists a semi-circular free Lévy basis $G_{\Sigma}=\left\{G_{\Sigma}(E) \mid E \in \mathcal{E}\right\}$ in $(\mathcal{M}, \tau)$ with free characteristic triplet $(0, \Sigma, 0)$, which is freely independent of $P_{F}$.

For each $E$ in $\mathcal{E}$ put

$$
\tilde{M}(E)=\Theta(E) \mathbf{1}_{\mathcal{M}}+G_{\Sigma}(E)+M_{4}(E),
$$

where $1_{\mathcal{M}}$ denotes the unit of $\mathcal{M}$ and $M_{4}$ is introduced in Proposition 6.2.

Then $\tilde{M}$ is a free Lévy basis, and for any $E$ in $\mathcal{E}$ the selfadjoint operators $M(E)$ and $\tilde{M}(E)$ share the same spectral distribution. 


\section{Proof of Theorem 3.3}

Throughout this section we consider a nonempty set $X$ and a ring $\mathcal{E}$ of subsets of $X$. We then put

$$
\mathbb{I}=\bigcup_{k \in \mathbb{N}}\left\{\left\{E_{1}, \ldots, E_{k}\right\} \mid E_{1}, \ldots, E_{k} \in \mathcal{E} \backslash\{\emptyset\} \text { and } E_{1}, \ldots, E_{k} \text { are disjoint }\right\} .
$$

We emphasize that we consider an element of II merely as a collection of sets, without paying attention to the order in which these sets appear. Thus we identify an element $\left\{E_{1}, \ldots, E_{k}\right\}$ from II with the element $\left\{E_{\pi(1)}, \ldots, E_{\pi(k)}\right\}$ for any permutation $\pi$ of $\{1, \ldots, k\}$.

We equip II with a partial order " $\leq$ " by declaring that $\left\{E_{1}, \ldots, E_{k}\right\} \leq\left\{F_{1}, \ldots, F_{m}\right\}$ exactly when each $E_{i}$ is a union of some of the $F_{j}$ 's. We note then that " $\leq$ " is an upwardfiltering order, since for $S=\left\{E_{1}, \ldots, E_{k}\right\}$ and $T=\left\{F_{1}, \ldots, F_{m}\right\}$ from $I$ we have that $S, T \leq U$, where $U$ is the element of $\mathbb{I}$ consisting of all non-empty sets in the following family:

$$
E_{i} \cap F_{j}, \quad E_{i} \backslash\left(\bigcup_{j=1}^{m} F_{j}\right), \quad F_{j} \backslash\left(\bigcup_{i=1}^{k} E_{i}\right), \quad(i \in\{1, \ldots, k\}, j \in\{1, \ldots, m\}) .
$$

In the following we consider additionally a family $\{\nu(E, \cdot) \mid E \in \mathcal{E}\}$ of probability measures from $\mathcal{I D}(\boxplus)$, satisfying that

$$
\nu\left(\bigcup_{j=1}^{n} E_{j}, \cdot\right)=\nu\left(E_{1}, \cdot\right) \boxplus \cdots \boxplus \nu\left(E_{n}, \cdot\right),
$$

whenever $E_{1}, \ldots, E_{n}$ are disjoint sets from $\mathcal{E}$. For any set $E$ from $\mathcal{E}$ we denote by $\tau_{E}$ the state on the abelian von Neumann algebra $^{3} L^{\infty}(\nu(E, \cdot))$ given by integration with respect to the probability measure $\nu(E, \cdot)$. Subsequently for any element $S=\left\{E_{1}, \ldots, E_{k}\right\}$ from I, we let $\left(\mathcal{M}_{S}, \tau_{S}\right)$ denote the $W^{*}$-reduced free product of the $W^{*}$-probability spaces $\left(L^{\infty}\left(\nu\left(E_{j}, \cdot\right)\right), \tau_{E_{j}}\right), j=1, \ldots, k$ (see [21] for details).

Lemma 7.1. For any element $S=\left\{E_{1}, \ldots, E_{k}\right\}$ of $\mathbb{I}$ there exist freely independent operators $M_{S}\left(E_{1}\right), \ldots, M_{S}\left(E_{k}\right)$ from $\overline{\mathcal{M}}_{S}$, which generate $\mathcal{M}_{S}$ as a von Neumann algebra ${ }^{4}$, and such that

$$
L^{\mathrm{sp}}\left\{M\left(E_{j}\right)\right\}=\nu\left(E_{j}, \cdot\right), \quad(j=1, \ldots, k) .
$$

Proof. For each $j$ in $\{1, \ldots, k\}$ we have a canonical embedding $\iota_{j}: L^{\infty}\left(\nu\left(E_{j}, \cdot\right)\right) \hookrightarrow \mathcal{M}_{S}$, such that $\tau_{E_{j}}=\tau_{S} \circ \iota_{j}$ (see [21]). By Proposition A.4 $\iota_{j}$ gives rise to a $*$-homomorphism $\bar{\iota}_{j}: \overline{L^{\infty}\left(\nu\left(E_{j}, \cdot\right)\right)} \rightarrow \overline{\mathcal{M}}_{S}$. We then define

$$
M_{S}\left(E_{j}\right)=\bar{\iota}_{j}\left(\operatorname{id}_{\mathbb{R}}\right), \quad(j=1, \ldots, k),
$$

where $i d_{\mathbb{R}}$ denotes the identity function on $\mathbb{R}$ considered as an element of $\overline{L^{\infty}\left(\nu\left(E_{j}, \cdot\right)\right)}$. By Proposition A.4 the range of $\bar{\iota}_{j}$ equals the class of operators affiliated with the von Neumann algebra $\iota_{j}\left(L^{\infty}\left(\nu\left(E_{j}, \cdot\right)\right)\right.$, so in particular $M_{S}\left(E_{j}\right)$ is affiliated with that von Neumann algebra. By construction of $\left(\mathcal{M}_{S}, \tau_{S}\right)$ the algebras $\iota_{j}\left(L^{\infty}\left(\nu\left(E_{j}, \cdot\right)\right), j=1, \ldots, n\right.$, are free in $\left(\mathcal{M}_{S}, \tau_{S}\right)$, so in particular $M_{S}\left(E_{1}\right), \ldots, M_{S}\left(E_{k}\right)$ are freely independent with respect to $\tau$. For any $f$ in $\mathcal{B F}_{b}(\mathbb{R})$ we note next (cf. Proposition A.4) that

$$
f\left(M_{S}\left(E_{j}\right)\right)=f\left(\bar{\iota}_{j}\left(\operatorname{id}_{\mathbb{R}}\right)\right)=\bar{\iota}_{j}\left(f\left(\operatorname{id}_{\mathbb{R}}\right)\right)=\bar{\iota}_{j}(f)=\iota_{j}(f),
$$

\footnotetext{
${ }^{3} L^{\infty}(\nu(E, \cdot))$ is the vector space of all $\nu(E, \cdot)$-essentially bounded functions $f: X \rightarrow \mathbb{C}$ identified up to $\nu(E, \cdot)$-null sets.

${ }^{4}$ Operators $T_{1}, \ldots, T_{k}$ affiliated with a von Neumann algebra $\mathcal{M}$ are said to generate $\mathcal{M}$ as a von Neumann algebra, if $\mathcal{M}$ is the smallest von Neumann algebra on the considered Hilbert space containing the family $\bigcup_{j=1}^{k}\left\{f\left(T_{j}\right) \mid f \in \mathcal{B F}_{b}(\mathbb{R})\right\}$.
} 
and hence $M_{S}\left(E_{j}\right)$ generates $\iota_{j}\left(L^{\infty}\left(\nu\left(E_{j}, \cdot\right)\right)\right.$ as a von Neumann algebra. This further implies that $\left\{M_{S}\left(E_{1}\right), \ldots, M_{S}\left(E_{n}\right)\right\}$ generates $\mathcal{M}_{S}$ as a von Neumann algebra (cf. [21, Definition 1.6.1]). For any $j$ in $\{1, \ldots, k\}$ and any function $f$ from $\mathcal{B F}_{b}(\mathbb{R})$ we note finally that

$$
\tau_{S}\left[f\left(M_{S}\left(E_{j}\right)\right)\right]=\tau_{S}\left[\iota_{j}(f)\right]=\tau_{E_{j}}(f)=\int_{\mathbb{R}} f(t) \nu\left(E_{j}, \mathrm{~d} t\right),
$$

verifying that $L^{\mathrm{sp}}\left\{M_{S}\left(E_{j}\right)\right\}=\nu\left(E_{j}, \cdot\right)$.

Lemma 7.2. Assume that $S=\left\{E_{1}, \ldots, E_{k}\right\}$ and $T=\left\{F_{1}, \ldots, F_{m}\right\}$ are elements of II such that $S \leq T$. Then there exists a normal $*$-homomorphism $\iota_{S, T}: \mathcal{M}_{S} \rightarrow \mathcal{M}_{T}$ such that $\tau_{S}=\tau_{T} \circ \iota_{S, T}$.

Specifically it holds for any $i$ in $\{1, \ldots, k\}$ (with notation from Lemma 7.1) that

$$
\bar{\iota}_{S, T}\left(M_{S}\left(E_{i}\right)\right)=M_{T}\left(F_{j(i, 1)}\right)+\cdots+M_{T}\left(F_{j\left(i, l_{i}\right)}\right),
$$

whenever $E_{i}=F_{j(i, 1)} \cup \cdots \cup F_{j\left(i, l_{i}\right)}$ for suitable $j(i, 1), \ldots, j\left(i, l_{i}\right)$ from $\{1, \ldots, m\}$.

Proof. We adopt the notation from Lemma 7.1. Given any $i$ in $\{1,2, \ldots, k\}$ we may, since $S \leq T$, write $E_{i}$ (unambiguously) as $F_{j(i, 1)} \cup \cdots \cup F_{j\left(i, l_{i}\right)}$ for suitable $j(i, 1), \ldots, j\left(i, l_{i}\right)$ from $\{1, \ldots, m\}$. Since the operators $M_{T}\left(F_{j(i, 1)}\right), \ldots, M_{T}\left(F_{j\left(i, l_{i}\right)}\right)$ are freely independent, it follows then that

$$
\begin{aligned}
L^{\mathrm{sp}}\left\{M_{T}\left(F_{j(i, 1)}\right)+\cdots+M_{T}\left(F_{j\left(i, l_{i}\right)}\right)\right\} & =\nu\left(F_{j(i, 1)}, \cdot\right) \boxplus \cdots \boxplus \nu\left(F_{j\left(i, l_{i}\right)}, \cdot\right) \\
& =\nu\left(F_{j(i, 1)} \cup \cdots \cup F_{j\left(i, l_{i}\right)}, \cdot\right)=\nu\left(E_{i}, \cdot\right)=L^{\mathrm{sp}}\left\{M_{S}\left(E_{i}\right)\right\} .
\end{aligned}
$$

Note also that since all the operators $M_{T}\left(F_{1}\right), \ldots, M_{T}\left(F_{m}\right)$ are freely independent, the sums $M_{T}\left(F_{j(i, 1)}\right)+\cdots+M_{T}\left(F_{j\left(i, l_{i}\right)}\right), i=1, \ldots, k$, are also freely independent. Indeed, for each $i$ the sum $M_{T}\left(F_{j(i, 1)}\right)+\cdots+M_{T}\left(F_{j\left(i, l_{i}\right)}\right)$ is affiliated with the von Neumann algebra generated by $L^{\infty}\left(\nu\left(F_{j(i, 1)}, \cdot\right), \ldots, L^{\infty}\left(\nu\left(F_{j\left(i, l_{i}\right)}, \cdot\right)\right.\right.$ considered as sub-algebras of $\mathcal{M}_{T}$. And by [21, Proposition 2.5.5] these von Neumann subalgebras are free in $\left(\mathcal{M}_{T}, \tau_{T}\right)$ for varying $i$. It follows that the two families of operators:

$$
\bigcup_{i=1}^{k}\left\{f\left(M_{T}\left(F_{j(i, 1)}\right)+\cdots+M_{T}\left(F_{j\left(i, l_{i}\right)}\right)\right) \mid f \in \mathcal{B} \mathcal{F}_{b}(\mathbb{R})\right\}
$$

and

$$
\left.\bigcup_{i=1}^{k}\left\{f\left(M_{S}\left(E_{i}\right)\right)\right) \mid f \in \mathcal{B F}_{b}(\mathbb{R})\right\}
$$

have the same $*$-distribution, and since $M_{S}\left(E_{1}\right), \ldots, M_{S}\left(E_{k}\right)$ generate $\mathcal{M}_{S}$ as a von Neumann algebra, it follows thus from Proposition A.1 that there exists a normal, injective $*$-homomorphism $\iota_{S, T}: \mathcal{M}_{S} \rightarrow \mathcal{M}_{T}$ such that

$$
\bar{\iota}_{S, T}\left(f\left(M_{S}\left(E_{i}\right)\right)\right)=f\left(M_{T}\left(F_{j(i, 1)}\right)+\cdots+M_{T}\left(F_{j\left(i, l_{i}\right)}\right)\right)
$$

for any $i$ in $\{1, \ldots, k\}$ and $f$ in $\mathcal{B F}_{b}(\mathbb{R})$. In addition $\tau_{S}=\tau_{T} \circ \iota_{S, T}$.

To establish (7.2) we consider for each $n$ in $\mathbb{N}$ the function $f_{n}: \mathbb{R} \rightarrow \mathbb{R}$ defined by:

$$
f_{n}(t)=t 1_{[-n, n]}(t)-n 1_{(-\infty,-n)}(t)+n 1_{(n, \infty)}(t), \quad(t \in \mathbb{R}) .
$$

Then $f_{n}(t) \rightarrow t$ as $n \rightarrow \infty$ for all $t$ in $\mathbb{R}$, and this implies that $f_{n}\left(M_{S}\left(E_{i}\right)\right) \stackrel{\mathrm{P}}{\rightarrow} M_{S}\left(E_{i}\right)$ and that $f_{n}\left(M_{T}\left(F_{j(i, 1)}\right)+\cdots+M_{T}\left(F_{j\left(i, i_{l}\right)}\right)\right) \stackrel{\mathrm{P}}{\rightarrow} M_{T}\left(F_{j(i, 1)}\right)+\cdots+M_{T}\left(F_{j\left(i, i_{l}\right)}\right)$ as $n \rightarrow \infty$ (cf. 
the calculation (A.4) in the proof of Proposition A.4). From formula (A.3) in that same proof it follows then further that

$$
\begin{aligned}
\bar{\iota}_{S, T}\left(M_{S}\left(E_{i}\right)\right) & =\mathrm{P}-\lim _{n \rightarrow \infty} \iota_{S, T}\left(f_{n}\left(M_{S}\left(E_{i}\right)\right)\right)=\mathrm{P}-\lim _{n \rightarrow \infty} f_{n}\left(M_{T}\left(F_{j(i, 1)}\right)+\cdots+M_{T}\left(F_{j\left(i, i_{l}\right)}\right)\right) \\
& =M_{T}\left(F_{j(i, 1)}\right)+\cdots+M_{T}\left(F_{j\left(i, i_{l}\right)}\right)
\end{aligned}
$$

as desired. This completes the proof.

Lemma 7.3. The family $\left(\mathcal{M}_{S}, \tau_{S}\right)_{S \in \mathbb{I}}$ of $W^{*}$-probability spaces equipped with the family $\left\{\iota_{S, T} \mid S, T \in \mathbb{I}, S \leq T\right\}$ of $*$-homomorphisms described in Lemma 7.2 forms a directed system of $W^{*}$-algebras and injective, normal $*$-homomorphisms.

Proof. Given $R, S, T$ in II such that $R \leq S \leq T$, we must show that $\iota_{R, T}=\iota_{S, T} \circ \iota_{R, S}$. Writing $R=\left\{D_{1}, \ldots, D_{m}\right\}, S=\left\{E_{1}, \ldots, E_{k}\right\}$ and $T=\left\{F_{1}, \ldots, F_{l}\right\}$ for suitable $D_{h}, E_{i}, F_{j}$ from $\mathcal{E} \backslash\{\emptyset\}$, we know that

$$
\begin{aligned}
D_{h} & =E_{i(h, 1)} \cup \cdots \cup E_{i\left(h, k_{h}\right)}, \quad(h=1, \ldots, m), \\
E_{i} & =F_{j(i, 1)} \cup \cdots \cup F_{j\left(i, l_{i}\right)}, \quad(i=1, \ldots, k),
\end{aligned}
$$

for suitable $i(h, 1), \ldots, i\left(h, k_{h}\right)$ in $\{1, \ldots, k\}$ and $j(i, 1), \ldots, j\left(i, l_{i}\right)$ from $\{1, \ldots, l\}$. Then

$$
\begin{aligned}
\bar{\iota}_{R, T}\left(M_{R}\left(D_{h}\right)\right) & =\sum_{r=1}^{l_{i(h, 1)}} M_{T}\left(F_{j(i(h, 1), r)}\right)+\cdots+\sum_{r=1}^{l_{i\left(h, k_{h}\right)}} M_{T}\left(F_{j\left(i\left(h, k_{h}\right), r\right)}\right) \\
& =\bar{\iota}_{S, T}\left(M_{S}\left(E_{i(h, 1)}\right)\right)+\cdots+\bar{\iota}_{S, T}\left(M_{S}\left(E_{i\left(h, k_{h}\right)}\right)\right) \\
& =\bar{\iota}_{S, T}\left(\left(M_{S}\left(E_{i(h, 1)}\right)+\cdots+M_{S}\left(E_{i\left(h, k_{h}\right)}\right)\right)\right. \\
& =\bar{\iota}_{S, T}\left(\bar{\iota}_{R, S}\left(D_{h}\right)\right) .
\end{aligned}
$$

For any $f$ in $\mathcal{B F}_{b}(\mathbb{R})$ it follows then (cf. Proposition A.4) that

$$
\begin{aligned}
\iota_{R, T}\left(f\left(M_{R}\left(D_{h}\right)\right)\right) & =f\left(\bar{\iota}_{R, T}\left(M_{R}\left(D_{h}\right)\right)\right)=f\left(\bar{\iota}_{S, T} \circ \bar{\iota}_{R, S}\left(M_{R}\left(D_{h}\right)\right)\right) \\
& =\iota_{S, T}\left(f\left(\bar{\iota}_{R, S}\left(M_{R}\left(D_{h}\right)\right)\right)\right)=\iota_{S, T} \circ \iota_{R, S}\left(f\left(M_{R}\left(D_{h}\right)\right)\right) .
\end{aligned}
$$

Since $\mathcal{M}_{R}$ is generated as a von Neumann algebra by the family

$$
\bigcup_{h=1}^{m}\left\{f\left(M_{R}\left(D_{h}\right)\right) \mid f \in \mathcal{B F}_{b}(\mathbb{R})\right\},
$$

and since $\iota_{R, T}$ and $\iota_{S, T} \circ \iota_{R, S}$ are both normal, it follows by an application of Kaplansky's Density Theorem that $\iota_{R, T}=\iota_{R, S} \circ \iota_{S, T}$, as desired.

Proof of Theorem 3.3. We note first that assertions (ii) and (iii) are direct consequences of (i). To prove (i), we consider the directed system (cf. Lemma 7.3)

$$
\left(\mathcal{M}_{S}, \tau_{S}\right)_{S \in \mathbb{I}}, \quad\left\{\iota_{S, T} \mid S, T \in \mathbb{I}, S \leq T\right\}
$$

of $W^{*}$-probability spaces and trace preserving $*$-homomorphisms. Using Proposition A.3, there exists a $W^{*}$-probability space $(\mathcal{M}, \tau)$ and injective, normal $*$-homomorphisms $\iota_{S}: \mathcal{M}_{S} \rightarrow \mathcal{M}(S \in \mathbb{I})$, satisfying that $\tau_{S}=\tau \circ \iota_{S}$ for all $S$ in $\mathbb{I}$, and that $\iota_{S}=\iota_{T} \circ \iota_{S, T}$ for any $S, T$ in II such that $S \leq T$. We now define

$$
M(\emptyset)=0, \quad \text { and } \quad M(E)=\bar{\iota}_{\{E\}}\left(M_{\{E\}}(E)\right) \text { for } E \text { in } \mathcal{E} \backslash\{\emptyset\},
$$


where $M_{\{E\}}(E)$ denotes the identity function $\operatorname{id}_{\mathbb{R}}$ on $\mathbb{R}$ considered as an element of $\overline{L^{\infty}(\nu(E, \cdot))}=\overline{\mathcal{M}}_{\{E\}}$. We will show that the family $\{M(E) \mid E \in \mathcal{E}\}$ satisfies the conditions (a)-(b) in Definition 3.1 and that $L^{\mathrm{sp}}\{M(E)\}=\nu(E, \cdot)$ for all $E$ in $\mathcal{E}$.

(a) Assume that $E_{1}, \ldots, E_{r}$ are disjoint sets from $\mathcal{E} \backslash\{\emptyset\}$, and put $S=\left\{E_{1}, \ldots, E_{r}\right\} \in \mathbb{I}$. Consider further arbitrary functions $f_{1}, \ldots, f_{r}$ from $\mathcal{B F}_{b}(\mathbb{R})$. We must show that the bounded operators $f_{1}\left(M\left(E_{1}\right)\right), \ldots, f_{r}\left(M\left(E_{r}\right)\right)$ are freely independent with respect to $\tau$. For any polynomial $p$ in $r$ non-commuting variables we note (cf. Proposition A.4) that

$$
\begin{aligned}
\tau\left[p \left(f_{1}\left(M\left(E_{1}\right)\right)\right.\right. & \left.\left.\ldots, f_{r}\left(M\left(E_{r}\right)\right)\right)\right] \\
& =\tau\left[p\left(f_{1}\left(\bar{\iota}_{\left\{E_{1}\right\}}\left(M_{\left\{E_{1}\right\}}\left(E_{1}\right)\right)\right), \ldots, f_{r}\left(\bar{\iota}_{\left\{E_{r}\right\}}\left(M_{\left\{E_{r}\right\}}\left(E_{r}\right)\right)\right)\right)\right] \\
& =\tau\left[p\left(\iota_{\left\{E_{1}\right\}}\left(f_{1}\left(M_{\left\{E_{1}\right\}}\left(E_{1}\right)\right)\right), \ldots, \iota_{\left\{E_{r}\right\}}\left(f_{r}\left(M_{\left\{E_{r}\right\}}\left(E_{r}\right)\right)\right)\right)\right] \\
& =\tau\left[p\left(\iota_{S} \circ \iota_{\left\{E_{1}\right\}, S}\left(f_{1}\right), \ldots, \iota_{S} \circ \iota_{\left\{E_{r}\right\}, S}\left(f_{r}\right)\right)\right] \\
& =\tau\left[\iota_{S}\left(p\left(\iota_{\left\{E_{1}\right\}, S}\left(f_{1}\right), \ldots, \iota_{\left\{E_{r}\right\}, S}\left(f_{r}\right)\right)\right)\right] \\
& =\tau_{S}\left[p\left(\iota_{\left\{E_{1}\right\}, S}\left(f_{1}\right), \ldots, \iota_{\left\{E_{r}\right\}, S}\left(f_{r}\right)\right)\right] .
\end{aligned}
$$

For each $j$ in $\{1, \ldots, r\}$ recall that $\iota_{\left\{E_{j}\right\}, S}$ is the canonical embedding of $\mathcal{M}_{\left\{E_{j}\right\}}=$ $L^{\infty}\left(\nu\left(E_{j}, \cdot\right)\right)$ into the reduced free product $\mathcal{M}_{S}=L^{\infty}\left(\nu\left(E_{1}, \cdot\right)\right) * \cdots * L^{\infty}\left(\nu\left(E_{r}, \cdot\right)\right)$. Hence the ranges of $\iota_{\left\{E_{1}\right\}, S}, \cdots, \iota_{\left\{E_{r}\right\}, S}$ are free in $\left(\mathcal{M}_{S}, \tau_{S}\right)$, and in particular $\iota_{\left\{E_{1}\right\}, S}\left(f_{1}\right), \ldots$, $\iota_{\left\{E_{r}\right\}, S}\left(f_{r}\right)$ are freely independent with respect to $\tau_{S}$. Since (7.5) holds for any polynomial $p$ in $r$ non-commuting variables, it follows then that $f_{1}\left(M\left(E_{1}\right)\right), \ldots, f_{r}\left(M\left(E_{r}\right)\right)$ are freely independent with respect to $\tau$.

(b) Let $E_{1}, \ldots, E_{r}$ be disjoint sets from $\mathcal{E} \backslash\{\emptyset\}$, and put $E=\bigcup_{j=1}^{r} E_{j}$ and $S=\left\{E_{1}, \ldots\right.$, $\left.E_{r}\right\} \in \mathbb{I}$. We must show that $M(E)=M\left(E_{1}\right)+\cdots+M\left(E_{r}\right)$. Using Corollary A.5 we find that

$$
\begin{aligned}
M\left(E_{1}\right)+ & \cdots+M\left(E_{r}\right) \\
& =\bar{\iota}_{\left\{E_{1}\right\}}\left(\operatorname{id}_{\mathbb{R}}\right)+\cdots+\bar{\iota}_{\left\{E_{r}\right\}}\left(\operatorname{id}_{\mathbb{R}}\right)=\overline{\iota_{S} \circ \iota_{\left\{E_{1}\right\}, S}}\left(\operatorname{id}_{\mathbb{R}}\right)+\cdots+\overline{\iota_{S} \circ \iota_{\left\{E_{r}\right\}, S}}\left(\operatorname{id}_{\mathbb{R}}\right) \\
& =\bar{\iota}_{S} \circ \bar{\iota}_{\left\{E_{1}\right\}, S}\left(\operatorname{id}_{\mathbb{R}}\right)+\cdots+\bar{\iota}_{S} \circ \bar{\iota}_{\left\{E_{r}\right\}, S}\left(\operatorname{id}_{\mathbb{R}}\right)=\bar{\iota}_{S}\left(M_{S}\left(E_{1}\right)+\cdots+M_{S}\left(E_{r}\right)\right) \\
& =\bar{\iota}_{S}\left(\bar{\iota}_{\{E\}, S}\left(M_{\{E\}}(E)\right)=\overline{\iota_{S} \circ \iota_{\{E\}, S}}\left(\operatorname{id}_{\mathbb{R}}\right)\right. \\
& =\bar{\iota}_{\{E\}}\left(\operatorname{id}_{\mathbb{R}}\right)=M(E),
\end{aligned}
$$

where in the first, fourth, fifth and last equality we applied (7.4), (7.2), (7.2) and (7.4), respectively.

To show finally that $L^{\mathrm{sp}}\{M(E)\}=\nu(E, \cdot)$ for all $E$ in $\mathcal{E}$, we assume without loss of generality that $E \neq \emptyset$. Then since $\tau_{\{E\}}=\tau \circ \iota_{\{E\}}$ we find for any function $f$ in $\mathcal{B F}_{b}(\mathbb{R})$ that

$$
\tau[f(M(E))]=\tau\left[f\left(\bar{\iota}_{\{E\}}\left(M_{\{E\}}(E)\right)\right)\right]=\tau\left[\iota_{\{E\}}\left(f\left(M_{\{E\}}(E)\right)\right)\right]=\tau_{\{E\}}(f)=\int_{\mathbb{R}} f \mathrm{~d} \nu(E, \cdot),
$$

which proves the desired identity. This completes the proof.

\section{A Appendix: Von Neumann algebra preliminaries}

To accommodate potential readers with limited background in the theory of operator algebras, we start by recalling briefly various basic concepts from that theory. For a 
Completely random measures and Lévy bases in free probability

thorough introduction to operator algebras we refer to the classical texts [9, 10]. First of all an algebra (over $\mathbb{C}$ ) is a vector space $\mathcal{A}$ over $\mathbb{C}$, which is also furnished with an associative multiplication satisfying the usual distributive laws in relation to the linear operations. One may think of the matrix algebra $M_{n}(\mathbb{C})$ as a concrete example. As in this particular case the multiplication is generally not assumed to be commutative. We say that $\mathcal{A}$ is a $*$-algebra, if it is additionally equipped with an involution (or $*$-operation) $a \mapsto a^{*}: \mathcal{A} \rightarrow \mathcal{A}$, satisfying that $(a+b)^{*}=a^{*}+b^{*},(z a)^{*}=\bar{z} a^{*},(a b)^{*}=b^{*} a^{*}$ and $\left(a^{*}\right)^{*}=a$ for all $a, b$ in $\mathcal{A}$ and $z$ in $\mathbb{C}$.

A $C^{*}$-algebra is a $*$-algebra $\mathcal{A}$, which is also a Banach space with respect to a norm $\|\cdot\|$, satisfying additionally that $\|a b\| \leq\|a\|\|b\|$ and $\left\|a^{*} a\right\|=\|a\|^{2}$ for all $a, b$ in $\mathcal{A}$. Again, $M_{n}(\mathbb{C})$ provides a (finite dimensional) example of a $C^{*}$-algebra, and more generally the space $\mathcal{B}(\mathcal{H})$ of continuous linear mappings $T: \mathcal{H} \rightarrow \mathcal{H}$ on a Hilbert space $\mathcal{H}$ is a canonical example of a $C^{*}$-algebra. In fact any $C^{*}$-algebra may be identified with a norm closed, *-invariant subalgebra of $\mathcal{B}(\mathcal{H})$. As in $[9,10]$ we shall generally assume that a $C^{*}$-algebra $\mathcal{A}$ comes equipped with a multiplicative neutral element $\mathbf{1}_{\mathcal{A}}$. If $\mathcal{A}$ and $\mathcal{B}$ are two $C^{*}$-algebras, a linear mapping $\varphi: \mathcal{A} \rightarrow \mathcal{B}$ is called a $*$-homomorphism, if $\varphi\left(\mathbf{1}_{\mathcal{A}}\right)=\mathbf{1}_{\mathcal{B}}$, $\varphi(a b)=\varphi(a) \varphi(b)$, and $\varphi\left(a^{*}\right)=\varphi(a)^{*}$ for all $a, b$ in $\mathcal{A}$.

A von Neumann algebra acting on a Hilbert space $\mathcal{H}$ is a $*$-invariant subalgebra $\mathcal{M}$ of $\mathcal{B}(\mathcal{H})$, which is closed in the weak operator topology, i.e. the weak topology on $\mathcal{B}(\mathcal{H})$ induced by the family $\left\{\omega_{\xi, \eta} \mid \xi, \eta \in \mathcal{H}\right\}$ of linear functionals given by

$$
\omega_{\xi, \eta}(a)=\langle a \xi, \eta\rangle, \quad(a \in \mathcal{B}(\mathcal{H})) .
$$

As this topology is weaker than that induced by the $C^{*}$-norm on $\mathcal{B}(\mathcal{H})$, a von Neumann algebra is automatically a $C^{*}$-algebra. If $\mathcal{M}$ and $\mathcal{N}$ are two von Neumann-algebras (possibly acting on different Hilbert spaces) and $\varphi: \mathcal{N} \rightarrow \mathcal{N}$ is a $*$-homomorphism, then $\varphi$ is said to be normal if its restriction to the unit ball of $\mathcal{N}$ is continuous with respect to the weak operator topologies on $\mathcal{M}$ and $\mathcal{N}$.

With the above basic concepts in place, we recall next that a $W^{*}$-probability space is a pair $(\mathcal{M}, \tau)$, where $\mathcal{M}$ is a von Neumann algebra (acting on some Hilbert space), and $\tau$ is a faithful, normal, tracial state on $\mathcal{M}$. Specifically $\tau$ is a linear mapping from $\mathcal{M}$ into $\mathbb{C}$, which is continuous on the unit ball of $\mathcal{M}$ with respect to the weak operator topology and satisfies the following conditions: $\tau\left(a^{*} a\right)>0$ for all $a$ in $\mathcal{M} \backslash\{0\}, \tau(a b)=\tau(b a)$ for all $a, b$ in $\mathcal{M}$, and $\tau\left(\mathbf{1}_{\mathcal{M}}\right)=1$.

If $I$ is an arbitrary non-empty index set, and $\left(x_{i}\right)_{i \in I}$ is a corresponding family of operators in a $W^{*}$-probability space $(\mathcal{M}, \tau)$, then the $*$-distribution of $\left(x_{i}\right)_{i \in I}$ is the collection of all complex numbers in the form

$$
\tau\left(z_{1}^{p_{1}} z_{2}^{p_{2}} \cdots z_{n}^{p_{n}}\right)
$$

where $n \in \mathbb{N}, p_{1}, \ldots, p_{n} \in \mathbb{N}$ and $z_{1}, \ldots, z_{n} \in\left\{x_{i} \mid i \in I\right\} \cup\left\{x_{i}^{*} \mid i \in I\right\}$.

Proposition A.1. Let $(\mathcal{M}, \tau)$ and $(\mathcal{N}, \psi)$ be $W^{*}$-probability spaces, let $I$ be a non-empty index set, and assume that $\left(x_{i}\right)_{i \in I}$ and $\left(y_{i}\right)_{i \in I}$ are families of operators from $\mathcal{M}$ and $\mathcal{N}$, respectively. Let $\mathcal{M}_{0}$ denote the von Neumann subalgebra of $\mathcal{M}$ generated by $\left(x_{i}\right)_{i \in I}$, and let $\mathcal{N}_{0}$ denote the von Neumann subalgebra of $\mathcal{N}$ generated by $\left(y_{i}\right)_{i \in I}$.

If the $*$-distribution of $\left(x_{i}\right)_{i \in I}$ (with respect to $\tau$ ) equals that of $\left(y_{i}\right)_{i \in I}$ (with respect to $\psi$ ), then there exists a normal $*$-isomorphism $\Phi$ of $\mathcal{M}_{0}$ onto $\mathcal{N}_{0}$, such that $\tau=\psi \circ \Phi$ on $\mathcal{M}_{0}$, and such that $\Phi\left(x_{i}\right)=y_{i}$ for all $i$ in $I$.

For the proof of Proposition A.1 we refer to [12, Theorem 2 in Section 6.5] or [20, Remark 1.8].

Corollary A.2. Let $(\mathcal{M}, \tau)$ and $(\mathcal{N}, \psi)$ be $W^{*}$-probability spaces, and let $\Phi: \mathcal{M} \rightarrow \mathcal{N}$ be a $*$-homomorphism such that $\tau=\psi \circ \Phi$. Then $\Phi$ is automatically normal and injective, and $\Phi(\mathcal{M})$ is a von Neumann subalgebra of $\mathcal{N}$. 
Proof. Since $\tau=\psi \circ \Phi$, the two families of operators $\mathcal{M}$ and $\Phi(\mathcal{M})$ (indexed by $\mathcal{M}$ ) have the same *-distribution. By application of Proposition A.1, we obtain thus a normal *-isomorphism $\tilde{\Phi}$ of the von Neumann subalgebra generated by $\mathcal{M}$ (i.e. $\mathcal{M}$ itself) onto the von Neumann subalgebra of $\mathcal{N}$ generated by $\Phi(\mathcal{M})$, such that $\tilde{\Phi}(a)=\Phi(a)$ for all $a$ in $\mathcal{M}$. Obviously then $\Phi=\tilde{\Phi}$, so $\Phi$ is normal and injective. In addition $\Phi(\mathcal{M})=\tilde{\Phi}(\mathcal{M})$, which is a von Neumann algebra.

Recall that a partial order " $\leq$ " on a set $\delta$ is called upward filtering, if, for any elements $S, T$ in $\mathcal{S}$, there exists an element $U$ in $\mathcal{S}$ such that $S \leq U$ and $T \leq U$.

The following result essentially amounts to the existence of inductive limits in the category of $W^{*}$-probability spaces.

Proposition A.3. Consider a set $\mathcal{S}$ equipped with an upward filtering partial order " $\leq$ ". Consider additionally a corresponding family $\left(\mathcal{M}_{S}, \tau_{S}\right)_{S \in \mathcal{S}}$ of $W^{*}$-probability spaces, and assume that whenever $S, T \in \mathcal{S}$, such that $S \leq T$, there is a *-homomorphism $\Phi_{S, T}: \mathcal{M}_{S} \rightarrow \mathcal{M}_{T}$ such that $\tau_{S}=\tau_{T} \circ \Phi_{S, T}$.

Then there exists a $W^{*}$-probability space $(\mathcal{M}, \tau)$ and injective normal $*$-homomorphisms $\Phi_{S}: \mathcal{M}_{S} \rightarrow \mathcal{M}(S \in \mathcal{S})$, such that

$$
\tau \circ \Phi_{S}=\tau_{S} \text { for all } S \text { in } \mathcal{S},
$$

and

$$
\Phi_{T} \circ \Phi_{S, T}=\Phi_{S} \quad \text { for all } S, T \text { in } \mathcal{S} \text {, such that } S \leq T .
$$

In addition $\mathcal{M}$ is generated as a von Neumann algebra by the *-subalgebra $\bigcup_{S \in \mathcal{S}} \Phi_{S}\left(\mathcal{M}_{S}\right)$.

The properties listed above characterize $(\mathcal{M}, \tau)$ up to trace preserving $*$-isomorphisms.

Proof. From Corollary A.2 we know that $\Phi_{S, T}$ is normal and injective for any $S, T$ in $\mathcal{S}$ such that $S \leq T$, and moreover $\Phi_{S, T}\left(\mathcal{M}_{S}\right)$ is a von Neumann subalgebra of $\mathcal{M}_{T}$. Now let $\mathcal{M}^{0}$ be the $C^{*}$-algebra inductive limit of the directed system

$$
\left\{\mathcal{M}_{S} \mid S \in \mathcal{S}\right\}, \quad\left\{\Phi_{S, T} \mid S, T \in \mathcal{S}, S \leq T\right\}
$$

(see [10, Proposition 11.4.1] for details). Then for any $S$ in $\mathcal{S}$ there is a $*$-monomorphism $\Phi_{S}^{0}: \mathcal{M}_{S} \rightarrow \mathcal{M}^{0}$, such that $\Phi_{T}^{0} \circ \Phi_{S, T}=\Phi_{S}^{0}$, whenever $S, T \in \mathcal{S}$ such that $S \leq T$. Putting $\mathcal{M}^{00}=\bigcup_{S \in \mathcal{S}} \Phi_{S}^{0}\left(\mathcal{M}_{S}\right)$, we may then define a linear functional $\tau^{00}: \mathcal{M}^{00} \rightarrow \mathbb{C}$ such that

$$
\tau_{S}=\tau^{00} \circ \Phi_{S}^{0} \quad \text { for all } S \text { in } \mathcal{S} .
$$

Indeed, if $a \in \Phi_{S}^{0}\left(\mathcal{M}_{S}\right) \cap \Phi_{T}^{0}\left(\mathcal{M}_{T}\right)$ for $S, T$ in $\mathcal{S}$, we have that $a=\Phi_{S}^{0}\left(a^{\prime}\right)=\Phi_{T}^{0}\left(a^{\prime \prime}\right)$ for suitable $a^{\prime}$ in $S$ and $a^{\prime \prime}$ in $T$, and we must show that $\tau_{S}\left(a^{\prime}\right)=\tau_{T}\left(a^{\prime \prime}\right)$. Since " $\leq$ " is upward filtering, we may choose an element $U$ of $\mathcal{S}$, such that $S, T \leq U$. Now

$$
\Phi_{U}^{0} \circ \Phi_{S, U}\left(a^{\prime}\right)=\Phi_{S}^{0}\left(a^{\prime}\right)=\Phi_{T}^{0}\left(a^{\prime \prime}\right)=\Phi_{U}^{0} \circ \Phi_{T, U}\left(a^{\prime \prime}\right),
$$

so the injectivity of $\Phi_{U}^{0}$ implies that $\Phi_{S, U}\left(a^{\prime}\right)=\Phi_{T, U}\left(a^{\prime \prime}\right)$, and therefore

$$
\tau_{S}\left(a^{\prime}\right)=\tau_{U} \circ \Phi_{S, U}\left(a^{\prime}\right)=\tau_{U} \circ \Phi_{T, U}\left(a^{\prime \prime}\right)=\tau_{T}\left(a^{\prime \prime}\right),
$$

as desired. Thus (A.1) gives rise to a well-defined mapping $\tau^{00}: \mathcal{M}^{00} \rightarrow \mathbb{C}$, and by similar reasoning it follows that $\tau^{00}$ is a linear, positive, tracial and norm-decreasing functional on $\mathcal{M}^{00}$. Since $\mathcal{M}^{00}$ is dense in $\mathcal{M}^{0}$ with respect to the operator norm (cf. [10, Proposition 11.4.1]), $\tau^{00}$ thus extends to a linear, tracial, norm-decreasing functional $\tau^{0}: \mathcal{M}^{0} \rightarrow \mathbb{C}$, and since $\tau^{0}\left(\mathbf{1}_{\mathcal{M}^{0}}\right)=1=\left\|\tau^{0}\right\|, \tau^{0}$ is a state on $\mathcal{M}^{0}$.

Consider next the GNS-representation $\pi_{\tau^{0}}: \mathcal{M}^{0} \rightarrow \mathcal{B}\left(\mathcal{H}_{\tau^{0}}\right)$ of $\mathcal{M}^{0}$ associated with $\tau^{0}$ (see [9, Theorem 4.5.2]), and let $\xi_{0}$ denote the unit $\mathbf{1}_{\mathcal{M}^{0}}$ of $\mathcal{M}^{0}$ considered as an element 
of $\mathcal{H}_{\tau_{0}}$. Let $\mathcal{M}$ denote the closure of $\pi_{\tau^{0}}\left(\mathcal{M}^{0}\right)$ in the strong operator topology, and define $\tau: \mathcal{M} \rightarrow \mathbb{C}$ by

$$
\tau(a)=\left\langle a \xi_{0}, \xi_{0}\right\rangle, \quad(a \in \mathcal{M}) .
$$

Then $\tau^{0}=\tau \circ \pi_{\tau^{0}}$, so $\tau$ is tracial on $\pi_{\tau^{0}}\left(\mathcal{N}^{0}\right)$. Since multiplication is separately continuous in each variable in the strong operator topology, it follows by a "bootstrap" argument that $\tau$ is tracial on all of $\mathcal{M}$. Hence $\xi_{0}$ is a generating trace vector for $\mathcal{M}$ and hence also for the commutant $\mathcal{M}^{\prime}$ (see [10, Lemma 7.2.14]). This implies that $\xi_{0}$ is separating for $\mathcal{M}$ (see [9, Corollary 5.5.12]), and hence $\tau$ is faithful, so that $(\mathcal{M}, \tau)$ is indeed a $W^{*}$-probability space.

For any $S$ in $\mathcal{S}$ we define next $\Phi_{S}: \mathcal{M}_{S} \rightarrow \mathcal{M}$ by $\Phi_{S}=\pi_{\tau^{0}} \circ \Phi_{S}^{0}$, and we note for $a$ in $\mathcal{M}_{S}$ that

$$
\tau \circ \Phi_{S}(a)=\left(\tau \circ \pi_{\tau^{0}}\right) \circ \Phi_{S}^{0}(a)=\tau^{0} \circ \Phi_{S}^{0}(a)=\tau^{00} \circ \Phi_{S}^{0}(a)=\tau_{S}(a) .
$$

Hence Corollary A.2 implies that $\Phi_{S}$ is injective and normal. If $S, T \in \mathcal{S}$, such that $S \leq T$, and $a \in \mathcal{M}_{S}$, we note furthermore that

$$
\Phi_{T} \circ \Phi_{S, T}(a)=\pi_{\tau^{0}} \circ \Phi_{T}^{0} \circ \Phi_{S, T}=\pi_{\tau^{0}} \circ \Phi_{S}^{0}(a)=\Phi_{S}(a) .
$$

To see that $\mathcal{M}$ is generated as a von Neumann algebra by $\bigcup_{S \in \mathcal{S}} \Phi_{S}\left(\mathcal{M}_{S}\right)$ we use again that $\mathcal{M}^{0}=\left(\bigcup_{S \in \mathcal{S}} \Phi_{S}^{0}\left(\mathcal{M}_{S}\right)\right)=$ (where $\mathcal{C}=$ denotes the norm closure of $\mathcal{C}$ ). Since $\pi_{\tau^{0}}$ is norm-continuous and $\pi_{\tau^{0}}\left(\mathcal{M}^{0}\right)$ is a $C^{*}$-algebra, this implies that

$$
\pi_{\tau^{0}}\left(\mathcal{M}^{0}\right)=\left(\bigcup_{S \in \mathcal{S}} \pi_{\tau^{0}} \circ \Phi_{S}^{0}\left(\mathcal{M}_{S}\right)\right)=\left(\bigcup_{S \in \mathcal{S}} \Phi_{S}\left(\mathcal{M}_{S}\right)\right)^{=} .
$$

Since the norm topology is stronger than the strong operator topology, this further entails that

$$
\mathcal{M}=\left(\pi_{\tau^{0}}\left(\mathcal{M}^{0}\right)\right)^{-s}=\left(\left(\bigcup_{S \in \mathcal{S}} \Phi_{S}\left(\mathcal{M}_{S}\right)\right)^{=}\right)^{-s}=\left(\bigcup_{S \in \mathcal{S}} \Phi_{S}\left(\mathcal{M}_{S}\right)\right)^{-s}
$$

(where $\mathcal{C}^{-s}$ denotes the closure of $\mathcal{C}$ in the strong operator topology) as desired.

We establish finally the uniqueness statement. If $\left(\mathcal{M}^{\prime}, \tau^{\prime}\right)$ is another $W^{*}$-probability space satisfying the conditions listed for $(\mathcal{M}, \tau)$, we consider the injective *-monomorphisms $\Phi_{S}^{\prime}: \mathcal{M}_{S} \rightarrow \mathcal{M}^{\prime}$ corresponding to the $\Phi_{S}$ 's. It follows then that the two families of operators $\bigcup_{S \in \mathcal{S}} \Phi_{S}\left(\mathcal{M}_{S}\right)$ and $\bigcup_{S \in \mathcal{S}} \Phi_{S}^{\prime}\left(\mathcal{M}_{S}\right)$ (indexed by $\left.\bigcup_{S \in \mathcal{S}} \mathcal{M}_{S}\right)$ have the same $*$-distribution. Hence Proposition A.1 yields an injective, normal $*$-isomorphism $\Psi$ from $\mathcal{M}=\left(\bigcup_{S \in \mathcal{S}} \Phi_{S}\left(\mathcal{M}_{S}\right)\right)^{-s}$ onto $\left(\bigcup_{S \in \mathcal{S}} \Phi_{S}^{\prime}\left(\mathcal{M}_{S}\right)\right)^{-s}=\mathcal{M}^{\prime}$, such that $\tau=\tau^{\prime} \circ \Psi$.

Before stating the next proposition we recall that the symbol “ $\stackrel{P}{\rightarrow}$ ” refers to convergence in the measure topology.

Proposition A.4. Let $(\mathcal{N}, \tau)$ and $(\mathcal{N}, \psi)$ be $W^{*}$-probability spaces, and let $\Phi: \mathcal{M} \rightarrow \mathcal{N}$ be a $*$-homomorphism such that $\tau=\psi \circ \Phi$. Let further $\overline{\mathcal{M}}$ and $\overline{\mathcal{N}}$ denote the set of (closed densely defined) operators affiliated with $\mathcal{M}$ and $\mathcal{N}$, respectively. We then have

(i) $\Phi$ extends to an injective mapping $\bar{\Phi}: \overline{\mathcal{M}} \rightarrow \overline{\mathcal{N}}$ which preserves the operations of scalar multiplication, strong sum, strong multiplication and the *-operation. In addition $\bar{\Phi}(\overline{\mathcal{M}})=\overline{\Phi(\mathcal{M})}$, and $(\bar{\Phi})^{-1}=\overline{\Phi^{-1}}$.

(ii) If $\left(a_{l}\right)_{l \in \mathbb{N}}$ is a sequence of operators from $\mathcal{M}, a \in \overline{\mathcal{M}}$ and $a_{l} \stackrel{\mathrm{P}}{\rightarrow} a$, then also $\Phi\left(a_{l}\right) \stackrel{P}{\rightarrow} \bar{\Phi}(a)$.

(iii) $\bar{\Phi}$ preserves spectral calculus in the sense that

$$
\bar{\Phi}(f(a))=f(\bar{\Phi}(a))
$$

for any selfadjoint operator $a$ in $\overline{\mathcal{M}}$ and any function $f$ from $\mathcal{B F}(\mathbb{R})$. 
Proof. Since $\tau$ is a finite trace it follows from [18, Example 1, page 22] that $\overline{\mathcal{M}}$ equals the class of $\tau$-measurable operators affiliated with $\mathcal{M}$. Hence (see [18, Theorem 28]) $\overline{\mathcal{M}}$ is a complete Hausdorff topological $*$-algebra with respect to the measure topology. In addition $\mathcal{M}$ is dense in $\overline{\mathcal{M}}$ with respect to the measure topology, and this topology is first countable. Of course similar statements hold for $\overline{\mathcal{N}}$ in relation to $(\mathcal{N}, \psi)$.

Now given $a$ in $\overline{\mathcal{M}}$ we may choose a sequence $\left(a_{n}\right)_{n \in \mathbb{N}}$ from $\mathcal{M}$ such that $a_{n} \stackrel{\mathrm{P}}{\rightarrow} a$. Since $\Phi$ is normal it follows then for any positive $\epsilon$ that

$$
\begin{aligned}
\psi\left[1_{[\epsilon, \infty)}\left(\left|\Phi\left(a_{n}\right)-\Phi\left(a_{m}\right)\right|\right)\right] & =\psi\left[1_{[\epsilon, \infty)}\left(\left|\Phi\left(a_{n}-a_{m}\right)\right|\right)\right]=\psi\left[1_{[\epsilon, \infty)}\left(\Phi\left(\left|a_{n}-a_{m}\right|\right)\right)\right] \\
& =\psi\left[\Phi\left(1_{[\epsilon, \infty)}\left(\left|a_{n}-a_{m}\right|\right)\right)\right]=\tau\left[1_{[\epsilon, \infty)}\left(\left|a_{n}-a_{m}\right|\right)\right] \longrightarrow 0,
\end{aligned}
$$

as $n, m \rightarrow \infty$. Hence $\left(\Phi\left(a_{n}\right)\right)_{n \in \mathbb{N}}$ is a Cauchy sequence in $\mathcal{N}(\subseteq \overline{\mathcal{N}})$ with respect to the measure topology, so there exists an element $b$ in $\overline{\mathcal{N}}$ such that $\Phi\left(a_{n}\right) \stackrel{\mathrm{P}}{\rightarrow} b$ as $n \rightarrow \infty$. Considering another sequence $\left(a_{n}^{\prime}\right)_{n \in \mathbb{N}}$ from $\mathcal{M}$ such that $a_{n}^{\prime} \stackrel{P}{\rightarrow} a$, we may further consider the mixed sequence $a_{1}, a_{1}^{\prime}, a_{2}, a_{2}^{\prime}, \ldots$ which also converges to $a$ in probability. Hence the argument above shows that the sequences $\left(\Phi\left(a_{n}^{\prime}\right)\right)_{n \in \mathbb{N}}$ and $\Phi\left(a_{1}\right), \Phi\left(a_{1}^{\prime}\right), \Phi\left(a_{2}\right), \Phi\left(a_{2}^{\prime}\right), \ldots$ converge in probability to elements $b^{\prime}$ respectively $b^{\prime \prime}$ from $\overline{\mathcal{N}}$. By subsequence considerations we must have that $b=b^{\prime \prime}=b^{\prime}$, and hence we may define a mapping $\bar{\Phi}: \overline{\mathcal{M}} \rightarrow \overline{\mathcal{N}}$ by setting

$$
\bar{\Phi}(a)=\mathrm{P}-\lim _{n \rightarrow \infty} \Phi\left(a_{n}\right), \quad(a \in \overline{\mathcal{M}}),
$$

where $\left(a_{n}\right)_{n \in \mathbb{N}}$ is any sequence from $\mathcal{M}$ such that $a_{n} \stackrel{\mathrm{P}}{\rightarrow} a$ as $n \rightarrow \infty$.

From this definition and the fact that scalar multiplication, strong sum, strong multiplication and the $*$-operation are all continuous operations in the measure topology, it follows by standard arguments that

$$
\bar{\Phi}(\lambda a)=\lambda \Phi(a), \quad \bar{\Phi}(a+b)=\bar{\Phi}(a)+\bar{\Phi}(b), \quad \bar{\Phi}(a b)=\bar{\Phi}(a) \bar{\Phi}(b), \quad \bar{\Phi}\left(a^{*}\right)=\bar{\Phi}(a)^{*}
$$

for any $a, b$ from $\overline{\mathcal{M}}$ and $\lambda$ in $\mathbb{C}$. In other words $\bar{\Phi}$ is a $*$-homomorphism.

Recalling from Lemma A.2 that $\Phi(\mathcal{M})$ is a von Neumann subalgebra of $\mathcal{N}$, we check next that $\bar{\Phi}(\overline{\mathcal{N}})=\overline{\Phi(\mathcal{M})}$. Since $\overline{\Phi(\mathcal{M})}$ is the closure of $\Phi(\mathcal{M})$ in the measure topology, the definition (A.3) clearly implies that $\bar{\Phi}(\overline{\mathcal{M}}) \subseteq \overline{\Phi(\mathcal{M})}$. Conversely, given $b$ in $\overline{\Phi(\mathcal{M})}$, we may choose a sequence $\left(a_{n}\right)_{n \in \mathbb{N}}$ from $\mathcal{M}$ such that $\Phi\left(a_{n}\right) \stackrel{\text { P }}{\rightarrow} b$. The calculation (A.2) then shows that $\left(a_{n}\right)_{n \in \mathbb{N}}$ is a Cauchy sequence and hence convergent in the measure topology to some $a$ from $\overline{\mathcal{M}}$. Now (A.3) implies that $b=\bar{\Phi}(a) \in \bar{\Phi}(\overline{\mathcal{M}})$. The mapping $\Phi^{-1}: \Phi(\mathcal{M}) \rightarrow \mathcal{M}$ (cf. Corollary A.2) similarly gives rise to a mapping $\overline{\Phi^{-1}}: \overline{\Phi(\mathcal{M})} \rightarrow \overline{\mathcal{M}}$, and it follows easily from (A.3) (and the corresponding definition of $\overline{\Phi^{-1}}$ ) that $\overline{\Phi^{-1}} \circ \bar{\Phi}(a)=a$ for all $a$ in $\overline{\mathcal{M}}$ and that $\bar{\Phi} \circ \overline{\Phi^{-1}}(b)=b$ for all $b$ in $\overline{\Phi(\mathcal{N})}$. In particular $\bar{\Phi}$ is injective.

Consider finally a selfadjoint element $a$ from $\overline{\mathcal{M}}$, put $b=\bar{\Phi}(a) \in \overline{\mathcal{N}}$ and note that $b=b^{*}$. We then define a mapping $\Psi: \mathcal{B F}(\mathbb{R}) \rightarrow \overline{\mathcal{N}}$ by setting

$$
\Psi(f)=\bar{\Phi}(f(a)), \quad(f \in \mathcal{B} \mathcal{F}(\mathbb{R})) .
$$

We show next that $\Psi(f) \in \overline{\Phi\left(W^{*}(\{a\})\right)}$ for all $f$ in $\mathcal{B F}(\mathbb{R})$, where $W^{*}(\{a\})$ denotes the (abelian) von Neumann sub-algebra of $\mathcal{M}$ generated by $a$. For this note first that $\Phi\left(W^{*}(\{a\})\right)$ is again a von Neumann algebra (cf. Lemma A.2). Given $f$ in $\mathcal{B F}(\mathbb{R})$ we put $f_{n}=f 1_{\{|f| \leq n\}}$ and note that $f_{n}(a) \in W^{*}(\{a\})$ for all $n$. Using [9, Corollary 5.6.29] we find then that

$$
\begin{aligned}
\tau\left[1_{[\epsilon, \infty)}\left(\left|f_{n}(a)-f(a)\right|\right)\right] & =\tau\left[\left(1_{[\epsilon, \infty)} \circ\left|f_{n}-f\right|\right)(a)\right]=\int_{\mathbb{R}} 1_{\left\{\left|f_{n}-f\right| \geq \epsilon\right\}}(t) L^{\mathrm{sp}}\{a\}(\mathrm{d} t) \\
& =L^{\mathrm{sp}}(\{a\})\left(\left\{\left|f_{n}-f\right| \geq \epsilon\right\}\right) \underset{n \rightarrow \infty}{\longrightarrow} 0,
\end{aligned}
$$


where we used that $f_{n} \rightarrow f$ point-wise and that $L^{\mathrm{sp}}\{a\}$ is a finite measure. Thus $f_{n}(a) \stackrel{\mathrm{P}}{\rightarrow} f(a)$ and hence also $\Phi\left(f_{n}(a)\right) \stackrel{\mathrm{P}}{\rightarrow} \bar{\Phi}(f(a))$. Since $\overline{\Phi\left(W^{*}(\{a\})\right)}$ is complete in the measure topology, it follows that $\bar{\Phi}(f(a)) \in \overline{\Phi\left(W^{*}(\{a\})\right)}$ as desired, and in particular we have that $b=\bar{\Phi}(a) \in \overline{\Phi\left(W^{*}(\{a\})\right)}$.

Note next that $\Psi$ is a $*$-homomorphism (since both $\bar{\Phi}$ and the mapping $f \mapsto f(a)$ are *-homomorphisms), and furthermore $\Psi$ is $\sigma$-normal in the sense of $[9,10]$, since the mapping $f \mapsto f(a)$ is $\sigma$-normal (cf. [9, Theorem 5.6.26]), and since $\bar{\Phi}$ preserves least upper bounds (because $\bar{\Phi}$ and $\bar{\Phi}^{-1}$ both preserve positivity).

The observations above allow us to apply [9, Theorem 5.6.27] by which we infer that $\Psi$ is the spectral mapping associated to $b$, i.e. $f(\bar{\Phi}(a))=\bar{\Phi}(f(a))$ for all $f$ in $\mathcal{B} \mathcal{F}(\mathbb{R})$. This completes the proof.

Corollary A.5. Let $(\mathcal{N}, \tau),(\mathcal{N}, \psi)$ and $(\mathcal{L}, \varpi)$ be $W^{*}$-probability spaces, and let $\Phi: \mathcal{M} \rightarrow \mathcal{N}$ and $\Gamma: \mathcal{N} \rightarrow \mathcal{L}$ be $*$-homomorphisms such that $\tau=\psi \circ \Phi$, and $\psi=\varpi \circ \Gamma$.

Then $\Phi$ and $\Gamma$ are both normal and injective, and for any $a$ in $\overline{\mathcal{M}}$ we have that $\overline{\Gamma \circ \Phi}(a)=\bar{\Gamma} \circ \bar{\Phi}(a)$.

Proof. Lemma A.2 ensures that $\Phi, \Gamma$ and $\Gamma \circ \Phi$ are normal and injective, and Proposition A.4 ensures that the mappings $\bar{\Phi}, \bar{\Gamma}$ and $\overline{\Gamma \circ \Phi}$ are all well-defined.

Given $a$ in $\overline{\mathcal{M}}$ we choose a sequence $\left(a_{n}\right)_{n \in \mathbb{N}}$ from $\mathcal{M}$ converging to $a$ in the measure topology. Proposition A.4 then entails that $\Phi\left(a_{n}\right) \stackrel{\mathrm{P}}{\rightarrow} \bar{\Phi}(a)$, and hence that $\Gamma \circ \Phi\left(a_{n}\right) \stackrel{\mathrm{P}}{\rightarrow}$ $\bar{\Gamma}(\bar{\Phi}(a))$. In addition $\Gamma \circ \Phi\left(a_{n}\right) \stackrel{\mathrm{P}}{\rightarrow} \overline{\Gamma \circ \Phi}(a)$, and since the measure topology is a Hausdorff topology, we obtain the desired conclusion.

\section{References}

[1] O.E. Barndorff-Nielsen and S. Thorbjørnsen: A connection between free and classical infinite divisibility. Infin. Dimens. Anal. Quantum Probab. Relat. Top. 7(4) (2004), 573-590. MR2105912

[2] O.E. Barndorff-Nielsen and S. Thorbjørnsen: The Lévy-Itô decomposition in free probability. Probab. Theory Related Fields 131(2) (2005), 197-228. MR-2117952

[3] O.E. Barndorff-Nielsen and S. Thorbjørnsen: Classical and free infinite divisibility and Lévy processes. In U. Franz and M. Schürmann (Eds.): Quantum independent increment processes II. Quantum Lévy processes, classical probability and applications to physics, 33-159, Springer, Berlin (2006). MR-2213448

[4] O.E. Barndorff-Nielsen, J. Rosiński, S. Thorbjørnsen: General ؟-transformations. ALEA Lat. Am. J. Probab. Math. Stat. 4 (2008), 131-165. MR-2421179

[5] F. Benaych-Georges: Taylor expansions of $R$-transforms: application to supports and moments. Indiana Univ. Math. J. 55(2) (2006), 465-481. MR-2225442

[6] H. Bercovici and V. Pata: A Free Analogue of Hinčin's characterization of infinite divisibility. Proc. Amer. Math. Soc. 128(4) (2000), 1011-1015. MR-1636930

[7] H. Bercovici and D.V. Voiculescu: Free convolution of measures with unbounded support. Indiana Univ. Math. J. 42(3) (1993), 733-773. MR-1254116

[8] J.A. Domínguez-Molina and A. Rocha-Arteaga: Random matrix models of stochastic integral type for freely infinitely divisible distributions. Period. Math. Hungar. 64(2) (2012), 145-160. MR-2925165

[9] R.V. Kadison and J.R. Ringrose: Fundamentals of the theory of operator algebras, vol I. Elementary theory. Graduate Studies in Mathematics, 15. American Mathematical Society, Providence (1997). MR-1468229

[10] R.V. Kadison and J.R. Ringrose: Fundamentals of the theory of operator algebras, vol II. Advanced theory. Graduate Studies in Mathematics, 15. American Mathematical Society, Providence (1997). MR-1468230 
Completely random measures and Lévy bases in free probability

[11] J.F.C. Kingman: Completely random measures. Pacific. J. Math. 21 (1967), 59-78. MR-210185

[12] J. Mingo and R. Speicher: Free probability and random matrices. Fields Institute Monographs, 35. Springer, New York; Fields Institute for Research in Mathematical Sciences, Toronto (2017). MR-3585560

[13] J.C. Pardo, J.L. Pérez and V. Pérez-Abreu: On the non-commutative fractional Wishart process J. Funct. Anal. 272(1) (2017), 339-362. MR-3567507

[14] V. Pérez-Abreu and A. Rocha-Arteaga: On the process of the eigenvalues of a Hermitian Lévy process. In The fascination of probability, statistics and their applications, 231-249, Springer, Cham (2016). MR-3495687

[15] J. Pedersen: The Lévy-Ito decomposition of an independently scattered random measure. MaPhySto Research Report 2 (2003), University of Aarhus.

[16] B.S Rajput and J. Rosiński: Spectral representations of infinitely divisble processes. Probab. Theory Related Fields 82(3) (1989), 451-487. MR-1001524

[17] K. Sato: Lévy processes and infinitely divisible distributions. Cambridge Studies in Advanced Mathematics, 68. Cambridge University Press, Cambridge (1999). MR-1739520

[18] M. Terp: $L^{p}$ Spaces associated with von Neumann Algebras. Lecture notes, University of Copenhagen (1981).

[19] K. Urbanik and W.A. Woyczyński: A random integral and Orlicz spaces. Bull. Acad. Polon. Sci. Sér. Sci. Math. Astronom. Phys. 15 (1967), 161-169. MR-215329

[20] D.V. Voiculescu: Circular and semicircular systems and free product factors. Operator algebras, unitary representations, enveloping algebras and invariant theory, 45-60, Progr. Math., 92. Birkhäuser Boston, Boston (1990). MR-1103585

[21] D.V. Voiculescu, K.J. Dykema and A. Nica: Free Random Variables. A noncommutative probability approach to free products with applications to random matrices, operator algebras and harmonic analysis on free groups. CRM Monograph Series, 1. American Mathematical Society, Providence (1992). MR-1217253 


\section{Electronic Journal of Probability Electronic Communications in Probability}

\section{Advantages of publishing in EJP-ECP}

- Very high standards

- Free for authors, free for readers

- Quick publication (no backlog)

- Secure publication $\left(\mathrm{LOCKSS}^{1}\right)$

- Easy interface (EJMS²)

\section{Economical model of EJP-ECP}

- Non profit, sponsored by $\mathrm{IMS}^{3}, \mathrm{BS}^{4}$, ProjectEuclid ${ }^{5}$

- Purely electronic

\section{Help keep the journal free and vigorous}

- Donate to the IMS open access fund ${ }^{6}$ (click here to donate!)

- Submit your best articles to EJP-ECP

- Choose EJP-ECP over for-profit journals

\footnotetext{
${ }^{1}$ LOCKSS: Lots of Copies Keep Stuff Safe http://www. lockss.org/

${ }^{2}$ EJMS: Electronic Journal Management System http://www.vtex.lt/en/ejms.html

${ }^{3}$ IMS: Institute of Mathematical Statistics http://www.imstat.org/

${ }^{4}$ BS: Bernoulli Society http://www. bernoulli-society.org/

${ }^{5}$ Project Euclid: https://projecteuclid.org/

${ }^{6}$ IMS Open Access Fund: http://www.imstat.org/publications/open.htm
} 